NIST Technical Note 2147

\title{
Characterizing LTE User Equipment Emissions Under Closed-Loop Power Control
}

\author{
Jason Coder \\ Aric Sanders \\ Michael Frey \\ Adam Wunderlich \\ Azizollah Kord \\ Jolene Splett \\ Lucas Koepke \\ Daniel Kuester \\ Duncan McGillivray \\ John Ladbury
}

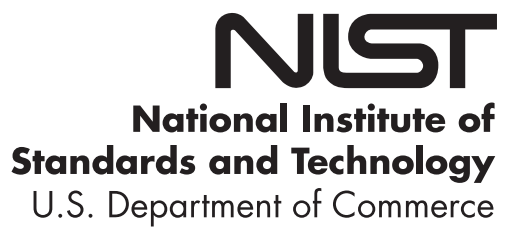


NIST Technical Note 2147

\title{
Characterizing LTE User Equipment Emissions Under Closed-Loop Power Control
}

\author{
Jason Coder \\ Aric Sanders \\ Adam Wunderlich \\ Azizollah Kord \\ Daniel Kuester \\ Duncan McGillivray \\ John Ladbury \\ Communications Technology Laboratory \\ NIST \\ Michael Frey \\ Jolene Splett \\ Lucas Koepke \\ Information Technology Laboratory
}

NIST

This publication is available free of charge from: https://doi.org/10.6028/NIST.TN.2147

April 2021

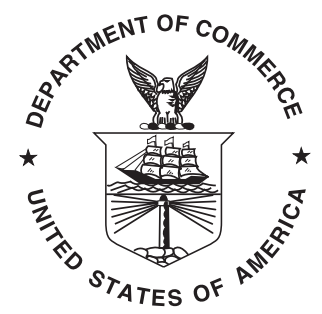

U.S. Department of Commerce Gina M. Raimondo, Secretary

National Institute of Standards and Technology James K. Olthoff, Performing the Non-Exclusive Functions and Duties of the Under Secretary of Commerce for Standards and Technology \& Director, National Institute of Standards and Technology 


\section{Characterizing LTE User Equipment Emissions Under Closed-Loop Power Control}

Jason Coder, Aric Sanders, Michael Frey, Adam Wunderlich, Azizollah Kord, Jolene Splett, Lucas Koepke, Daniel Kuester, Duncan McGillivray, John Ladbury

NASCTN Project Manager: Matthew Briel NASCTN Program Manager: Melissa Midzor

April 6, 2021
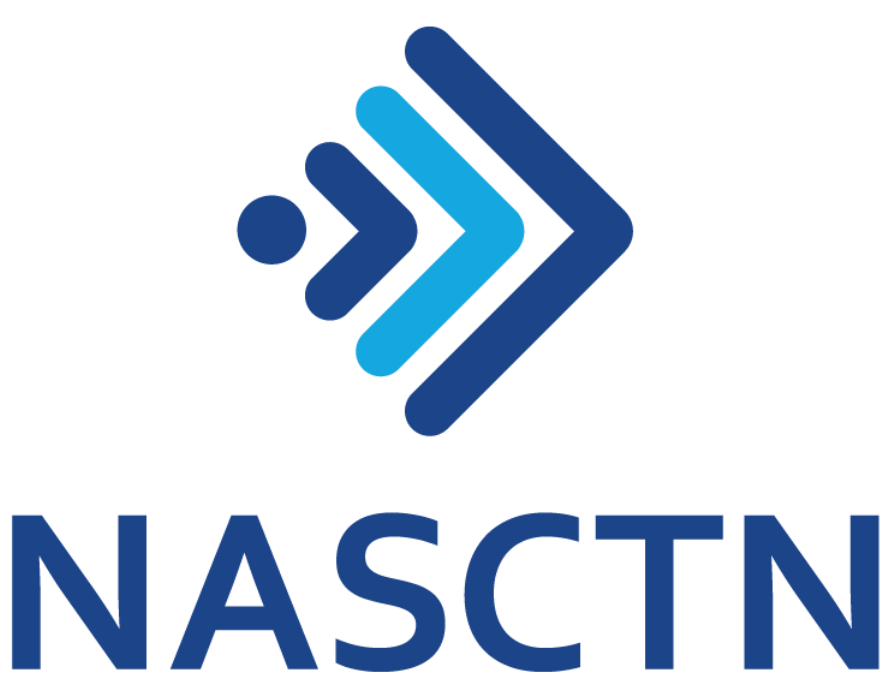

National Advanced Spectrum and Communications Test Network 


\section{DISCLAIMER}

Certain commercial entities, equipment, or materials may be identified in this document in order to describe an experimental procedure or concept adequately. Such identification does not imply any recommendation or endorsement by National Institute of Standards and Technology (NIST) or National Advanced Spectrum and Communications Test Network (NASCTN), nor is it intended to imply that the materials or equipment identified are necessarily the best available for the purpose.

National Institute of Standards and Technology Technical Note 2147

Natl. Inst. Stand. Technol. Tech. Note 2147, 104 pages (April 2021)

CODEN: NTNOEF

This publication is available free of charge from: https://doi.org/10.6028/NIST.TN.2147 


\section{Preface}

The work described herein was performed as a result of a spectrum sharing project request submitted to the National Advanced Spectrum and Communications Test Network (NASCTN). NIST is a founding charter member of NASCTN. This work was sponsored by Department of Defense (DoD) Defense Spectrum Organization (DSO), and conducted by NIST with support by MITRE. A summary of each organization's technical contributions follows.

The project underwent formal screening and approval by the NASCTN Steering Committee. A description of NASCTN and listing of Charter Members at the time of publication of this report follows. 


\section{National Advanced Spectrum and Communications Test Network (NASCTN)}

The mission of the National Advanced Spectrum and Communications Test Network (NASCTN) is to provide, through its members, a network for robust test processes and validated measurement data necessary to develop, evaluate and deploy spectrum sharing technologies that can improve access to the spectrum by both federal agencies and non-federal spectrum users.

NASCTN is a member organization under a charter agreement. Members

- Facilitate and coordinate work with federal, academic, and industry spectrum users to rapidly and cooperatively facilitate spectrum sharing and co-existence studies;

- Work as a partnership to address the interests and equities of all spectrum stakeholders in a fair, equitable, and non-preferential manner; and

- Through sharing of technical resources, with consideration for cost, provide liaison and support to coordinate and leverage existing national capabilities supporting government, academic, and industry testing and evaluation known to improve and expedite spectrum sharing and coexistence.

Charter members at the time of publication of this report are (in alphabetical order):

- Department of Defense Chief Information Officer (DoD CIO)

- National Aeronautics and Space Administration (NASA)

- National Institute of Standards and Technology (NIST)

- National Oceanic and Atmospheric Administration (NOAA)

- National Science Foundation (NSF)

- National Telecommunications and Information Administration (NTIA)

NIST hosts the NASCTN capability at the Department of Commerce Boulder Laboratories in Boulder, Colorado. 


\section{Technical Contributors}

\begin{tabular}{lll} 
Name & Organization & Primary Contribution Areas \\
\hline Jason Coder & NIST & Technical lead; testbed development and test execution \\
Aric Sanders & NIST (Assoc.) & Testbed automation, data processing, \& engineering data analysis \\
Michael Frey & NIST & Statistical analysis lead \\
Adam Wunderlich & NIST & Experimental design \& engineering data analysis \\
Aziz Kord & NIST & LTE engineering \\
Jolene Splett & NIST & Statistical analysis \\
Lucas Koepke & NIST & Statistical analysis \\
Daniel Kuester & NIST & Testbed automation \\
Duncan McGillivray & NIST & RF testbed design \& calibration \\
John Ladbury & NIST & RF uncertainty analysis
\end{tabular}




\section{Acknowledgments}

The authors express their thanks to the Defense Spectrum Organization (DSO) for the trust and confidence they have placed in National Advanced Spectrum and Communications Test Network (NASCTN) to conduct these measurements and document the results that will be used by multiple organizations conducting research on 3rd generation partnership's long term evolution protocols.

The authors also thank the National Institute of Standards and Radio Frequency Technology Division for their support in this measurement effort for NASCTN. Doing so required the support of former Division Chief Dr. Michael Janezic, and current Division Chief Dr. Paul Hale. The successful execution of this measurement campaign required a diverse set of skills from multiple organizations. MITRE Corporation personnel provided key project support in several areas. Namely, LTE expertise and assistance was provided by Jeffrey Corriea, Venkatesh Ramaswamy, Jerediah Fevold, and Shawn Lefebre. Mark Lofquist, formerly with the MITRE corporation, also aided the project by serving as a valuable liaison between the project and many of its DoD stakeholders.

Finally, we would like to thank the many additional individuals that made valuable contributions over the course of this project. Namely, we would like to acknowledge the efforts of the test plan development team and thank those who participated in the public comment period for the test plan. Also, the technical team thanks program staff from the NASCTN for their efforts, which made project success possible. Namely, we thank current NASCTN program manager, Dr. Melissa Midzor, former NASCTN project managers Dr. Keith Hartley \& Dr. Fabio da Silva, former NASCTN administrative officer, Linda Derr, and current NASCTN administrative officer Amanda Hyman. 


\section{Executive Summary}

In 2014, the Federal Communications Commission (FCC) (Order FCC 14-31) established the advanced wireless services 3 (AWS-3) Band: $1695 \mathrm{MHz}$ to $1710 \mathrm{MHz}, 1755 \mathrm{MHz}$ to $1780 \mathrm{MHz}$, and $2155 \mathrm{MHz}$ to $2180 \mathrm{MHz}$. The FCC auctioned the AWS-3 band for commercial mobile broadband usage in the United States, which raised $\$ 41 \mathrm{~B}$ and required federal agencies in the AWS-3 band to look for other ways to accomplish their missions outside of the AWS-3 band. In $1755 \mathrm{MHz}$ to $1780 \mathrm{MHz}$, the Department of Defense (DoD) is using a combination of sharing, consolidation, and relocation to other bands. Requests from auction winners to enter the band before the DoD has transitioned out are evaluated through the DoD early entry portal analysis capability (EEPAC), managed by the Defense Information Systems Agency (DISA) Defense Spectrum Organization (DSO). Coordination for early entry into this band depends on an interference model that was developed and agreed to by the FCC, National Telecommunications and Information Administration (NTIA), DoD, and commercial interests.

The DISA DSO through the Spectrum Sharing Test and Demonstration (SSTD) program proposed to the National Advanced Spectrum and Communications Test Network (NASCTN) a measurement campaign to quantitatively characterize long-term evolution (LTE) uplink waveforms generated by user equipment (UE) in the $1755 \mathrm{MHz}$ to $1780 \mathrm{MHz}$ band with the intent to make realistic measurements of UE emissions. These measurements will be used for assessing interference to DoD systems that, for a time, will remain in the $1755 \mathrm{MHz}$ to $1780 \mathrm{MHz}$ band. A secondary goal of this characterization, with a documented methodology and uncertainty, is to give AWS-3 stakeholders more confidence in the coordination request evaluation (CRE) process.

The key factors impacting the uplink emissions of LTE UEs were experimentally identified and discussed in a 2019 report [1]. In that report, NASCTN outlined a suitable measurement methodology, uncertainty analysis, and a statistical analysis approach capable of investigating the cumulative and complex interactions of UE emissions and network response. This initial work utilized a statistical "factor screening" approach. This approach was powerful, and enabled the efficient identification of factors impacting UE uplink emissions. The final part of this earlier work involved detailing 14 additional questions which, if answered, would provide valuable information to the stakeholder community. These questions are briefly outlined at the end of [1].

After consultation with DISA DSO and the stakeholder community, NASCTN developed the final measurement test to further characterize the factors impacting UE uplink emissions when closed-loop power control is enabled in the cell, and modeled the emissions over operational scenarios. Closed-loop power control is a mechanism whereby the eNB actively gives feedback to UEs in its cell. If certain metrics fall under a given threshold, the eNB will send a command to the individual UE telling it to increase its transmit power. Similarly, when certain metrics exceed a given threshold, the eNB may issue a command to reduce UE transmit power. The goal of this mechanism is to optimize the power emitted by the UE. This is thought to improve battery life on portable devices, and prevent unnecessary emissions that could impact other devices in the cell. Closed-loop power control is an option on many eNBs; its use is not required by industry standards or regulators. The prevalence of closed-loop power control in real-world deployments is unknown.

Key products of this study include:

- Distributions of effective isotropic radiated power (EIRP) from a UE in an active resource block for a variety of test configurations (e.g., path loss, UE settings, and LTE network settings).

- Statistical models that describe the relationship between a subset of factors and the UE's uplink emissions.

- An exploratory data analysis that utilizes the acquired data to compare the UE's self-reported transmit power to measured distributions of EIRP and examines eNB scheduling dynamics in negative power headroom situations.

- Uncertainty analysis that includes an uncertainty budget and calculation of overall measurement uncertainty. 
- Summary statistics for the test data, publicly available at https://doi.org/10.18434/mds2-2358.

The objective of this test is to demonstrate the design of an experiment that characterizes the factors impacting UE uplink emissions while leveraging the test methodology, acquisition/automation software, and physical hardware setup developed in the "factor screening" phase of this work. The output of this work can aid in the development of emissions models and interference calculations in the AWS-3 band and beyond.

The primary aim of the work presented here is to determine which factors have a significant impact on the distribution of physical uplink shared channel (PUSCH) power per physical resource block (PRB) under closed-loop power control conditions. This type of experiment is referred to as a characterization-type test in the experimental design literature. This experiment, which involved six factors, was designed to yield statistical models that describe the relationship between the six factors and the UE's uplink emissions. Note, these descriptive models are categorically different than predictive models.

Though the methods and results discussed here specifically relate to a particular frequency band of interest, the methods can be repeated in other frequency bands, for other types of equipment (e.g., narrow-band Internet of Things (IoT) type devices), and for other types of networks (e.g., small cell deployments).

\section{Key Challenges:}

- Identifying and limiting the test to six factors from the larger list of potential factors. While this is still a large number of factors for a characterization test, the established testbed and automation enabled this to be completed within the timeframe needed.

- Our modeling of the centiles of the PUSCH EIRP per PRB distribution reveals a changeline separating two distinct regimes - one regime where the relationship between PUSCH EIRP per PRB and path loss is linear, and another regime at high path loss where PUSCH EIRP per PRB exhibits a plateau. This changeline was unanticipated, and the design points chosen for the modeling experiment were not well-targeted for its modeling. We ultimately succeeded in constructing a descriptive two-piece hyperbolic paraboloid (HP) model using a novel homotopy method. A homotopy solves a hard problem by starting with an easy problem and then slowly deforming that easy problem to look more and more like the desired problem, updating the solution at each step. This approach allowed us - with the limited data collected - to side-step the convergence and instability problems encountered in our initial attempts to directly fit the HP model to the modeling data.

- One of the secondary goals in designing the closed-loop power control experiments is that we sought realistic ranges for the values of all six factors. For some factors, determining the realistic range was tractable (e.g., path-loss). For other factors we were able to find some data sets (e.g., offered load). However, for some factors (e.g., scheduling method), no reliable data could be found. In those case, settings were selected based on an engineering analysis.

\section{Key Findings:}

\section{A descriptive model for UE uplink emissions was created based on our measured data. (see Section 5.3)}

- In closed-loop power control, in the low crosstalk scenario, the median uplink power of the UE is described by a linear relationship with path loss between the UE and the eNB. This linear relationship abruptly changes when the UE begins to emit its maximum allowable power $(+23 \mathrm{dBm})$. Beyond this transition, the slope becomes slightly negative. This transition typically corresponds to the UE being in negative power headroom.

- In the low crosstalk scenario, changing the fractional power control in a closed loop power control situation does not affect the median UE uplink power. This differs from the generally held view that changing the fractional power 
level ("alpha") would have an impact. The interference model includes this factor and assumes that this value is known. Note: This conclusion is restricted to the model of UE tested.

- The introduction of crosstalk conditions as a factor (i.e., interference from outside or within a cell) exposed an additional level of complexity. In particular, the high crosstalk scenario resulted in large variability that exceeded the study's controlled factors, thus limiting our ability to create a descriptive model of that effect.

2. eNB Scheduling algorithms do not meaningfully impact uplink emitted power. (see Section 5.3.1)

- The uplink scheduling algorithms of channel unaware, channel aware, or interference aware do not meaningfully affect median uplink power from the UE in the low crosstalk scenario under closed-loop power control conditions. The selection of a particular algorithm does however affect the PRB allocation location dependent on the algorithm selected. In channel unaware cases the PRB allocations are uniform in distribution across the allocated channel. In both the channel aware and interference aware cases the UE is allotted PRBs asymmetrically based on the RF conditions.

3. The UE's measurements and other self-reported RF metrics may differ significantly from independent measurements. (See Section 6.3)

- The UE self-reported power varies from actual measured EIRP of the UE by $7 \mathrm{~dB}$ on average, with a standard deviation of $2 \mathrm{~dB}$.

- The downlink path loss estimate used in calculating the power control equation at the UE varies significantly from other independent measurements.

\section{The conclusions reached are independent of UE serial number. (See Section 5.2)}

- Based on the four serial numbers of the same UE model tested, the same statistical conclusions are reached by analyzing any of the four devices tested.

The experiment design, testbed, data processing, and statistical analysis successfully worked together to produce very high resolution results. The data and models can be used to improve DoD/DSO interference risk assessment, inform modeling LTE UE emissions which is a key component of the DoD Aggregate Interference Models, and increase DoD/DSO's confidence in allowing commercial LTE systems to deploy. In addition, a summary of the NASCTN results in terms of contributions to larger modeling efforts can be found in Appendix B.3. 


\section{Contents}

Technical Contributors $\ldots \ldots \ldots \ldots \ldots \ldots \ldots \ldots \ldots \ldots \ldots \ldots \ldots \ldots$

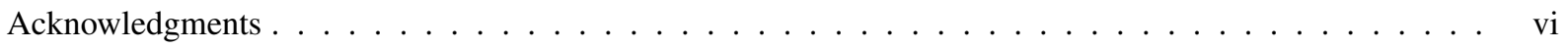

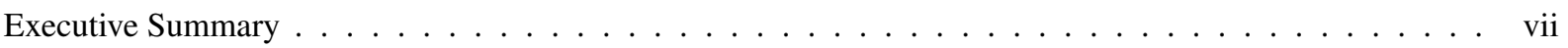

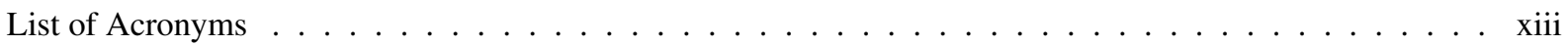

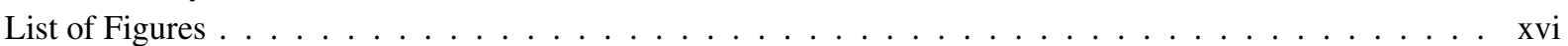

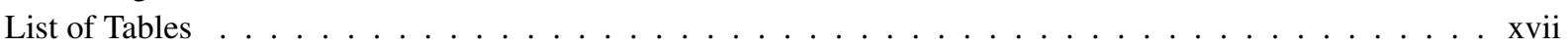

1 Introduction 1

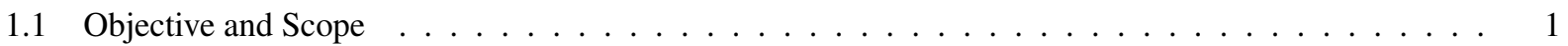

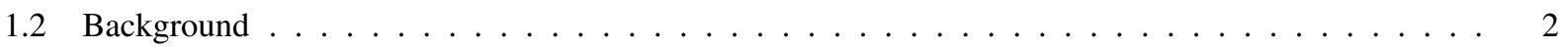

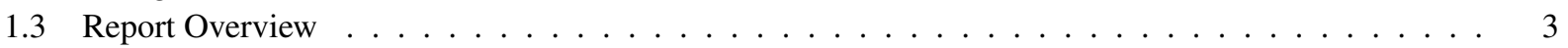

2 Experimental Factors $\quad 4$

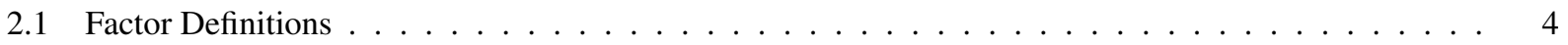

2.1.1 Factor A - Applied Attenuation/Path Loss . . . . . . . . . . . . . . . . . . . . 4

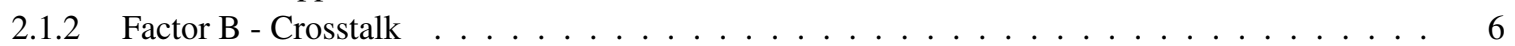

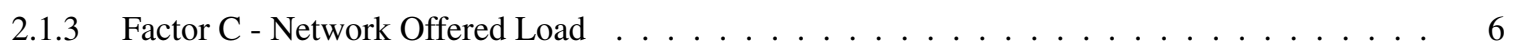

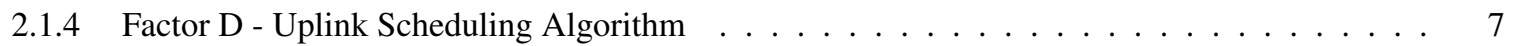

2.1.5 Factor E - Cell Specific Nominal Power Factor ～. . . . . . . . . . . . . . . . . . 7

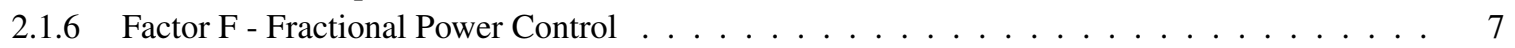

2.2 Collateral eNB Factors $\ldots \ldots \ldots \ldots \ldots \ldots$

3 Testbed Design $\quad 10$

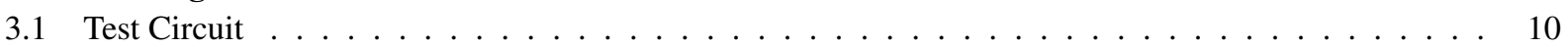

3.1 .1 UE Traffic Generator . . . . . . . . . . . . . . . . . . . . . . . . . . . . . . . 11

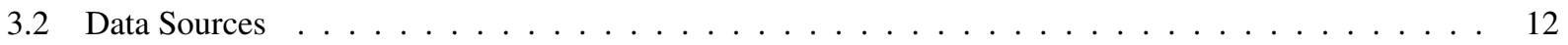

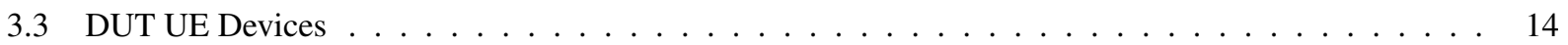

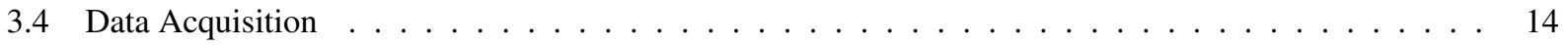

4 Experimental Design $\quad 15$

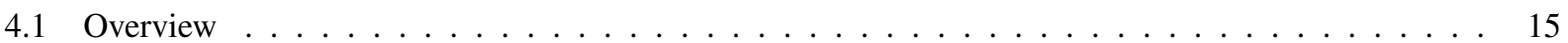

4.2 Design Details . . . . . . . . . . . . . . . . . . . . . . 16

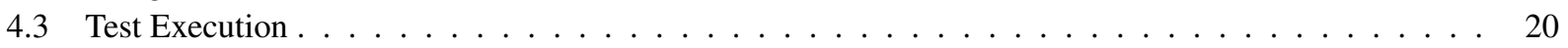

5 Statistical Analysis $\quad 21$

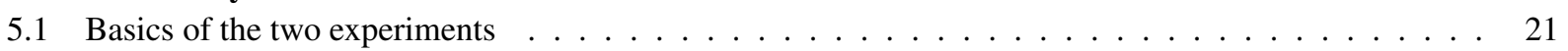

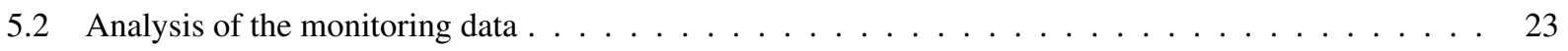

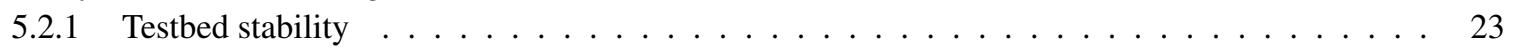

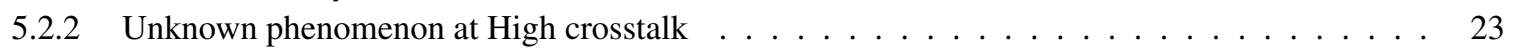

5.2 .3 Handset-contributed variability . . . . . . . . . . . . . . . . . . 27 


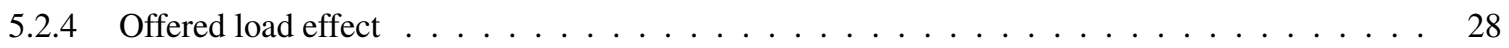

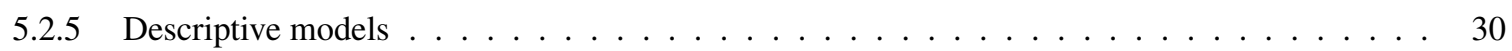

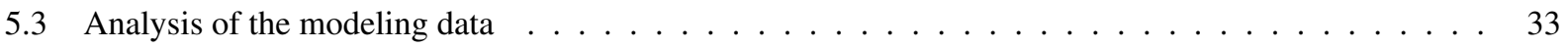

5.3.1 Effects of offered load, scheduling, and the power fraction $\ldots \ldots \ldots \ldots$

5.4 High crosstalk in the modeling experiment . . . . . . . . . . . . . . . . . . 41

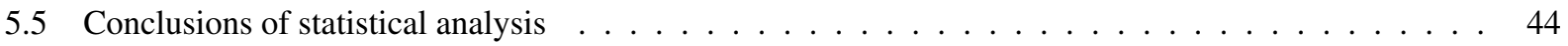

6 Engineering Analysis $\quad 45$

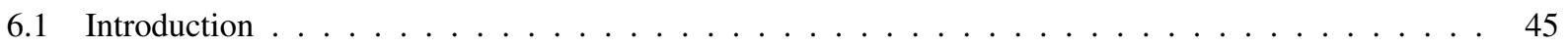

6.2 Experimental results in context of the power control equation $\ldots \ldots \ldots \ldots \ldots \ldots$

6.3 Comparison of measured EIRP and UE self-reported diagnostics (USRD) Power per PRB . . . . . . 50

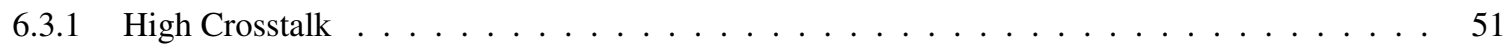

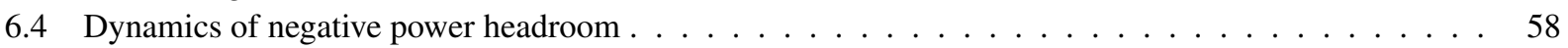

7 Conclusions \& Discussion $\quad 64$

7.1 Conclusions from statistical analysis $\ldots \ldots \ldots \ldots \ldots \ldots \ldots \ldots \ldots$

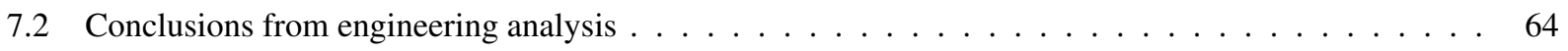

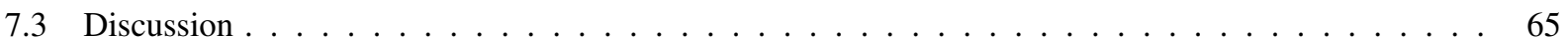

7.3.1 Applications to $5 \mathrm{G}$ New Radio . . . . . . . . . . . . . . . . . . . . 65

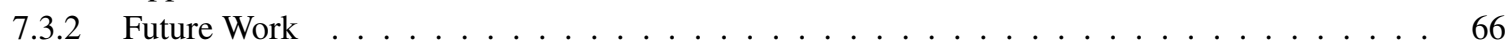

$\begin{array}{lll}\text { A Uncertainty Budget } & 67\end{array}$

A.1 Uncertainty . . . . . . . . . . . . . . . . . . . . . . . . 67

A.1.1 Vector Signal Analyzer Power Measurement Uncertainty Components . . . . . . . . . . . . 67

A.1.2 Coupled Output Power Calibration Uncertainty Components . . . . . . . . . . . . . . . . . 67

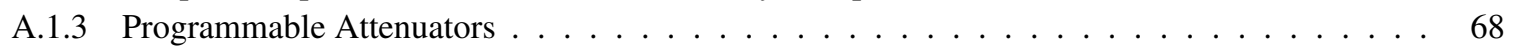

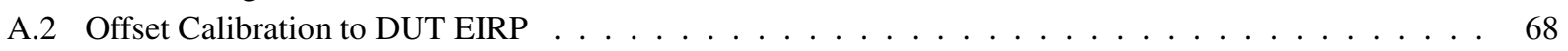

B Applications to Modeling: A Summary of

$\begin{array}{ll}\text { NASCTN Findings } & \mathbf{7 0}\end{array}$

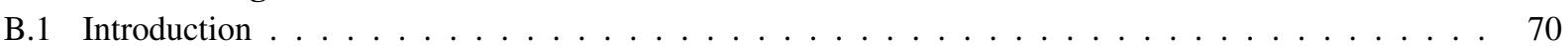

B.2 Descriptive, Explanatory, and Predictive Models . . . . . . . . . . . . . . . . . . 70

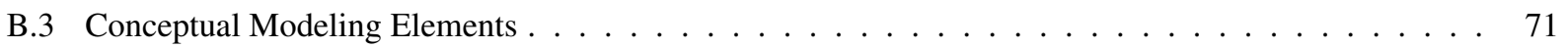

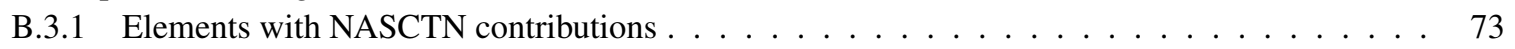

B.3.1.1 Offered Load/Network Load . . . . . . . . . . . . . . . . . . . . . . . 73

B.3.1.2 Application(s) in Use . . . . . . . . . . . . . . . . . . . . . 73

B.3.1.3 UE Antenna Pattern . . . . . . . . . . . . . . . . . . . 73

B.3.1.4 UE-to-UE variation . . . . . . . . . . . . . . . . . . . 73

B.3.1.5 Adherence to Power Control Equation . . . . . . . . . . . . . . . 75

B.3.1.6 Internal Algorithms and Measurements . . . . . . . . . . . . . . . . . . 75

B.3.1.7 Indoor vs. Outdoor $\ldots \ldots \ldots \ldots \ldots \ldots \ldots \ldots$

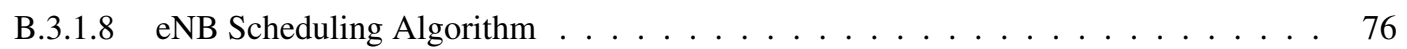

B.3.1.9 Distribution of UEs in a Cell $\ldots \ldots \ldots \ldots \ldots \ldots$

B.3.1.10 Power Control Type . . . . . . . . . . . . . . . . . . . . 76

B.3.1.11 Adjacent Cell Interference ("crosstalk") . . . . . . . . . . . . . . . . . 78

B.3.1.12 Misc. eNB Settings . . . . . . . . . . . . . . . . . . . . 78

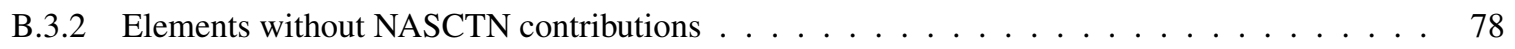

B.3.2.1 How Often the UE is Used . . . . . . . . . . . . . . . . . . . . . . . 78

B.3.2.2 UE Orientation . . . . . . . . . . . . . . . . . . . . . 79 
B.3.2.3 Performance Variation with Temperature and Age . . . . . . . . . . . . . . . 79

B.3.2.4 Propagation Model . . . . . . . . . . . . . . . . . . . 80

B.3.2.5 Dynamic Mobile Environments and Multi-path . . . . . . . . . . . . . . . . . . 80

B.3.2.6 Clutter Type . . . . . . . . . . . . . . . . . . . . . . . . . 80

B.3.2.7 Cell Antenna Pattern and MIMO/Beamforming . . . . . . . . . . . . . . . . . . 80

B.3.3 Factors Not Shown to Contribute to UE Emissions . . . . . . . . . . . . . . . . . . . . . . 80

B.4 Other Considerations . . . . . . . . . . . . . . . . . . . . . . 81

B.4.1 Scheduler Dynamics in Negative Power Headroom . . . . . . . . . . . . . . . . . . 81

B.4.2 Measured vs. Reported Transmit Power . . . . . . . . . . . . . . . . . . . . . . 81

Bibliography 


\section{Acronyms}

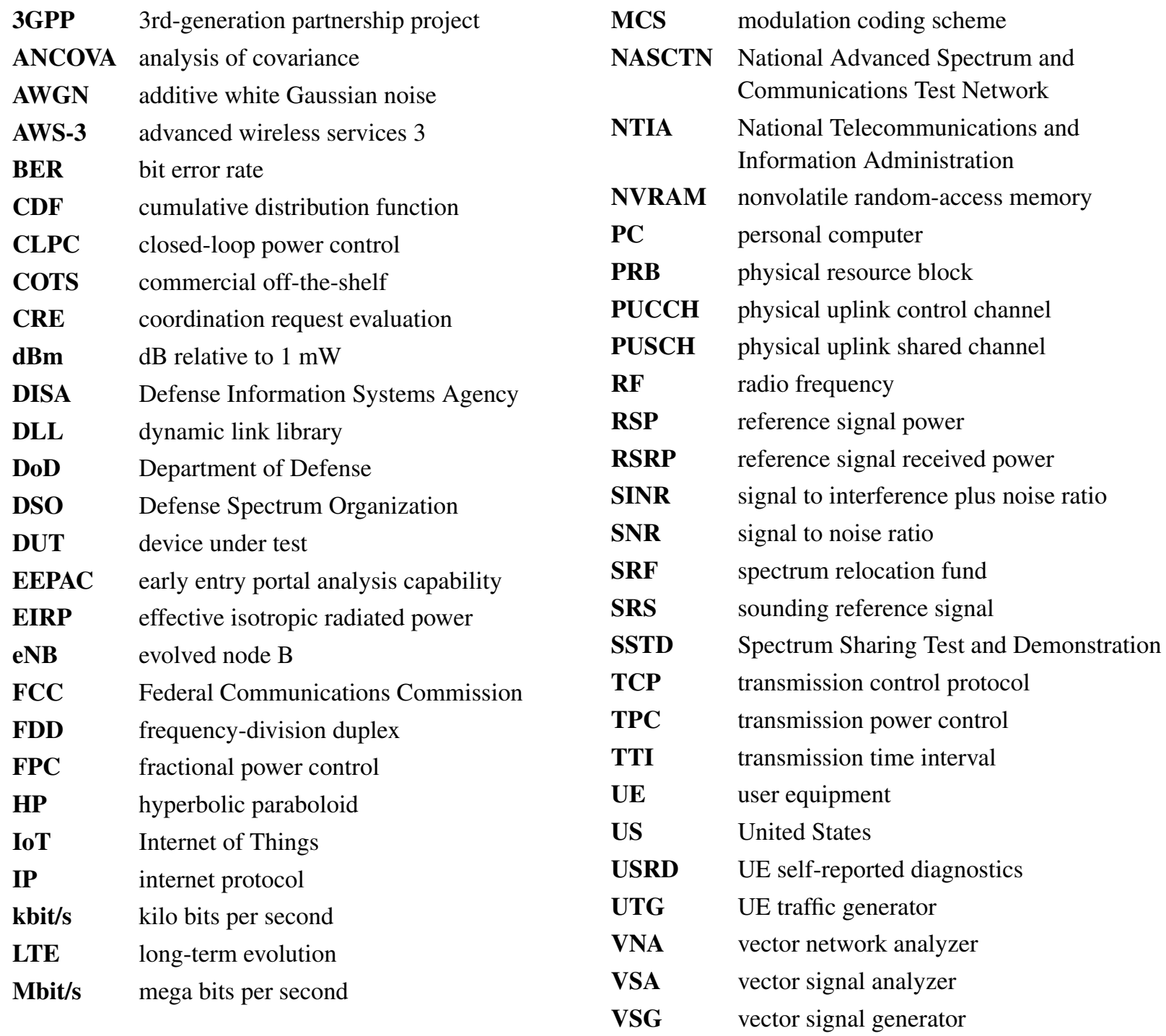




\section{List of Figures}

2.1 eNB Scheduling Areas . . . . . . . . . . . . . . . . . . . . . . . . . . . . . . . . . . . . . . . . 9

3.1 Experimental Setup .......................................... 11

3.2 The spectogram output of the UE traffic generator (UTG) for 4 UEs . . . . . . . . . . . . . . . . . . . . 12

3.3 The spectogram output of the UTG for 8 UEs . . . . . . . . . . . . . . . . . . . . . . . . . 13

3.4 The spectogram output of the UTG for 16 UEs . . . . . . . . . . . . . . . . . . . . . . . . . . 13

5.1 Median and 90th centile of power (measured PUSCH EIRP per PRB) versus applied attenuation for the full modeling experiment data set (top row) and for the modeling experiment data set where the number of active PRBs is greater than 1,000 (bottom row). . . . . . . . . . . . . . . . . . . . . . . . . . . .

5.2 Power (measured PUSCH EIRP per PRB) at $10 \mathrm{~dB}$ applied attenuation at fourteen time points over the two months of the monitoring experiment. The six timeplots correspond to the six combinations of factors $B$ and $C$, crosstalk and offered load. The dark blue points in each plot are medians of the measured PUSCH EIRP per PRB distribution; the light blue points are the 5th and 95th centiles. The plotted points are connected by lines to aid visual assessment of changes in power. . . . . . . . . . . . . . . . . . . . . . . . . . . . . 24

5.3 Power (measured PUSCH EIRP per PRB) at $20 \mathrm{~dB}$ applied attenuation at fourteen time points over the two months of the monitoring experiment. The six timeplots correspond to the six combinations of factors $B$ and $C$, crosstalk and offered load. The dark blue points in each plot are medians of the measured PUSCH EIRP per PRB distribution; the light blue points are the 5th and 95th centiles. The plotted points are connected by lines to aid visual assessment of changes in power. . . . . . . . . . . . . . . . . . . . . . . . . . . . .

5.4 Power (measured PUSCH EIRP per PRB) at $30 \mathrm{~dB}$ applied attenuation at fourteen time points over the two months of the monitoring experiment. The six timeplots correspond to the six combinations of factors $B$ and $C$, crosstalk and offered load. The dark blue points in each plot are medians of the measured PUSCH EIRP per PRB distribution; the light blue points are the 5 th and 95 th centiles. The plotted points are connected by lines to aid visual assessment of changes in power. . . . . . . . . . . . . . . . . . . . . . . . . . . .

5.5 Power (Measured PUSCH EIRP per PRB) at $10 \mathrm{~dB}$ applied attenuation in the monitoring experiment grouped by UE device under test (DUT). The six time plots correspond to the six combinations of factors $B$ and $C$, crosstalk and offered load. The dark blue points in each plot are medians of the measured PUSCH EIRP per PRB distribution; the light blue points are the 5 th and 95 th centiles. $\ldots \ldots \ldots \ldots \ldots \ldots$

5.6 Power (measured PUSCH EIRP per PRB) at $20 \mathrm{~dB}$ applied attenuation in the monitoring experiment grouped by UE DUT. The six time plots correspond to the six combinations of factors $B$ and $C$, crosstalk and offered load. The dark blue points in each plot are medians of the measured PUSCH EIRP per PRB distribution; the light blue points are the 5th and 95 th centiles.

5.7 Power (measured PUSCH EIRP per PRB) at $30 \mathrm{~dB}$ applied attenuation in the monitoring experiment grouped by UE DUT. The six time plots correspond to the six combinations of factors $B$ and $C$, crosstalk and offered load. The dark blue points in each plot are medians of the measured PUSCH EIRP per PRB distribution; the light blue points are the 5 th and 95 th centiles.

5.8 Fitted models of 50th and 90th centiles of measured PUSCH EIRP per PRB are shown (top left, top right) with the monitoring data on which these models are based. Fitted models of the 5th, 50th, 90th, and 95th centiles of measured PUSCH EIRP per PRB based on the data collected in the monitoring experiment are compared (bottom).

5.9 Changepoints in the continuous, piecewise linear relationship between measured PUSCH EIRP per PRB centile response and applied attenuation. The changepoints are roughly the same (around $33.5 \mathrm{~dB}$ ) for the bottom $70 \%$ of the measured PUSCH EIRP per PRB distribution, but is greater by as much as $6 \mathrm{~dB}$ for the top $30 \%$ of the

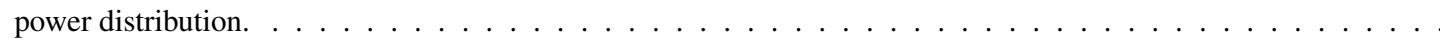

5.10 Fitted HP models of 50th and 90th centiles (top and bottom, resp.) of measured PUSCH EIRP per PRB, shown with the Low crosstalk modeling data on which these models are based. . . . . . . . . . . . . 36 
5.11 Fitted two-piece HP models of 50th and 90th centiles of measured PUSCH EIRP per PRB with the Low crosstalk modeling data on which these models are based. . . . . . . . . . . . . . . . . 38

5.12 Residual plots, RMS errors, and coefficients of determination for the two-piece HP models in Fig. 5.11 and Table 5.9 for the 50th and 90th centile levels (top and bottom, resp.) of measured PUSCH EIRP per PRB. . . . . . 39

5.13 Changelines for the fitted two-piece HP model (5.6) at five centile levels, the 50th and 90th centile levels of the models in Fig. 5.11 and, also, the 5th, 75th, and 95th centile levels. The changelines are overlaid on the combinations of $(A, E)$ design points in the closed-loop power control (CLPC) modeling experiment. . . . . . . . 40

5.14 Elevated variability in the CLPC modeling experiment in 50th and 90th centiles of measured PUSCH EIRP per PRB at High crosstalk (right) relative to Low crosstalk (left) . . . . . . . . . . . . . . . . . . . .

5.15 50th and 90th centiles of measured PUSCH EIRP per PRB at High crosstalk in the CLPC modeling experiment. The elevated variability at High crosstalk remains when the modeling data are grouped by fixed levels of network loading factor $C$, indicating that factor $C$ is not the variability's source. . . . . . . . . . .

5.16 50th and 90th centiles of measured PUSCH EIRP per PRB at High crosstalk in the CLPC modeling experiment. The elevated variability at High crosstalk remains when data are grouped by fixed levels of scheduler type factor $D$, indicating that factor $D$ is not the variability's source. . . . . . . . . . . . . .

5.17 50th and 90th centiles of measured PUSCH EIRP per PRB at High crosstalk in the CLPC modeling experiment. The elevated variability at High crosstalk remains when data are grouped by fixed levels of fractional power

5.18 50th and 90th centiles of measured PUSCH EIRP per PRB at High crosstalk in the CLPC modeling experiment. The elevated variability at High crosstalk remains when data are grouped by fixed levels of FPC power fraction factor $F \ldots \ldots \ldots \ldots \ldots \ldots \ldots \ldots \ldots \ldots \ldots \ldots \ldots$

6.1 Relative Path Loss Calculations versus Factor A: Attenuator Setting . . . . . . . . . . . . . . . . . . . 47

6.2 Plot of Open Loop Expectation. . . . . . . . . . . . . . . . . . . . . . . . . . . . 48

6.3 Comparison of Open Loop Expectation and Measured Power . . . . . . . . . . . . . . . . . . . . . . . . . 49

6.4 Comparison of Expected Power, Measured Power and Open Loop Exploratory Data. . . . . . . . . . . . . . . . 49

6.5 Diagram of Data Flow Showing Aggregation Level . . . . . . . . . . . . . . . . . . . . . . 52

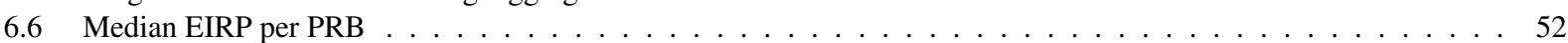

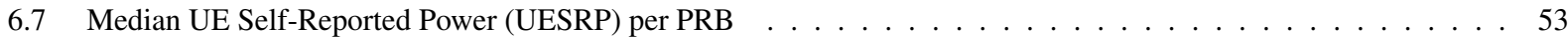

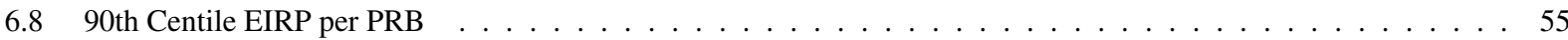

6.990 th Centile UE Self Reported Power (UESRP) per PRB . . . . . . . . . . . . . . . . . . . . . . . . . 55

6.10 Scatter Plot of Measured Versus UE Self-Reported Power (UESRP) per TTI . . . . . . . . . . . . . . . . . 57

6.11 Histogram of Measured - Reported Power per TTI . . . . . . . . . . . . . . . . . . . . . . . 57

6.12 Applied Attenuation Versus RSRP . . . . . . . . . . . . . . . . . . . . . . . . 60

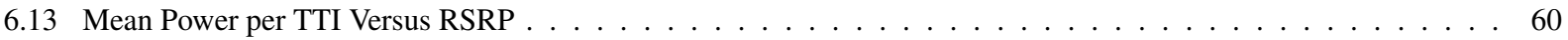

6.14 Mean Power per TTI Versus RSRP by Crosstalk . . . . . . . . . . . . . . . . . . . . . . . . . 61

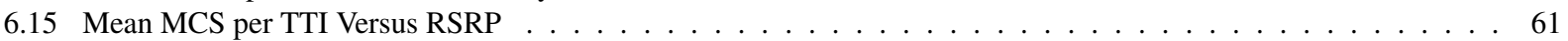

6.16 Mean PRB Grant Size per TTI Versus RSRP . . . . . . . . . . . . . . . . . . . . . . . . . . . 62

6.17 Retransmission Fraction Versus RSRP ～. . . . . . . . . . . . . . . . . . . . . . 62

6.18 Mean MCS index and Mean PRB Size Versus RSRP for Low Crosstalk . . . . . . . . . . . . . . . . . . . 63

B.1 Distribution of EIRP measured from all UE's. . . . . . . . . . . . . . . . . . . . . . . . . . . . . . . . 74

B.2 Summary of empirical CDFs for measured PUSCH power per PRB for all test configurations organized by power control type and testbed attenuation setting. Only data from the factor screening measurements are shown. $\quad$. . . 77 


\section{List of Tables}

2.1 Experimental Settings $\ldots \ldots \ldots \ldots \ldots$

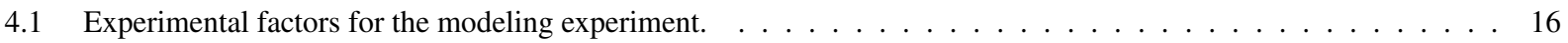

4.2 Split-plot experimental configurations for the modeling experiment. . . . . . . . . . . . . . . . 17

4.3 Whole-plot experimental configurations for the modeling experiment. . . . . . . . . . . . . . . 18

4.4 Split-plot experimental configurations for the monitoring experiment. . . . . . . . . . . . . . . . . . 19

4.5 Test execution matrix for the modeling and monitoring experiments. . . . . . . . . . . . . . . . . . 20

5.1 Monitoring data analysis of covariance (ANCOVA) modeling of the 50th centile of measured PUSCH EIRP per PRB. Table entries in boldface are estimated standard deviations $\hat{\sigma}$ (in $\mathrm{dB}$ ) of the random contribution of handset to the 50th centile of PUSCH EIRP per PRB variability. Corresponding entries in plainface are the associated

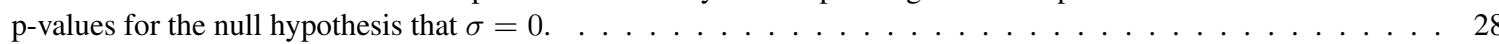

5.2 Monitoring data ANCOVA modeling of the 90th centile of measured PUSCH EIRP per PRB. Table entries in boldface are estimated standard deviations $\hat{\sigma}$ (in $\mathrm{dB}$ ) of the random contribution of handset to the 90th centile of PUSCH EIRP per PRB variability. Corresponding entries in plainface are the associated p-values for the null

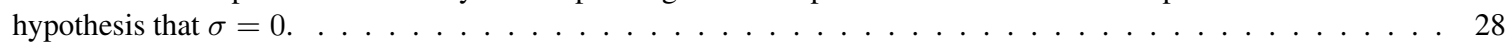

5.3 Monitoring data ANCOVA modeling of the 50th centile of measured PUSCH EIRP per PRB to assess the effect of offered load. . . . . . . . . . . . . . . . . . . . . . . . . . . . . . . . . . 29

5.4 Monitoring data ANCOVA modeling of the 90th centile of measured PUSCH EIRP per PRB to assess the effect of offered load. . . . . . . . . . . . . . . . . . . . . . . . . . . . . . . . . . . . 29

5.5 Continuous, piecewise linear models of select centiles of measured PUSCH EIRP per PRB. Parameter estimates of $\beta_{0}$ and $\beta_{2}$ and their standard errors are in reported in decibels. Parameters $\beta_{1}$ and $\beta_{3}$ are slopes; their estimates and standard errors are dimensionless. . . . . . . . . . . . . . . . . . . . . . . . . . . . 32

5.6 Results for the effects represented in the ANCOVA model (5.4). Effects with p-values below the 0.05 significance level are considered to be discernibly different than zero. . . . . . . . . . . . . . . . . . 34

5.7 Comparison of common and subset descriptive HP models for the 50th and 90th centile measured PUSCH EIRP per PRB responses. . . . . . . . . . . . . . . . . . . . . . . . . . 35

5.8 Fitted HP models for the 50th and 90th centile measured PUSCH EIRP per PRB responses plotted in Fig. 5.10. . . 35

5.9 Fitted two-piece HP models for the 50th and 90th centile measured PUSCH EIRP per PRB responses plotted in

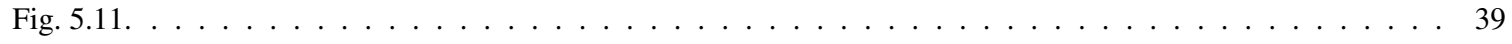

6.1 Two-level factors and settings from the screening experiment results reported in [1] with the largest effect on median EIRP PUSCH per PRB . . . . . . . . . . . . . . . . . . . . . . . . . . . . . . . . . . . . .

6.2 Table of Relative Path Loss Values with 95 Centile Confidence Intervals ～. . . . . . . . . . . . . . . . . . 47

6.3 Median of Median Measured EIRP and USRD per PRB for the Monitoring Experiment . . . . . . . . . . . . . . 54

6.4 Median of Median Measured EIRP and USRD per PRB for the Modeling Experiment . . . . . . . . . . . . . . . 54

6.5 Median of 90th Centile Measured EIRP and USRD per PRB for the Monitoring Experiment . . . . . . . . . . . 56

6.6 Median of 90th Centile Measured EIRP and USRD per PRB for the Modeling Experiment . . . . . . . . . . . . 56

A.1 Sources of uncertainty and uncertainty budget for VSA captures of UE emissions. . . . . . . . . . . . . . . 69

B.1 Characteristics of descriptive, explanatory, and predictive models. . . . . . . . . . . . . . . . . . 70

B.2 Categories of potential model elements (bold) and individual elements, listed below categories. . . . . . . . . . 72 


\section{Chapter 1}

\section{Introduction}

The closed loop power control study presented here was chosen as a characterization test following the initial factor screening experiment executed by National Advanced Spectrum and Communications Test Network (NASCTN) [1]. The goal of this experiment and the previous factor screening campaign was to examine what factors impact the emissions from a commercial off-the-shelf (COTS) user equipment (UE) operating in a long-term evolution (LTE) network. The factor screening experiment identified several factors that strongly affected median uplink power emissions from user equipment. The potential space for variation exceeded the test design of the program. Hence the test campaign chose a subset of conditions that are most likely to yield results that could be used as the basis of a descriptive model of uplink emissions from UEs. As a guide, it was observed that the full variation of behavior for UE uplink power was drastically reduced in closed loop power control. In order to further the community's understanding of the behavior of UE uplink emissions in closed loop power control, two complementary studies were performed. One was a monitoring experiment designed to assess the stability of the test setup and the impact of different UEs of the same model. The second was a modeling experiment designed to yield data as a basis for developing a descriptive model. Although each of these experiments had different aims, much of the uplink emission data yielded similar or complimentary conclusions.

Though the methods and results discussed here specifically relate to a particular frequency band of interest, the methods shown are portable to other frequency bands and for other types of equipment (e.g., narrow-band Internet of Things (IoT) type devices). The results shown here are meant to -in part- inform future analyses of emissions and are not intended to define thresholds for interference or best practices for managing wireless network deployments.

\subsection{Objective and Scope}

The objective of this measurement effort is to characterize the uplink PUSCH power variations of a COTS UE while operating under a closed-loop power control regime, over a range of informed operating conditions. To conduct this investigation, the NASCTN team designed and performed a statistical characterization experiment within the measurement effort. Though not exhaustive, six factors ${ }^{1}$ thought to have the highest likelihood on impacting UE uplink emissions were selected for examination. This study was limited to estimates of the variables, based on laboratory measurements, for analyzing the effects of key variables that can affect the UE behavior under closed loop power control conditions. In-field measurements were not considered as a part of this process. Future studies could extend this plan to include field tests.

This study specifically addresses the characterization of LTE frequency-division duplex (FDD) signals emitted from COTS UEs in the $1755 \mathrm{MHz}$ to $1780 \mathrm{MHz}$ band. The test team expanded on measurement methodology and uncertainty characterization detailed in the prior factor screening report [1].

In addition to characterizing emissions, this measurement effort also further explored the relationship between selfreported transmit power of a UE and actual radiated power. Self-reported power is commonly used during in-field

\footnotetext{
${ }^{1}$ These factors, described in Chapter 2 are additional path loss via applied attenuation, cell-to-cell crosstalk, offered load, uplink scheduling algorithm, nominal PUSCH power, and the path loss compensation factor.
} 
measurements, but its accuracy has not well understood. As a result of the NASCTN test circuit's design, the acquisition of self-reported power was trivial. The comparison between self-reported power and the actual power radiated by the UE was performed as a separate step in the analysis of the data. These collected data sets also proved useful for providing insight into other engineering questions.

\subsection{Background}

In the 2010 Presidential Memorandum on Unleashing the Wireless Broadband Revolution [2], the National Telecommunications and Information Administration (NTIA) was tasked to identify underutilized spectrum suitable for wireless broadband use. In the subsequent NTIA Fast Track Report [3], many federal bands were identified as commercially viable. From this report, the Federal Communications Commission (FCC) identified $1695 \mathrm{MHz}$ to $1710 \mathrm{MHz}$, $1755 \mathrm{MHz}$ to $1780 \mathrm{MHz}$, and $2155 \mathrm{MHz}$ to $2180 \mathrm{MHz}$ together as the advanced wireless services 3 (AWS-3).

Through Auction 97 [4], the FCC auctioned the AWS-3 band for commercial mobile broadband usage in the United States. The auction raised \$41B in revenue for the United States Treasury and required federal agencies in the AWS-3 band to look for other ways to accomplish their missions. In the $1755 \mathrm{MHz}$ to $1780 \mathrm{MHz}$ portion of the AWS-3 band, the Department of Defense (DoD) is using a combination of sharing, compression, and relocation to other bands (including the $2025 \mathrm{MHz}$ to $2110 \mathrm{MHz}$ band).

From the AWS-3 auction proceeds, a spectrum relocation fund (SRF) was created. The DoD is using portions of the SRF to implement its approved transition plans. The SRF is also funding evaluation of early entry coordination requests from the auction winners in the AWS-3 band through the DoD early entry portal analysis capability (EEPAC), which is managed by Defense Information Systems Agency (DISA) Defense Spectrum Organization (DSO). The portal receives requests from auction winners to enter band(s) before the DoD has transitioned out of the bands. These requests must be considered carefully and impartially to deliver a fair answer. If early entry is granted and there is interference to DoD users, it would be very costly to the DoD both financially and in terms of mission execution. If early entry is denied to a commercial carrier for overly conservative reasons, it could be very costly to their business model. To avoid these costs, it is crucial that the findings of the EEPAC are fair and based on a well-understood and openly documented methodology.

The initial test outlined a statistical factor-screening experiment with the primary aim of determining which factors have a significant impact on the distribution of physical uplink shared channel (PUSCH) power per physical resource block (PRB). This experiment, which involved 28 factors, was designed to screen a large number of factors with a practical number of tests, taking into account commercial and laboratory equipment limitations and stability ${ }^{2}$. That study produced the following items:

- Distributions of effective isotropic radiated power (EIRP) from a UE in an active resource block for a variety of test configurations (e.g., applied attenuation/path loss, UE settings, and LTE network settings).

- Includes distributions for PUSCH, physical uplink control channel (PUCCH), and sounding reference signal (SRS).

- A comparison between the UEs self-reported transmit power and measured power distributions.

- 3-D Radiation pattern measurements of several common LTE UEs.

- Uncertainty analysis that includes an uncertainty budget and calculation of overall measurement uncertainty.

At the end of the factor screening work, there were 14 different paths identified by NASCTN for additional exploration. Each of these possibilities is discussed in the factor screening report. After the publication of that report, NASCTN engaged DSO SSTD in a conversation to determine which of those 14 paths would be most valuable to them and the larger stakeholder community. These conversations narrowed the field of 14 down to three different investigations:

\footnotetext{
${ }^{2}$ The summary statistics that encapsulate the measurements performed as part of the factor screening campaign are publicly available at: https://doi.org/10.18434/M32130
} 
detailing UE behavior in a negative power headroom environment, examining the variations in emissions behavior across a sampling of the market, and better understanding the factors at play when closed loop power control is used.

Additional conversations zeroed in on an investigation of UE emissions in closed-loop power control conditions. To not entirely discount the other two final contenders, the closed loop power control characterization experiment was designed to provide a limited amount of insight into UE-to-UE variation (by using four different serial numbers of the same UE model) and to the behavior of a UE in negative power headroom conditions. These additional insights though limited - are still valuable to the community as they provide empirical data and insights not previously available. They can also serve as guidance for others looking to investigate the remaining paths NASCTN identified in the factor screening report.

\subsection{Report Overview}

The remainder of this report is laid out as follows. Chapter 2 provides an in-depth discussion of each of the six experimental factors identified and considered in these measurements. The definition of each factor is given along with a hypothesis of how it may impact the uplink emissions of the device under test (DUT) UE under closed-loop power control conditions. Chapter 3 details the physical test circuit and provides details on how the data are acquired and processed ahead of statistical processing. The salient features of the hardware used in the measurement are provided along with basic information on how the data are acquired. Chapter 4 discusses the statistical experiment design. The statistical analysis and its results are detailed in Chapter 5. In Chapter 6 an engineering analysis is provided. In this analysis, a discussion on the "real-world" impacts of these results is shown along with some exploratory data. The exploratory data provides insight into the comparison between UE reported metrics and independently verified values. The report concludes with a summary of the results, and ideas for future work that expand on these measurements. 


\section{Chapter 2}

\section{Experimental Factors}

The development of the original NASCTN test plan [5] contained an initial list of experimental factors influencing the UE's uplink emissions. After inclusion in the initial test plan, feedback obtained in the public comment period refined that list. The final list of factors used in factor screening was then prioritized into a set of 28 factors given the time and execution constraints [1] .

From the factor screening experiment, between 14 and 20 factors were identified as statistically significant to the UE's uplink emissions ${ }^{1}$. To select the sub-set of factors for the closed-loop power control characterization experiment, the list of statistically significant factors were examined to determine which could have an impact on UE uplink emissions when a closed-loop power control scheme is employed by the eNB.

As in the factor screening process, there were time and execution constraints. One could argue that to be thorough, an experiment involving all statistically significant factors should be designed and executed. However, doing so would take an impractical amount of time while likely not yielding significant additional technical value. Instead, an engineering analysis on possible factors was conducted.

Where as the factor screening process was an effort to identify all statistically significant possible factors, and thus occasionally relied on unrealistic settings (e.g., fractional power control set to 0.5 ) to aid in identifying them, realistic factors and values were sought for this characterization experiment. Given the goal of creating a descriptive model between the factors and emissions, a focus on realistic factors and settings is necessary.

Using both statistical significance and engineering analysis, a decision was made to focus on six factors: applied attenuation (i.e., reference signal received power (RSRP)), crosstalk between cells, the cumulative load offered by UEs to the eNB, the eNB's scheduling algorithm, the nominal PUSCH power at the eNB $\left(P_{0}\right)$, and the fractional power control $(\alpha)$. These six factors frequently appear as parameters in emissions models, are the subject of in-field measurements, and from an engineering perspective could conceivably have an impact on uplink emissions when closed loop power control is used.

The subsections below detail the definition of each of the six factors and the values used in these experiments. Table 2.1 shows a consolidated list of all six factors and their values ${ }^{2}$. Some factors have one set of values for the monitoring experiment and one set for the modeling experiment. This is intentional and discussed in detail in Section 4.2.

\subsection{Factor Definitions}

\subsubsection{Factor A - Applied Attenuation/Path Loss}

From a conceptual perspective, it is easy to see how the amount of path loss between the UE and evolved node B (eNB) can impact uplink power. As the path loss increases (e.g., the UE moves from outdoors to indoors), the UE will need

\footnotetext{
${ }^{1}$ The number of significant factors varies based on the statistical metric used - mean, spread, and skew. Refer to Section 7.2.2 of [1] for additional information

${ }^{2}$ Table 2.1 is also shown as Table 4.1 in Section 4.2. It is shown in both locations as a convenience to the reader.
} 
Table 2.1: Experimental Settings

\begin{tabular}{|c|l|c|c|}
\hline Identifier & \multicolumn{1}{|c|}{ Factor } & Modeling Experiment & Monitoring Experiment \\
\hline A & Additional Path Loss $(\mathrm{dB})$ & $5,10,15,20,25,30,35,40$ & $\begin{array}{c}0,5,10,15,20,25,30,35,37.5, \\
40,42.5,45\end{array}$ \\
\hline B & Crosstalk & Low, High & Low, High \\
\hline C & Offered Load & $10 \%, 20 \%, 40 \%$ & $10 \%, 20 \%, 40 \%$ \\
\hline D & UL Scheduling Algorithm Type & Channel Aware, Interference Aware & Channel Unaware \\
\hline E & Nominal PUSCH $P_{0}(\mathrm{dBm})$ & $-80,-85,-90,-95,-100,-105$ & -85 \\
\hline F & Path loss compensation factor, $\alpha$ & $0.8,1.0$ & 0.8 \\
\hline
\end{tabular}

to transmit more power to reach the eNB. Path loss and UE transmission power are generally positively correlated. The reason for this increase is that the UE is required to maintain the same power at the plane of the eNB receiver regardless of its location. The amount of power desired at the plane of the eNB is known as $P_{0}$, represented by factor E. The eNB - UE radio frequency (RF) link is designed to adapt to changes in the RF conditions while maximizing the UE's battery life and minimizing the inter/intra-cell interference for the data channel and control channel, respectively. This is achieved by not transmitting more power than is necessary to close the link. As part of this process, the eNB and UE frequently exchange information and attempt to estimate the propagation channel conditions. This behavior occurs in real-world deployments as well as in the measurements described here.

In these measurements, the path loss was controlled by use of a variable attenuator inserted between the DUT UE and the eNB. It is recognized that real RF propagation channels between a UE and eNB are almost always dynamically changing, involving some degree of RF fading. Fading can be described as a collection of path losses in various time, location, and radio frequency dimensions. During fading, the propagation path can change rapidly, leading to rapid changes in the power emitted by the UE. Accurately capturing and statistically analyzing a rapidly changing UE power would have made these measurements impractical by significantly increasing the number of factors. Path loss would no longer be a single variable, rather it would be several variables: free-space path loss, rate of change in dynamic fading, depth of RF fades, etc.

The applied attenuation factor used in this characterization experiment can also account for the UE and eNBs antenna pattern. Given the hybrid radiated/conducted measurement circuit, there is no way to explicitly account for any antenna pattern of the $\mathrm{eNB}^{3}$. In cases where a UE was in a null or side-lobe of an eNB antenna, it would artificially increase the path loss between the UE and eNB. With indoor conditions excluded, the applied attenuation variable represents the geographic distance between the UE and eNB. Here, the applied attenuation is set between 8 factor levels ranging between 5 and $40 \mathrm{~dB}$ for the modeling experiment or 12 levels ranging from 0 and $45 \mathrm{~dB}$ for the monitoring study. These values correspond to the effective proximity of the UE to the eNB, where small values of the attenuator $(0,5$, $10 \mathrm{~dB}$ ) represent a small change in distance and large values (30 dB or greater) represent a relatively far increase in distance.

This effective distance also manifests itself in several different engineering parameters of the UE - eNB system. First, it changes the total path loss of the uplink and downlink communication channels simultaneously. This change is reflected in a decrease of the RSRP for an increase in path loss. In addition, it changes the downlink path loss estimate the UE uses to calculate its power output according to the power control equation. Although each of the relative changes of the applied attenuation are known in each whole plot configuration to a great degree of precision (typical

\footnotetext{
${ }^{3}$ The DUT UE was intentionally oriented to maximize coupling to the receive antenna placed in the anechoic chamber
} 
less than $0.17 \mathrm{~dB}$ difference) the absolute difference between any two whole plot configurations (or changes in UE or eNB configuration) can be 2-3 dB in RSRP or $>7 \mathrm{~dB}$ in estimated UE downlink path loss. Details on this phenomenon can be found in Section 6 .

\subsubsection{Factor B - Crosstalk}

The crosstalk factor is defined as the amount of signal from an adjacent cell (referred to here as "Cell B") that is impinging on our cell of interest (referred to as "Cell A"). The hypothesis is that even under closed loop power control conditions, interference or signal from another cell could cause the UEs to transmit more power. In essence, the interfering signal could be raising the noise floor of the serving cell, which would cause the closed loop power control algorithm to increase the power from the UE in order to maintain a constant signal to noise ratio (SNR) or bit error rate (BER).

It's important to note that the interference being presented to Cell A from Cell B is "structured" interference; it is the live LTE signal. This may or may not accurately represent real-world adjacent cell or interfering signals. Interfering or adjacent cell signals have also been represented as noise-like signals in other studies

In this study, cross talk was implemented in a two-step process. First, the genreation of the LTE signal, and second an attenuator controlling the amplitude of the generated LTE signal impinging on Cell A. The crosstalk LTE signal is generated by changing the number of loading UEs in cell B. The number of loading UEs is changed from 4 UEs in the crosstalk low condition to 16 UEs in the crosstalk high condition. Each UE is a simulated UE from the UTG, and has a data rate of $500 \mathrm{kbps}$ and a RSRP of $-95 \mathrm{dBm}$.

To control the amplitude of the signal impinging on Cell A, the Cell B LTE signal discussed above is passed through a variable attenuator on its way to Cell $\mathrm{A}$. This inter-cell leakage path isolation is controlled by a variable attenuator that is set to $110 \mathrm{~dB}$ in the crosstalk low case and $35 \mathrm{~dB}$ in the crosstalk high case. The leakage path isolation was chosen such that a SINR change of $10 \mathrm{~dB}$ was measured by the eNB's receiver in the case of high crosstalk. We disabled any advanced LTE intercell interference features to better measure the effect of crosstalk on the DUT.

In this study, the amplitude of the crosstalk was set to a fixed value prior to the measurement campaign. While Cell A was being subjected to the crosstalk signal the SNR as reported by the eNB was monitored. A variable attenuator was adjusted until the eNB reported a drop of $10 \mathrm{~dB}$ in its SNR (as compared to having no interfering signal present). During the measurement campaign, the crosstalk is controlled by a variable attenuator between the cells. Details on this RF path can be found in Section 3.1.

\subsubsection{Factor C - Network Offered Load}

The network offered load can effect the cell scheduler on its resource allocations decision to each UEs in a cell. Therefore, the UE's power transmission may be affected as well. We select the cell loading to be 10\%, $20 \%$ or, $40 \%$ of the theoretical throughput of a $20 \mathrm{MHz}$ LTE channel. The network offered loading in the serving cell (Factor C) was controlled by introducing a variable number of loading UEs (i.e. 4, 8, and 16 UEs) with the same the offered data rate of $500 \mathrm{kbps}$. These loading UEs are in addition to the one DUT UE that is also active in the serving cell.

One possible impact of a high number of loading UEs transmitting simultaneously could be the RF noise in the LTE channel is increased. To compensate for this, the DUT UE may increase its power to maintain a given SNR. A potential secondary impact is that the loading UEs create a significantly different network loading condition that causes the eNB to change the way it allocates resources to the DUT UE. This change may affect the UE's uplink power. 
These measurements only test the latter of these two cases because the UE traffic generator (UTG) is simultaneously emulating the traffic associated with all of the loading UEs. Though the UTG is emulating the physical layer traffic of the loading UEs, it may not accurately reflect its combined spectrum. This effect is discussed in more detail in Section 3.1.1. Since this spectral inaccuracy could potentially bias the results, the test circuit was designed to isolate the spectrum of the loading UEs from the DUT UE's spectrum.

In the "high" value condition, the number of loading UEs was selected to load the network to full capacity without pushing it into extreme/unusual behavior. During pre-test activities, it was noted that severely overloading the eNB's scheduler ${ }^{4}$ resulted in behavior that was not consistent with its normal operation.

\subsubsection{Factor D - Uplink Scheduling Algorithm}

For factor D, there are three different uplink scheduling algorithms: channel aware, interference aware, and channel unaware. The objective of channel aware scheduling is to allocate more resources to UEs in favorable RF conditions while not starving the UEs that happen to be near the cell edge. Interference aware scheduling aims to reduce the effect of interference (from any source) by allocating resources in parts of the channel less impacted by interference. These allocations can be made based on a varitey of metrics. For example, the eNB may sample the channel and attempt to detect interference itself. Both channel aware and interference aware scheduling methods rely on some information on the RF channel; be it from RF sampling, reports from the UE, or other metrics as measured by the eNB (e.g., PER). The channel unaware scheduling method schedules resources evenly among UEs in the cell in frequency or time domain. The channel unaware scheduling method is also known as the "Round Robin" method [6].

In the monitoring experiment, the uplink scheduling algorithm was set to channel unaware, since that is the default setting on the eNB used for the experiments. For the modeling experiment, the scheduling algorithm was set to either channel aware or interference aware. This choice was made for two reasons. First, since those algorithms are designed to be sensitive to conditions in the RF channel, which is influenced by both the applied attenuation and crosstalk factors, it was desired to assess the impact of those schedulers. Second, the channel-sensitive schedulers were used in the modeling experiment since the channel unaware scheduling algorithm was already evaluated in the monitoring experiment.

\subsubsection{Factor E - Cell Specific Nominal Power Factor}

This factor represents the cell specific nominal power (also known as $P_{0}$ ) to be used in the power control equation for UE uplink transmission power calculations. This can also be described as the desired power level of uplink signals at the plane of the eNB's receiver. In the absence of inter-cell interference, the higher the $P_{0}$ value, the better the throughput and reception at eNB with tradeoffs, e.g. increased inter-cell interference. This value of this factor is typically configured in the field by cellular operators and is informed by the local conditions of RF and cell coverage.

\subsubsection{Factor F - Fractional Power Control}

The fractional power control factor, $\alpha$, is used to adjust the path loss value in the calculation of uplink power (in either closed- or open-loop power control schemes). It is applied to the path loss value measured by the UE from the downlink reference signal. In real-world deployment scenarios, the value of $\alpha$ can be adjusted to overcome variations found in that particular propagation environment. This compensation factor can mathematically have a range from 0.0 to 1.0 , however in practice, the value is typically between 0.8 and 1.0. When $\alpha$ is set to 0.0 , the path loss is ignored

\footnotetext{
${ }^{4}$ Unusual behavior was observed with greater than 100 simultaneous active UEs in the cell. This behavior may also be present at lower values; extensive investigation into the anomalous behavior was not conducted.
} 
in the UE's power control calculation and when $\alpha$ is set to 1.0, fractional power control is said to be disabled. When fractional power control is disabled, the UE's power will attempt to maintain a constant signal to interference plus noise ratio (SINR) at the eNB. Under this scenario, if the path loss increases by $10 \mathrm{~dB}$, so will the UE's transmit power to keep a constant SINR value at the eNB's receiver.

When $\alpha<1.0$, fractional power control is enabled. When fractional power control is enabled, the SINR at the eNB is allowed to decrease as path loss increases. In this scenario, as path loss between the UE and eNB increases, it is only compensated for by a fractional amount (mathematically between 0.0 and 0.99 ). The specification for fractional power control can be found in 3rd-generation partnership project (3GPP) Technical Specification 23.213 [7]. In this investigation we set $\alpha$ to 0.8 or 1.0. These values were selected based on experience discussing deployment scenarios with operators.

\subsection{Collateral eNB Factors}

In the process of configuring the eNB with the above eNB parameters, additional eNB parameters needed to be modified from their default values for the eNB to have a valid configuration. These collateral factors were not analyzed as part of the experiment. In this context we treat their contribution to UE uplink emissions under closed loop power control conditions as an unknown.

Sounding Reference Signal The sounding reference signal (SRS) is an LTE feature that is used to give an eNB a more accurate picture of the propagation channel between each UE in the cell and the eNB. This feature is a configurable parameter and it is closely related to other eNB parameters. In particular, the activation of SRS is required when the scheduling methods "channel aware" or "interference aware" are used. For "channel unaware" scheduling this parameter must to be turned off.

Because the SRS feature includes the physical transmission of energy in the uplink, it does increase the emissions from the UE. However, in terms of the number of overall resource blocks used, SRS represents a very small fraction of the total number of PRBs used in an LTE cell; occupying up to four RBs per SRS transmission. The location and hopping sequence of the SRS are broadcasted by the eNB. To avoid collision and improve detection, the SRS power offset is broadcast by the eNB to all UEs and each UE given a specific subframe to transmit its SRS.

eNB Uplink Scheduling Area The uplink shared channel bandwidth can be divided into as few as two or as many as six areas by the eNB when it's configured for the "channel aware" or "interference aware" scheduling algorithms. When enabled, the eNB will avoid scheduling in specified areas in an attempt to mitigate the effects of interference. In channel unaware scheduling, the eNB scheduler considers the PUSCH area to be one continuous area.

In these experiments, for the number of PUCCH areas for "channel aware" and "interference aware" we used the default value of three (see Figure 2.1). This should not have any impact on the scheduling behavior because, even when configured to three areas, the eNB was still allowed to use all areas. At no point in the measurement campaign did we configure the eNB to avoid a scheduling area through the use of this parameter. In cases of high crosstalk, the eNB may have decided to avoid an area of the channel based on measured noise/interference present in the channel. 


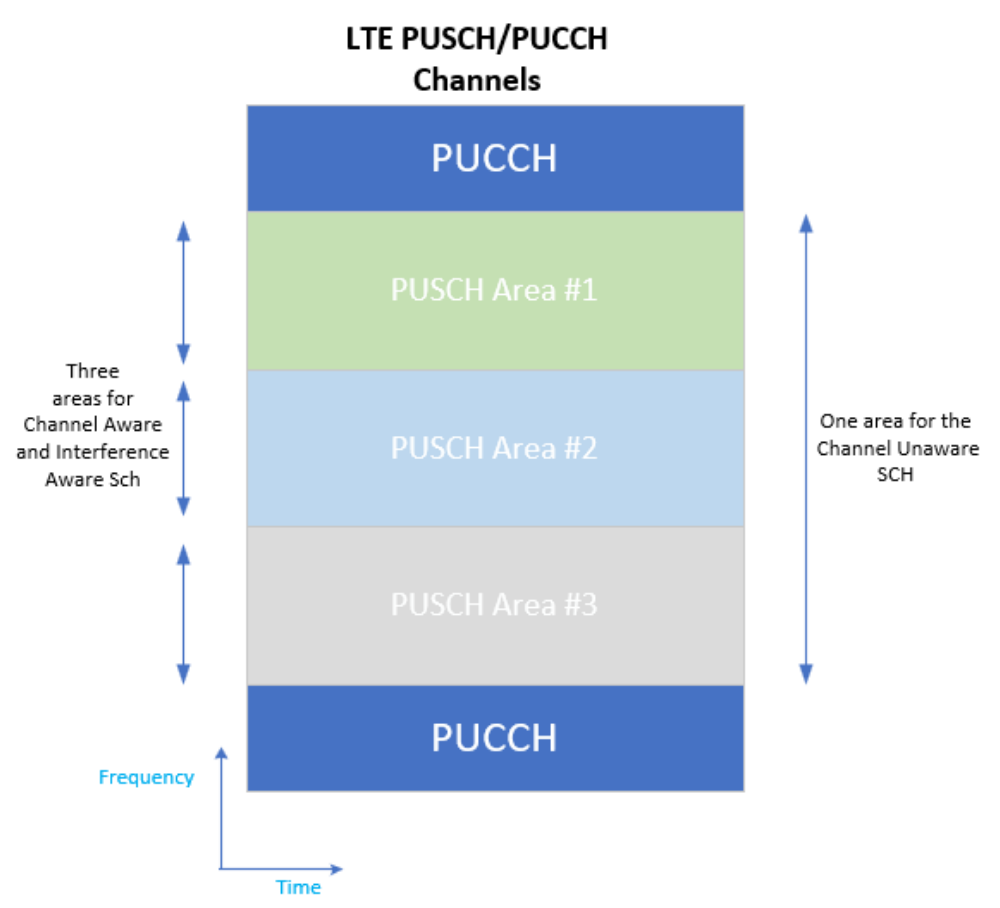

Figure 2.1: eNB Scheduling Areas 


\section{Chapter 3}

\section{Testbed Design}

This chapter outlines the design and implementation of the test circuit used in the closed-loop power control experiments ${ }^{1}$. The test setup contains a set of RF circuitry and hardware, with main elements allowing for signal leveling, an UTG generating loading UE traffic, COTS UE as the DUT, and the eNB. Note that the LTE infrastructure that the eNB is connected to is, for simplicity, omitted here as it is not the subject of this testing. This infrastructure is described in section 3.2.1 of [1]. The method, design, and equipment are discussed in detail both to enhance understanding of the measurements that NASCTN performed and to enable others to recreate the same or a similar test circuit for their measurements.

From a conceptual perspective, this circuit replicates two adjacent LTE cells, "Cell A" and "Cell B". Both cells are populated with a set number of loading UEs (see Section 2.1.3). These loading UEs are generated by the UTG, and behave as though they are real UEs transacting data across all layers of the network. Cells A and B are independent of each other; their X2 interface has been disabled, and UEs in each cell are not permitted to handover from one cell to the other. Though the cells are independent of each other, a controlled amount of energy is permitted to cross from one cell to another under specific test cases (see Section 2.1.2).

The COTS DUT UE is virtually inserted into Cell A. This DUT UE radiates inside a small anechoic chamber. An antenna placed at the top of the chamber receives (or transmits) signals into Cell A. The DUT UE - eNB path loss is controlled by a programmable attenuator. The uplink emissions from the DUT UE are measured using a vector signal analyzer (VSA).

\subsection{Test Circuit}

To characterize the relationship between the six factors outlined in chapter 2, NASCTN developed the test circuit as part of the initial campaign [1]. In general, the two eNB cells can be seen on the right side of the diagram and the UTG generating the loading UEs on the left. In the lower left, a small shielded chamber is shown housing the DUT UE. The circuitry immediately to the right is responsible for inserting the DUT UE into Cell $A$. The circuitry in the middle of the diagram is used to control the crosstalk between cells and shift the level of the downlink signal to a level appropriate for the UTG.

Cell A and Cell B were both implemented with a commercial macro-cell operating in Band $66^{2}$. For these measurements, Cells A and B were locked to an uplink channel that spanned $1770 \mathrm{MHz}$ to $1780 \mathrm{MHz}$ and a downlink channel that spanned 2170 to $2180 \mathrm{MHz}$. All experiments were performed at these frequencies.

The hardware and software design of this experiment follows a previous setup outlined in [1], with the exception of an augmented path to introduce cross talk conditions as highlighted in figure 3.1. The cell loading factor was realized by creating either 4,8 , or 16 loading UEs in cell A. Each loading UE transmitted at a data rate of 500 kilo bits per

\footnotetext{
${ }^{1}$ Specifications not enumerated here or in [1] are not thought to have a meaningful impact on the test results.

${ }^{2}$ By definition, LTE Band 66 is an FDD band with an uplink frequency between $1710 \mathrm{MHz}-1780 \mathrm{MHz}$ and a downlink frequency between $2110 \mathrm{MHz}-2200 \mathrm{MHz}$ [Table 5.5-1 of [8]]
} 
second (kbit/s) with an RSRP of $-95 \mathrm{~dB}$ relative to $1 \mathrm{~mW}(\mathrm{dBm})$. The crosstalk low condition was realized with four emulated UEs in Cell B and the cross talk attenuator (shown in the dashed box in Figure 3.1) set to its maximum setting of $110 \mathrm{~dB}^{3}$. In contrast, the crosstalk high condition consists of 16 emulated UEs in cell B and a path isolation chosen to give a decrease in SINR of $10 \mathrm{~dB}$ as measured by the eNB receiver ${ }^{4}$ Additionally, the effective path loss between the DUT UE and eNB was controlled by a programmable attenuator in the path. This attenuator took on values from $0 \mathrm{~dB}$ to $45 \mathrm{~dB}$, resulting in an effective RSRP of -72 to $-112 \mathrm{dBm}$.

The green element shown to the right of the small shielded chamber and to the left of the VSA is an RF switch. At the beginning of measurement sequences (e.g., after a new eNB configuration was loaded), the system was designed to switch the VSA to a matched load. When switched to the matched load, the only signal present on the VSA is noise or leakage, and thus represents the floor of its measurement capability.

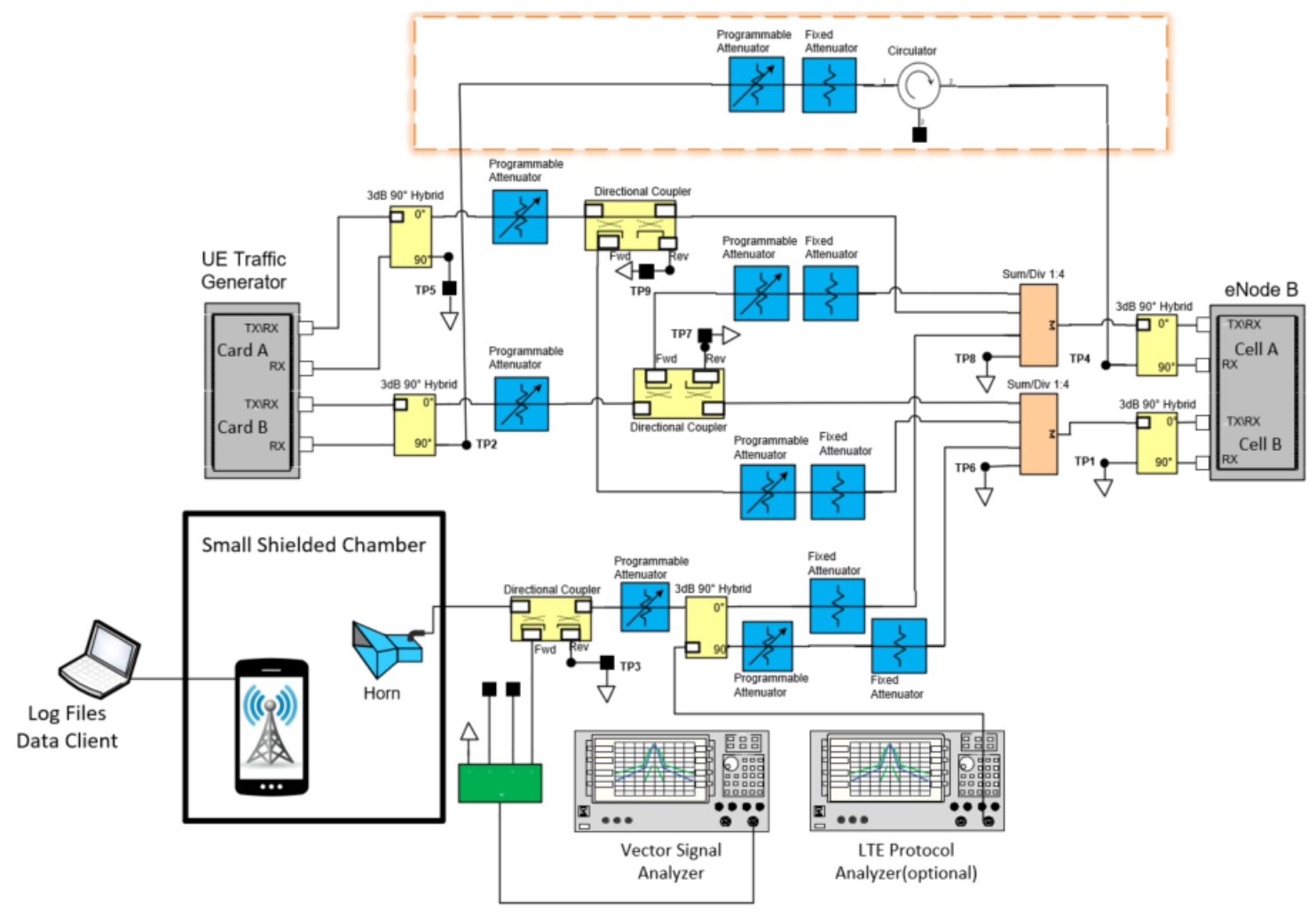

Figure 3.1: Experimental Setup

\subsubsection{UE Traffic Generator}

The UTG device is a laboratory test equipment for loading an LTE system with different types of traffic. This equipment can emulate hundreds of UE within a single chassis. The particular chassis used for these measurements contained two separate radio cards, one for each cell. The independent cards enabled each cell to operate independently of the other. The UTG virtually places each loading UE within the cell at a user defined RSRP (-95 dBm in this case). Once the experiment is underway, the UTG would generate a full stack implementation for each UE - from the physical layer to the application layer. In this case, the application layer consisted of sending randomly generated UDP traffic to an application server on the IP side of the network.

\footnotetext{
${ }^{3}$ Note that due to parasitic leakage through other RF components in the circuit, the actual isolation between the cells in the crosstalk low setting was in excess of $80 \mathrm{~dB}$

${ }^{4}$ This SINR value was reported by the eNB itself. This reported value is averaged over a 15 minute time period.
} 
The advantage of using a UTG is to work and modify the environment of many UEs while monitoring their LTE protocol layers logs for later analysis. The main limit of an UTG is its maximum power transmission of each card. Each card can only transmit $+20 \mathrm{dBm}$. Therefore, when many UEs are emulated, the UTG needs to alter the data content of LTE layers to keep the UEs in the desired configured state. This limitation means that it is crucial that the UTG uplink spectrum is not included in our analysis. The circuitry shown in Figure 3.1 accounts for this by placing the VSA as close to the DUT UE as possible. The isolation between the DUT UE spectrum at the VSA and the loading UEs was found to be within the thermal noise floor of the measurement vector network analyzer (VNA), that is isolation was in excess of $105 \mathrm{~dB}$.

To provide additional insight into how the UTG functions at the physical layer, the test circuit was reconfigured to capture the physical layer output from the UTG. The VSA captured spectrograms of the UTG for 4 UEs can be seen in figure 3.2, 8 UEs in figure 3.3, and 16 UEs in figure 3.4. The light blue color indicates the resources used for the UEs in a transmission time interval (TTI). From the spectrograms, we can see the UTG follows the LTE resource scheduling and settings given the eNB configuration. The power of each resource on the VSA may differ from what the UTG reported to the eNB.

The dynamic range of the RF signals from the UTG are determined when a test configuration is initally set up. The UTG uses this initial value to determine what deviations in power and addition of noise to create the required SNR for the specified path loss based on the additional attenuation applied by the variable attenuator. In some cases, the UTG may modify values reported to the eNB via the LTE protocol to achieve the desired effects. Even when modified, the messages still comply with the $3 \mathrm{GPP}$ specifications. This procedure is the same for Open- or Closed-loop power controls.

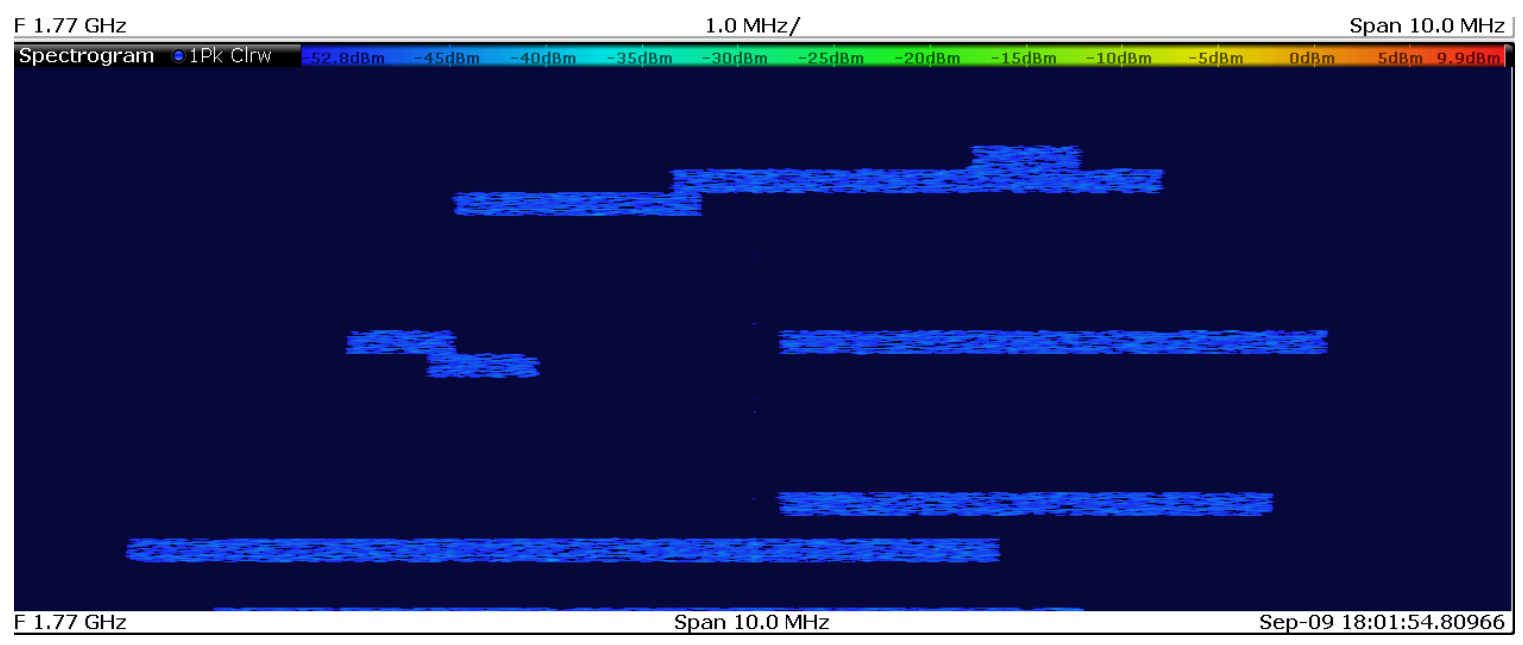

Figure 3.2: The spectogram output of the UTG for 4 UEs

\subsection{Data Sources}

There are three primary sources of data in these experiments: the UE self diagnostics, the spectrogram measured by the VSA and the diagnostic logs produced by the UTG. The UE self diagnostic reports ${ }^{5}$ include a time stamp, system frame number and system subframe number that is analyzed to create an experimental global time basis. Only a portion of all available fields available from the UE self diagnostic report are utilized. The fields required for creating the global time basis, the total transmitted power per TTI, the number of resource blocks allocated, and the start

\footnotetext{
${ }^{5}$ The type of UE self-reported diagnostics (USRD) software used here is different from other applications (or "apps") that can be installed on top of a UE's operating system. Some apps may be able to provide the same or similar information as the USRD software, but it may consume more of the UE's computing resources.
} 


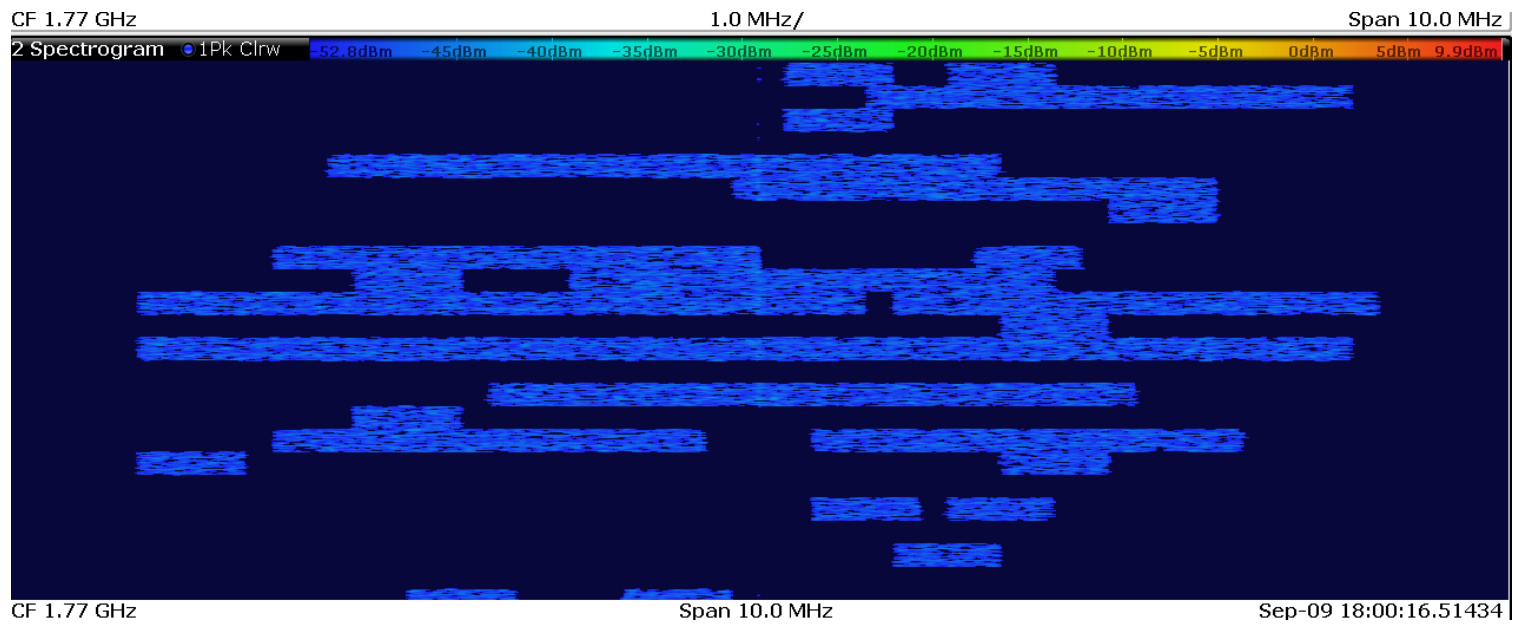

Figure 3.3: The spectogram output of the UTG for 8 UEs

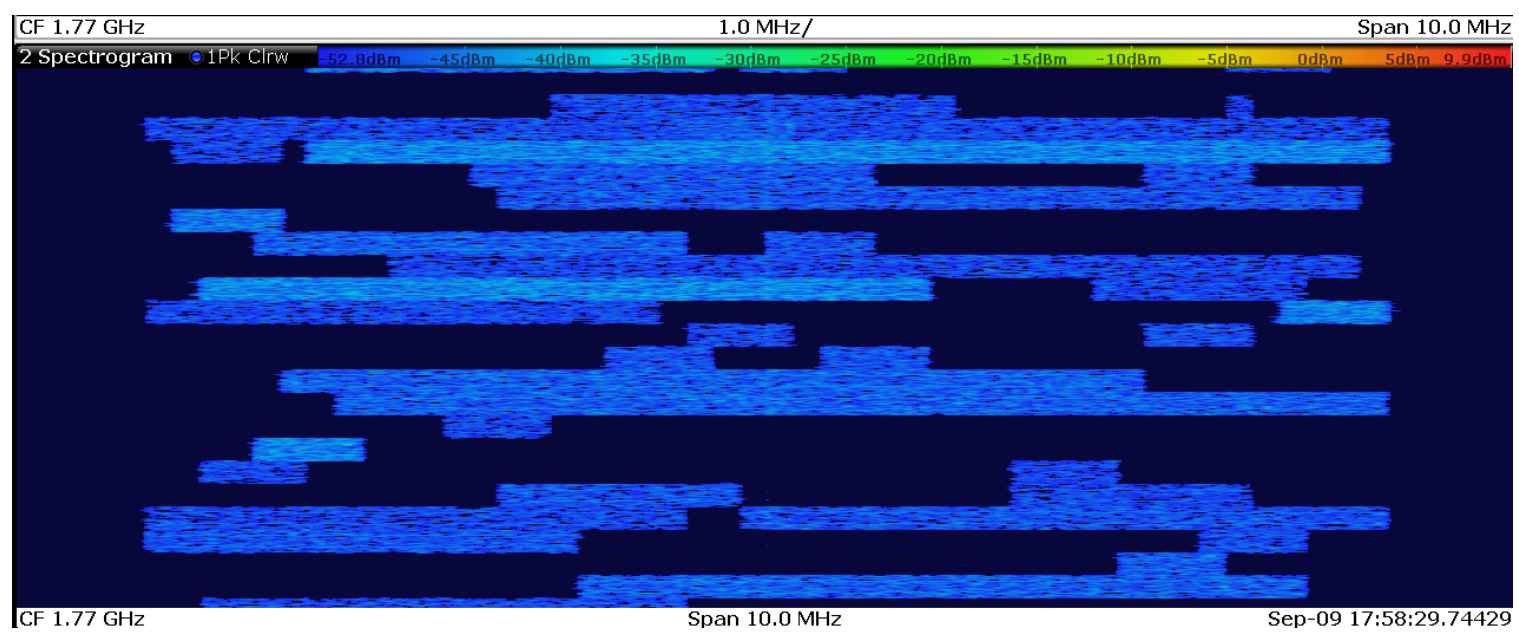

Figure 3.4: The spectogram output of the UTG for 16 UEs

resource block allocated are used to create a synthetic spectrogram. This is used to time align other outputs and mask the data measured by the VSA. This synthetic spectrogram and the acquired VSA spectrogram are aligned using a cross correlation technique. This technique identifies the maximum point of correlation that is then used as a time-shift from the global time basis to the VSA spectrogram time basis that is applied, centering the VSA spectrogram to the global time basis. The in-house produced software that performs this task is covered in detail in [1]. This software was augmented to extract additional UE diagnostic information, in particular the PUSCH power control parameters that include the reported total transmitted power, downlink path loss, transmit power control, and total physical resource blocks.

In addition to the above parameters, the UTG and DUT UE diagnostic reports provided additional parameters. These additional parameters were collected as a means of independently verifying the expected operation of the testbed. The full list of parameters collected in these characterization measurements matches the list from Factor Screening. See Appendix C of [1] for additional information. Values for BSR, MCS, the number of location of PRB grants, and PHR were among those collected for each LTE subframe (1 ms interval). This was particularly useful during the pre-test stages of debugging. Once the measurement campaign commenced, these parameters were checked to ensure each 
configuration was executed as expected.

Similar to the USRD, the UTG also provided additional parameters useful for verification and troubleshooting. Parameters collected from the UTG include the number of currently attached loading UEs, the mean number of UEs signaled per TTI, and the number of RACH attempts ${ }^{6}$. Each of the UTG parameters were collected as an average value over a $500 \mathrm{mS}$ time period.

\subsection{DUT UE Devices}

The closed loop power control characterization experiments were done with four category $18^{7}$ COTS LTE UE devices that were released to the United States (US) market in 2018. All four devices were the same model, but different serial numbers. All four devices were "unlocked", that is, not programmed to be fixed to any single cellular carrier. The software image (i.e., operating system) was updated to the most current version available at the start of the measurement campaign. All four devices had identical versions of software. The devices were not updated during the measurement campaign. Each device was provisioned with a SIM card for the NASCTN laboratory network.

In order to prevent the UEs from attempting to handover or search for other cells, software was used to change a setting within the UE's nonvolatile random-access memory (NVRAM) so that the UE would stay locked on Band 66 and not search for other available frequency bands or cells.

To control the uplink traffic of the DUT UEs, an application designed for generating and sending internet protocol (IP) traffic was installed on each DUT UE. This application was controlled through a developer bridge between the DUT UE and the control personal computer (PC). The connection from the control PC was independent of the wireless LTE connection. As discussed in Section 2.1.3, all of the traffic was of UDP type, and set to continuously send $500 \mathrm{kbps}$ to an application server ${ }^{8}$.

\subsection{Data Acquisition}

To implement the experiments defined in Chapter 4, the measurement setup needed to produce repeatable, meaningful, and applicable data. This meant that the test must proceed through clearly defined states. The full test state for each factor screening test case is described in Section 4.2. Each time an eNB configuration is loaded, the system is initialized to ensure that the system is operating in steady state (though the data may still be dynamic).

The test sequence iterates through all test cases in the defined order. In each test case, a configuration is applied to the hardware in the test setup, and data is acquired and recorded. The iteration is defined as two levels of nested loops:

- an outer loop that sets the eNB test parameters

- an inner loop that sets the other test parameters that are not determined by the eNB

An important practical aspect of this measurement campaign is the overall test execution time. Test execution is most efficient when slow operations are executed in the outer loop. In this implementation, changing the eNB configuration is the slowest single operation, so it is specified in the outer loop. Other test implementations may consider swapping the inner and outer loops if different instruments take longer to configure. The order in which loops are executed should have no impact on the results of the experiment/measurements.

\footnotetext{
${ }^{6}$ As with Factor Screening, a UE RACH attempt during data collection resulted in those data being excluded and the configuration repeated. Due to the configurations used in these characterization, RACH attempts after the first attach were virtually non-existent.

${ }^{7}$ LTE UE category 18 is specified to have a maximum downlink data rate of 1200 mega bits per second (Mbit/s) and a maximum uplink rate of $150 \mathrm{Mbit} / \mathrm{s}$.

${ }^{8}$ This application server is independent from the application server used by the UTG. One application server could be used to serve both the loading UE and DUT UE, but separate servers was much easier to implement.
} 


\section{Chapter 4}

\section{Experimental Design}

\subsection{Overview}

The primary objective of this study was to characterize PUSCH power emissions under closed-loop power control over a range of realistic conditions that could be found in a real-world deployment environment. In particular, the experiment sought to collect data that could be used to develop a descriptive model for PUSCH EIRP per PRB. Secondary goals of this work included assessment of negative power headroom conditions and differences between measured and UE-reported power. Our experimental investigation consisted of two complementary experiments executed in parallel, a modeling experiment and a monitoring experiment.

The modeling experiment was designed to characterize the influence of the six factors listed in Table 4.1. Note that three factors (A, B, C) were non-eNB factors and three factors (D, E, F) were eNB factors. Descriptions and implementation details for each factor are provided in Chapter 2.

Because changing eNB factor settings required more testbed time than changing non-eNB factors, the modeling experiment used an efficient split-plot experimental design $[9,10,11]$ to minimize the number of eNB factor changes. In this design, the eNB factor configurations (whole-plots) and non-eNB configurations (split-plots) were loaded onto the testbed in two nested loops, where the non-eNB configurations were changed in the inner loop and eNB configurations were changed in the outer loop. Following sound experimental practice, the test order of the whole-plots and split-plots was randomized to mitigate effects from uncontrolled factors $[10,11]$. Note that the order of test configurations was not fully randomized, since the structure of a split-plot design requires that the whole-plot and split-plot factors are changed in a nested fashion. To account for this fact, the statistical analysis of the modeling experiment in Section 5.3 includes separate whole-plot and split-plot error terms.

The modeling experiment executed four rounds of testing, where each round consisted of 12 eNB factor configurations (whole-plots) and 48 non-eNB factor configurations (split-plots). Four of same UE model were tested, where a different UE was used for each round. Additional details for the modeling experiment are provided in the next section.

The aims of the monitoring experiment were to assess testbed stability, gauge the impact of different UEs, and explore negative power headroom in fuller detail than allowed by the design of the modeling experiment. To address these goals, the monitoring design, consisting of a single eNB configuration and 72 non-eNB factor configurations, was repeated periodically, at the start of each block of four eNB configurations. The four UEs were sequentially changed between replications in a consistent order; i.e., the handsets were used in the order 1,2,3,4,1,2,3,4,1,2,3,4,2,3. Further experimental details are given in the next section. 
Table 4.1: Experimental factors for the modeling experiment.

\begin{tabular}{|c|c|l|c|}
\hline Identifier & Testbed Component & \multicolumn{1}{|c|}{ Factor } & \# Levels \\
\hline A & Variable Attenuator & Path Loss & 8 \\
\hline B & UTG & Crosstalk (variable attenuator \& adjacent cell UEs) & 2 \\
\hline C & UTG & Offered Load (number of loading UEs) & 3 \\
\hline D & eNB & UL Scheduling Algorithm Type & 2 \\
\hline E & eNB & Power Control: Nominal PUSCH $P_{0}$ & 6 \\
\hline F & eNB & Power Control: Path loss compensation factor, $\alpha$ & 2 \\
\hline
\end{tabular}

\subsection{Design Details}

The settings for the modeling and monitoring experiments are summarized in Table 2.1. Additional details on the selection and implementation of these settings are provided in Chapter 2.

The modeling experiment used full-factorial designs for the eNB and non-eNB factors, respectively, i.e., all combinations of the factor settings were tested. In each round of testing, the design consisted of $12 \mathrm{eNB}$ factor configurations and 48 non-eNB factor configurations. Three levels of factor E, PUSCH $P_{0}$, were tested in each round, with the levels staggered across rounds so that a total of six levels were tested overall.

Split-plot and whole-plot configurations for the modeling experiment are listed in Tables 4.2 and 4.3, respectively. In these tables, the respective settings listed in Table 2.1 are labeled with integers between one and the number of levels. Note that whole-plot configurations 1-12 and 13-24 correspond to staggered levels for factor E, PUSCH $P_{0}$.

The monitoring experiment used a single whole-plot (eNB) configuration and a full-factorial design consisting of 72 split-plot (non-eNB) configurations across the selected settings for factors A, B, and C. As shown in Table 2.1, the monitoring experiment tested twelve levels of factor A, applied attenuation, which was four more than the modeling experiment. The extra levels of factor A were added to the monitoring experiment to gain further insight into $\mathrm{PUSCH}$ emissions at high applied attenuation values, particularly negative power headroom conditions. The 72 split-plot configurations for the monitoring experiment are listed in Table 4.4, where the respective settings are labeled with integers between one and the number of levels. 
Table 4.2: Split-plot experimental configurations for the modeling experiment.

\begin{tabular}{|c|c|c|c|}
\hline Configuration & $\mathbf{A}$ & B & $\mathbf{C}$ \\
\hline 1 & 1 & 1 & 1 \\
\hline 2 & 2 & 1 & 1 \\
\hline 3 & 3 & 1 & 1 \\
\hline 4 & 4 & 1 & 1 \\
\hline 5 & 5 & 1 & 1 \\
\hline 6 & 6 & 1 & 1 \\
\hline 7 & 7 & 1 & 1 \\
\hline 8 & 8 & 1 & 1 \\
\hline 9 & 1 & 2 & 1 \\
\hline 10 & 2 & 2 & 1 \\
\hline 11 & 3 & 2 & 1 \\
\hline 12 & 4 & 2 & 1 \\
\hline 13 & 5 & 2 & 1 \\
\hline 14 & 6 & 2 & 1 \\
\hline 15 & 7 & 2 & 1 \\
\hline 16 & 8 & 2 & 1 \\
\hline 17 & 1 & 1 & 2 \\
\hline 18 & 2 & 1 & 2 \\
\hline 19 & 3 & 1 & 2 \\
\hline 20 & 4 & 1 & 2 \\
\hline 21 & 5 & 1 & 2 \\
\hline 22 & 6 & 1 & 2 \\
\hline 23 & 7 & 1 & 2 \\
\hline 24 & 8 & 1 & 2 \\
\hline
\end{tabular}

\begin{tabular}{|c|c|c|c|}
\hline Configuration & $\mathbf{A}$ & B & $\mathbf{C}$ \\
\hline 25 & 1 & 2 & 2 \\
\hline 26 & 2 & 2 & 2 \\
\hline 27 & 3 & 2 & 2 \\
\hline 28 & 4 & 2 & 2 \\
\hline 29 & 5 & 2 & 2 \\
\hline 30 & 6 & 2 & 2 \\
\hline 31 & 7 & 2 & 2 \\
\hline 32 & 8 & 2 & 2 \\
\hline 33 & 1 & 1 & 3 \\
\hline 34 & 2 & 1 & 3 \\
\hline 35 & 3 & 1 & 3 \\
\hline 36 & 4 & 1 & 3 \\
\hline 37 & 5 & 1 & 3 \\
\hline 38 & 6 & 1 & 3 \\
\hline 39 & 7 & 1 & 3 \\
\hline 40 & 8 & 1 & 3 \\
\hline 41 & 1 & 2 & 3 \\
\hline 42 & 2 & 2 & 3 \\
\hline 43 & 3 & 2 & 3 \\
\hline 44 & 4 & 2 & 3 \\
\hline 45 & 5 & 2 & 3 \\
\hline 46 & 6 & 2 & 3 \\
\hline 47 & 7 & 2 & 3 \\
\hline 48 & 8 & 2 & 3 \\
\hline
\end{tabular}


Table 4.3: Whole-plot experimental configurations for the modeling experiment.

\begin{tabular}{|c|c|c|c|}
\hline Configuration & D & $\mathbf{E}$ & $\mathbf{F}$ \\
\hline 1 & 1 & 1 & 1 \\
\hline 2 & 2 & 1 & 1 \\
\hline 3 & 1 & 3 & 1 \\
\hline 4 & 2 & 3 & 1 \\
\hline 5 & 1 & 5 & 1 \\
\hline 6 & 2 & 5 & 1 \\
\hline 7 & 1 & 1 & 2 \\
\hline 8 & 2 & 1 & 2 \\
\hline 9 & 1 & 3 & 2 \\
\hline 10 & 2 & 3 & 2 \\
\hline 11 & 1 & 5 & 2 \\
\hline 12 & 2 & 5 & 2 \\
\hline 13 & 1 & 2 & 1 \\
\hline 14 & 2 & 2 & 1 \\
\hline 15 & 1 & 4 & 1 \\
\hline 16 & 2 & 4 & 1 \\
\hline 17 & 1 & 6 & 1 \\
\hline 18 & 2 & 6 & 1 \\
\hline 19 & 1 & 2 & 2 \\
\hline 20 & 2 & 2 & 2 \\
\hline 21 & 1 & 4 & 2 \\
\hline 22 & 2 & 4 & 2 \\
\hline 23 & 1 & 6 & 2 \\
\hline 24 & 2 & 6 & 2 \\
\hline
\end{tabular}


Table 4.4: Split-plot experimental configurations for the monitoring experiment.

\begin{tabular}{|c|c|c|c|}
\hline Configuration & A & B & C \\
\hline 1 & 1 & 1 & 1 \\
\hline 2 & 2 & 1 & 1 \\
\hline 3 & 3 & 1 & 1 \\
\hline 4 & 4 & 1 & 1 \\
\hline 5 & 5 & 1 & 1 \\
\hline 6 & 6 & 1 & 1 \\
\hline 7 & 7 & 1 & 1 \\
\hline 8 & 8 & 1 & 1 \\
\hline 9 & 9 & 1 & 1 \\
\hline 10 & 10 & 1 & 1 \\
\hline 11 & 11 & 1 & 1 \\
\hline 12 & 12 & 1 & 1 \\
\hline 13 & 1 & 2 & 1 \\
\hline 14 & 2 & 2 & 1 \\
\hline 15 & 3 & 2 & 1 \\
\hline 16 & 4 & 2 & 1 \\
\hline 17 & 5 & 2 & 1 \\
\hline 18 & 6 & 2 & 1 \\
\hline 19 & 7 & 2 & 1 \\
\hline 20 & 8 & 2 & 1 \\
\hline 21 & 9 & 2 & 1 \\
\hline 22 & 10 & 2 & 1 \\
\hline 23 & 11 & 2 & 1 \\
\hline 24 & 12 & 2 & 1 \\
\hline & & &
\end{tabular}

\begin{tabular}{|c|c|c|c|}
\hline Configuration & A & B & C \\
\hline 25 & 1 & 1 & 2 \\
\hline 26 & 2 & 1 & 2 \\
\hline 27 & 3 & 1 & 2 \\
\hline 28 & 4 & 1 & 2 \\
\hline 29 & 5 & 1 & 2 \\
\hline 30 & 6 & 1 & 2 \\
\hline 31 & 7 & 1 & 2 \\
\hline 32 & 8 & 1 & 2 \\
\hline 33 & 9 & 1 & 2 \\
\hline 34 & 10 & 1 & 2 \\
\hline 35 & 11 & 1 & 2 \\
\hline 36 & 12 & 1 & 2 \\
\hline 37 & 1 & 2 & 2 \\
\hline 38 & 2 & 2 & 2 \\
\hline 39 & 3 & 2 & 2 \\
\hline 40 & 4 & 2 & 2 \\
\hline 41 & 5 & 2 & 2 \\
\hline 42 & 6 & 2 & 2 \\
\hline 43 & 7 & 2 & 2 \\
\hline 44 & 8 & 2 & 2 \\
\hline 45 & 9 & 2 & 2 \\
\hline 46 & 10 & 2 & 2 \\
\hline 47 & 11 & 2 & 2 \\
\hline 48 & 12 & 2 & 2 \\
\hline & & & \\
\hline
\end{tabular}

\begin{tabular}{|c|c|c|c|}
\hline Configuration & A & B & C \\
\hline 49 & 1 & 1 & 3 \\
\hline 50 & 2 & 1 & 3 \\
\hline 51 & 3 & 1 & 3 \\
\hline 52 & 4 & 1 & 3 \\
\hline 53 & 5 & 1 & 3 \\
\hline 54 & 6 & 1 & 3 \\
\hline 55 & 7 & 1 & 3 \\
\hline 56 & 8 & 1 & 3 \\
\hline 57 & 9 & 1 & 3 \\
\hline 58 & 10 & 1 & 3 \\
\hline 59 & 11 & 1 & 3 \\
\hline 60 & 12 & 1 & 3 \\
\hline 61 & 1 & 2 & 3 \\
\hline 62 & 2 & 2 & 3 \\
\hline 63 & 3 & 2 & 3 \\
\hline 64 & 4 & 2 & 3 \\
\hline 65 & 5 & 2 & 3 \\
\hline 66 & 6 & 2 & 3 \\
\hline 67 & 7 & 2 & 3 \\
\hline 68 & 8 & 2 & 3 \\
\hline 69 & 9 & 2 & 3 \\
\hline 70 & 10 & 2 & 3 \\
\hline 71 & 11 & 2 & 3 \\
\hline 72 & 12 & 2 & 3 \\
\hline
\end{tabular}


Table 4.5: Test execution matrix for the modeling and monitoring experiments.

\begin{tabular}{|c|c|c|c|c|c|c|c|c|c|c|c|}
\hline \multicolumn{3}{|c|}{ Round 1} & \multicolumn{3}{|c|}{ Round 2} & \multicolumn{3}{|c|}{ Round 3} & \multicolumn{3}{|c|}{ Round 4} \\
\hline Block & Run & eNB Config & Block & Run & eNB Config & Block & Run & eNB Config & Block & Run & eNB Config \\
\hline \multirow[t]{6}{*}{1} & \multicolumn{2}{|c|}{ Monitoring Test - UE \#1 } & \multirow[t]{6}{*}{1} & \multirow{2}{*}{\multicolumn{2}{|c|}{$\begin{array}{c}\text { Monitoring Test - UE \#4 } \\
\text { UE \#2 }\end{array}$}} & \multirow[t]{6}{*}{1} & \multirow{2}{*}{\multicolumn{2}{|c|}{$\frac{\text { Monitoring Test - UE \#3 }}{\text { UE \#3 }}$}} & \multirow[t]{6}{*}{1} & \multirow{2}{*}{\multicolumn{2}{|c|}{$\begin{array}{c}\text { Monitoring Test - UE \#2 } \\
\text { UE \#4 }\end{array}$}} \\
\hline & \multicolumn{2}{|c|}{\begin{tabular}{|c|} 
UE \#1 \\
\end{tabular}} & & & & & & & & & \\
\hline & 1 & 5 & & 1 & 20 & & 1 & 3 & & 1 & 23 \\
\hline & 2 & 2 & & 2 & 19 & & 2 & 5 & & 2 & 16 \\
\hline & 3 & 6 & & 3 & 24 & & 3 & 9 & & 3 & 19 \\
\hline & 4 & 4 & & 4 & 14 & & 4 & 11 & & 4 & 17 \\
\hline \multirow{7}{*}{2} & \multirow{2}{*}{\multicolumn{2}{|c|}{ Monitoring Test - UE \#2 }} & \multirow{7}{*}{2} & \multirow{2}{*}{\multicolumn{2}{|c|}{ Monitoring Test - UE \#1 }} & \multirow{7}{*}{2} & \multirow{3}{*}{\multicolumn{2}{|c|}{ Monitoring Test - UE \#4 }} & \multirow{7}{*}{2} & \multirow{3}{*}{\multicolumn{2}{|c|}{ Monitoring Test - UE \#3 }} \\
\hline & & & & & & & & & & & \\
\hline & \multicolumn{2}{|c|}{\begin{tabular}{|c|} 
UE \#1 \\
\end{tabular}} & & \multicolumn{2}{|c|}{ UE \#2 } & & UE \#3 & & & & \\
\hline & 5 & 9 & & 5 & 17 & & 5 & 7 & & 5 & 24 \\
\hline & 6 & 8 & & 6 & 21 & & 6 & 4 & & 6 & 15 \\
\hline & 7 & 12 & & 7 & 18 & & 7 & 2 & & 7 & 21 \\
\hline & 8 & 11 & & 8 & 16 & & 8 & 8 & & 8 & 13 \\
\hline \multirow[t]{6}{*}{3} & \multirow{2}{*}{\multicolumn{2}{|c|}{\begin{tabular}{|c|} 
Monitoring Test - UE \#3 \\
UE \#1
\end{tabular}}} & \multirow[t]{6}{*}{3} & Monitc & g Test - UE \#2 & \multirow[t]{6}{*}{3} & Monit & lg Test - UE \#1 & \multirow[t]{6}{*}{3} & Monit & 1g Test - UE \#4 \\
\hline & & & & & UE \#2 & & & UE \#3 & & & UE \#4 \\
\hline & 9 & 7 & & 9 & 15 & & 9 & 1 & & 9 & 14 \\
\hline & 10 & 1 & & 10 & 22 & & 10 & 10 & & 10 & 20 \\
\hline & 11 & 10 & & 11 & 13 & & 11 & 12 & & 11 & 18 \\
\hline & 12 & 3 & & 12 & 23 & & 12 & 6 & & 12 & 22 \\
\hline
\end{tabular}

\subsection{Test Execution}

The test execution matrix for the modeling and monitoring experiments is given in Table 4.5. As outlined in the previous section, the modeling experiment consisted of four rounds of testing, where the test order of the eNB configurations (whole-plots) and non-eNB configurations (split-plots) was randomized to mitigate effects from uncontrolled factors.

Four copies of same UE model were tested across the experiment, where a different UE was used for each round of the modeling experiment. As shown in Table 4.5, the monitoring design was tested at the start of each block with a different UE, where the UE was changed in a repeating order: 1234, 1234, etc. 


\section{Chapter 5}

\section{Statistical Analysis}

This chapter presents a statistical analysis of the measured PUSCH EIRP per PRB data collected in the closed-loop power control (CLPC) study. For statistical analysis the CLPC study is considered to be two experiments conducted in parallel, one called the modeling experiment and the other called the monitoring experiment. The data collected in the two experiments are referred to, respectively, as modeling data and monitoring data.

Chapter 5 has five sections. Section 5.1 reviews from Chapter 2 the purposes and basic features of the two CLPC experiments. Sections 5.2 and 5.3 present statistical analyses of the data from the monitoring experiment and the modeling experiment, respectively. Section 5.4 makes an exploratory analysis of elevated variability found within the modeling data at High crosstalk. This chapter's statistical conclusions are presented in Section 5.4 and then summarized in the broader engineering setting of Section 7.1.

\subsection{Basics of the two experiments}

The modeling experiment involves a distributional response, measured PUSCH EIRP per PRB, represented by the 99 centiles of the distribution. The six factors in this experiment are shown in Table 2.1 with their chosen levels. Every combination of factor levels is replicated twice according to the design described in Chapter 4. The goal of the modeling experiment is to construct descriptive model(s) of measured PUSCH EIRP per PRB for the quantitative factors $A$ and $E^{1}$ over continuous ranges represented by the eight levels of factor $A$ and six levels of factor $E$. Factors $B, C, D$, and $F$ are included in the modeling experiment as categorical variables to discover their effects, main and interaction, on the constructed models of measured PUSCH EIRP per PRB.

The primary purpose of the monitoring experiment, which was conducted in parallel with the modeling experiment, is to monitor the measurement testbed's long-term stability and short-term variability. For this purpose the scope of the data collected in the monitoring experiment is limited and replicates were collected at a higher rate-14 replicates in all—over the nearly two months of data collection. The response in this experiment is measured PUSCH EIRP per $\mathrm{PRB}$, represented as in the modeling experiment by the 99 centiles of its distribution. The monitoring experiment had three active factors $A, B$, and $C$, with factors $D, E$, and $F$ fixed to just one level as shown in Table 2.1. Factors $D, E$, and $F$ specify eNB settings. Fixing these factors each to a single level allowed the data in the monitoring experiment to be collected relatively rapidly with no need to reset the eNB.

Both monitoring and modeling data sets were examined for anomalous outliers. The top row of Figure 5.1, displaying the median and the 90th centile of power (measured PUSCH EIRP per PRB ) versus applied attenuation for the full modeling experiment data set, shows outlying data points clearly outside the range of the majority of the data. Further investigation revealed that all outlying points are associated with cases where the number of active PRBs is less than 1,000. For modeling experiment data where the number of active PRBs is greater than 1,000 , the number of active

\footnotetext{
${ }^{1}$ Factor $E$, the nominal power $P_{0}$, is a UE-specific (optionally cell-specific) parameter in UE fractional power control (FPC). Factor $F$, the power fraction $\alpha$, called more fully the fractional path-loss compensation factor, is another FPC parameter. Engineering aspects of the experiments are detailed in Chapter 6.
} 

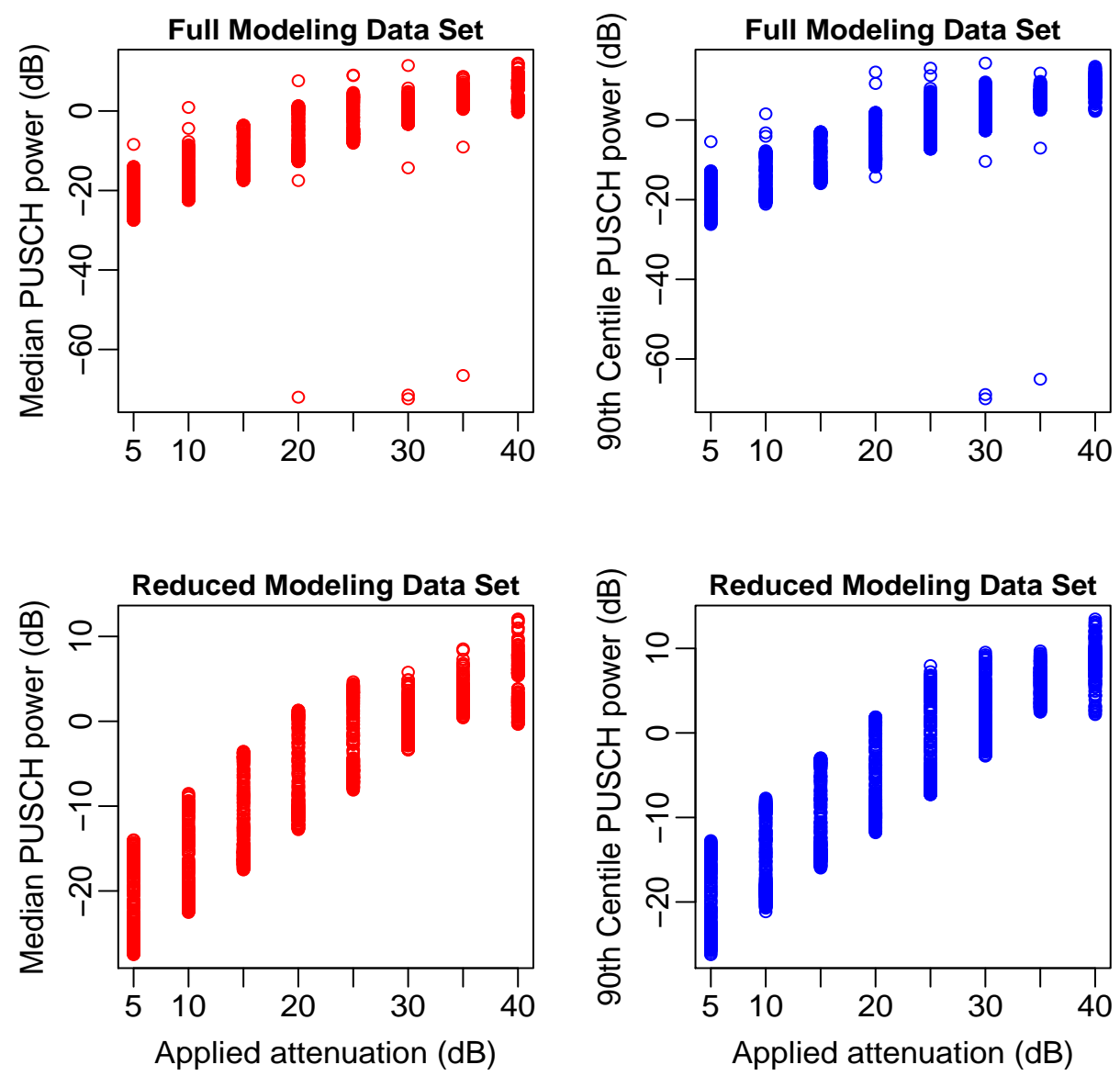

Figure 5.1: Median and 90th centile of power (measured PUSCH EIRP per PRB) versus applied attenuation for the full modeling experiment data set (top row) and for the modeling experiment data set where the number of active PRBs is greater than 1,000 (bottom row).

PRBs ranges from 5,036 to 135,419, so a small number of active PRBs is taken to be an indicator of poor data quality ${ }^{2}$. The bottom row of the plots in Figure 5.1 displays the modeling experiment data where the number of active PRBs is greater than 1,000. The analyses reported in Chapters 5 and for both the monitoring and the modeling experiments are based on data sets where the number of active PRBs is greater than 1,000 . The number of excluded cases is $1.4 \%$ for the monitoring experiment and $1.2 \%$ for the modeling experiment.

The fourteen replicate sets of runs made in the monitoring experiment offer an opportunity to make some limited study of phenomena that could not be addressed by just the two replicates in the modeling experiment with its larger number of active factors and factor combinations. While the monitoring experiment's primary purpose is to monitor

\footnotetext{
${ }^{2}$ Due to the rare and intermittent nature of test runs with poor data quality, identifying the root cause would require significant additional testing and troubleshooting. Because this issue only occurred in a few isolated test cases, investigation into the cause of poor data quality in these instances was not pursued.
} 
the measurement testbed, it is designed also for two other purposes. The first of these secondary purposes is to explore negative power headroom in fuller detail than the modeling experiment's design allows. To accomplish this, additional high levels of applied attenuation are included in the experiment design, for a total of twelve levels in the monitoring experiment versus eight in the modeling experiment. The other secondary purpose is to see how much, if any, variability in the measured PUSCH EIRP per PRB response is contributed by different copies of the DUT UE. Four copies (varying only by serial number) of a moderately expensive UE, purchased from different vendors, were used in the two experiments. In the four rounds of the modeling experiment, these handsets were used, one per round, in order 1,2,3,4, so half of the replicate run pairs used handsets 1 and 3 while the other half used handsets 2 and 4 . This arrangement confounds variation due to UE DUT with variation from other unidentified sources. In the fourteen replicates in the monitoring experiment, the handsets were used in the order 1,2,3,4,1,2,3,4,1,2,3,4,2,3. For example, four runs were made with handset 3 with identical combinations of factor settings. This replication in the monitoring data allows an estimate of handset-contributed variability.

\subsection{Analysis of the monitoring data}

Statistical analyses of the data collected in the monitoring experiment are presented in this section. The monitoring data are used first to check the performance of the testbed. This check shows, in short, that the testbed was stable over the set of fourteen times where data were collected. This check also shows the presence of an unidentified and unexpected phenomenon/variable operating at High crosstalk. The unidentified variable has so large an effect, relative to those of the established factors in this study, that modeling without accounting for its presence is potentially misleading. The High crosstalk data are therefore excluded from our subsequent modeling efforts, limiting the scope of these models to Low crosstalk. Next, the effects of handset and offered load in the data are analyzed, treating the effects as random and fixed, respectively. Using the conclusions of the various analyses, models of centiles of measured PUSCH EIRP per PRB are developed based on the monitoring data. Because factor $E$ is fixed to the single value $-85 \mathrm{~dB}$ in the monitoring data, the only continuous independent variable in these models is factor $A$, applied attenuation. The centile models from the monitoring data are therefore simple and limited in scope; they are, though, a useful guide to the development of descriptive centile models for the modeling data in the next section.

\subsubsection{Testbed stability}

The performance of the measurement testbed over nearly two months of operation can be seen in Figs. 5.2-5.4. These three figures show the monitoring data collected at three representative levels, $10,20,30 \mathrm{~dB}$, of factor $A$. Each figure contains six timeplots of measured PUSCH EIRP per PRB collected at fourteen times over the two-month period of the CLPC study. The dark blue lines in each timeplot are 50th centiles of measured PUSCH EIRP per PRB; the light blue lines are the 5th and 95th centiles. The six timeplots in each figure correspond to the six combinations of levels of factors $B$ and $C$. Connecting lines have been added to the plotted points in each figure to aid visual assessment of the changes in the distribution of measured PUSCH EIRP per PRB.

The timeplots in Figs. 5.2-5.4 are examined for longer-term stability of the testbed. Stability refers to general trends in the testbed output; for example, measured powers that rise on average over time. The timeplots suggest no such longer term trend, and formal statistical analysis ${ }^{3}$ confirms this. The testbed remained stable over its fourteen time points of use in two months of operation.

\subsubsection{Unknown phenomenon at High crosstalk}

The right-hand timeplots in Figs. 5.2-5.4 show noticeable shorter-term variability in measured PUSCH EIRP per PRB. These right-hand plots are the High crosstalk cases. Each of the eighteen timeplots in Figs. 5.2-5.4 have fixed levels of factors $A, B$, and $C$; also, of course, the levels of factors $D, E$, and $F$ are fixed. In each timeplot all that varies among the fourteen measurements are the time of measurement and the particular handset used; recall that the four

\footnotetext{
${ }^{3}$ Straight-line regressions were done on different subsequences of the 5th, 50th, and 95th centiles in each of Figs. 5.2-5.4. No regression yielded significant evidence for a trend with non-zero slope.
} 
handsets were used in the order 1,2,3,4,1,2,3,4,1,2,3,4,2,3. To determine whether the handsets are the source of the variability seen in Figs. 5.2-5.4, the results for measured PUSCH EIRP per PRB are rearranged in Figs. 5.5-5.7 so that the results are grouped by handset. The left-hand plots in Figs. 5.5-5.7 show almost no variability due to handset at Low crosstalk. In the right-hand timeplots with High crosstalk, the variability with the same handset and all factors held constant is large. In some cases the 95th centile of measured PUSCH EIRP per PRB is actually less than the 5th centile at a different instance with the same handset. For examples, see handset 4 in the top right-hand timeplot in Fig. 5.5 or handset 3 in the bottom right-hand timeplot in Fig. 5.6.

The nature and origin of the unknown variable operating at High crosstalk are unclear. The variable must originate either from the testbed measurement system, from the LTE system under test, or from a source external to the testbed. A source external to the testbed is highly unlikely; it would have to somehow operate in coordination with just the High crosstalk runs or have some extraordinary interactive effect with crosstalk. Also, repeated careful inspection of the testbed measurement system and the data from the Low crosstalk runs together argue against the measurement system as the source of this unknown variable. The unknown variable evidenced at High crosstalk in Figs. 5.5-5.7 most likely originates with the LTE system. One can speculate about some type of system memory, a hysteresis effect, or evolution to a multi-modal steady state at High crosstalk. The testbed and experiment design did not anticipate this phenomenon and were not prepared to identify its origin or nature. Lacking this information, modeling measured PUSCH EIRP per PRB at High crosstalk is questionable, and all modeling for the CLPC study is restricted to just the Low crosstalk data. The detection of the High crosstalk phenomenon, though, is an interesting and potentially important result of the study.
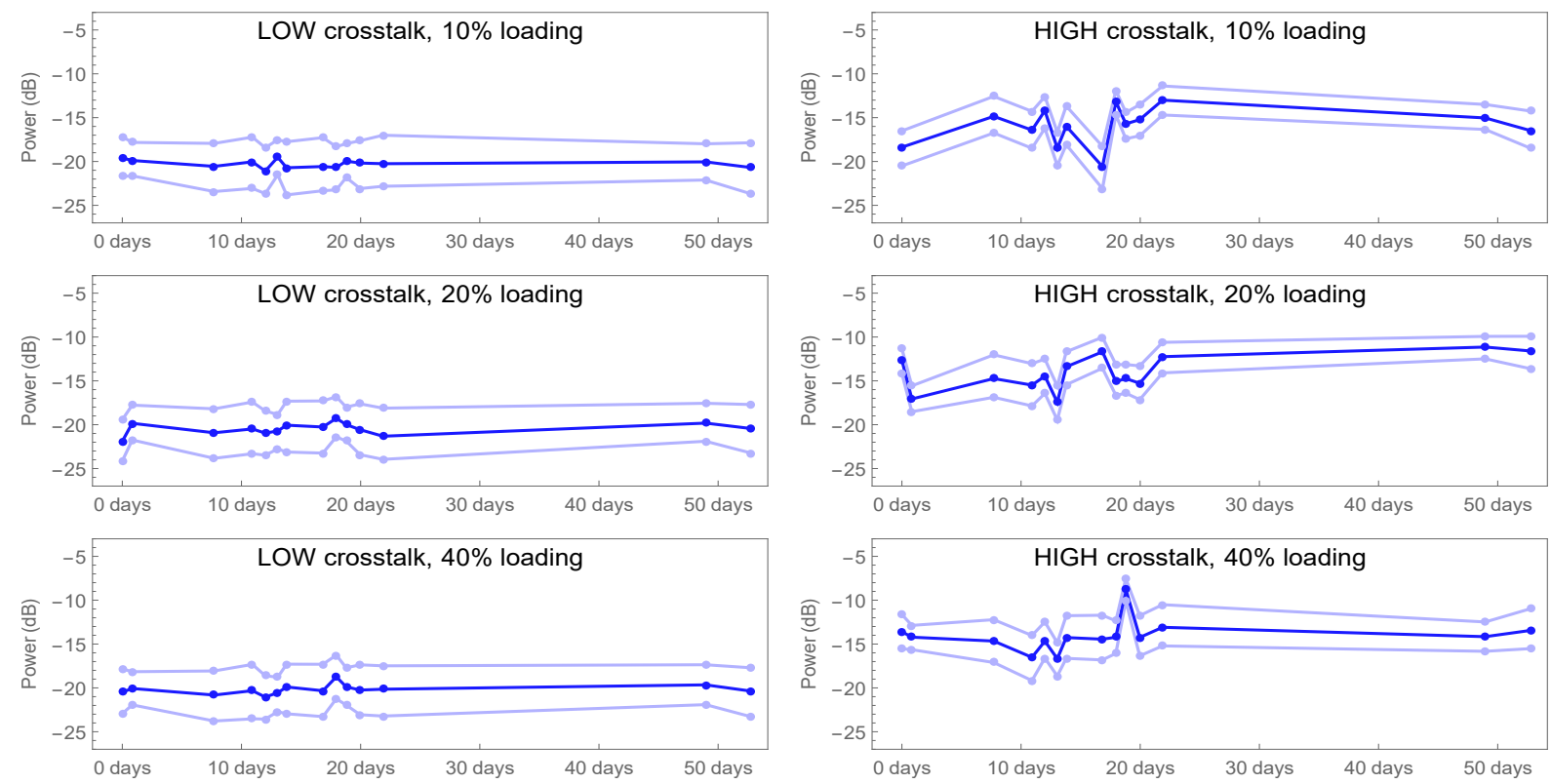

Figure 5.2: Power (measured PUSCH EIRP per PRB) at $10 \mathrm{~dB}$ applied attenuation at fourteen time points over the two months of the monitoring experiment. The six timeplots correspond to the six combinations of factors $B$ and $C$, crosstalk and offered load. The dark blue points in each plot are medians of the measured PUSCH EIRP per PRB distribution; the light blue points are the 5th and 95th centiles. The plotted points are connected by lines to aid visual assessment of changes in power. 

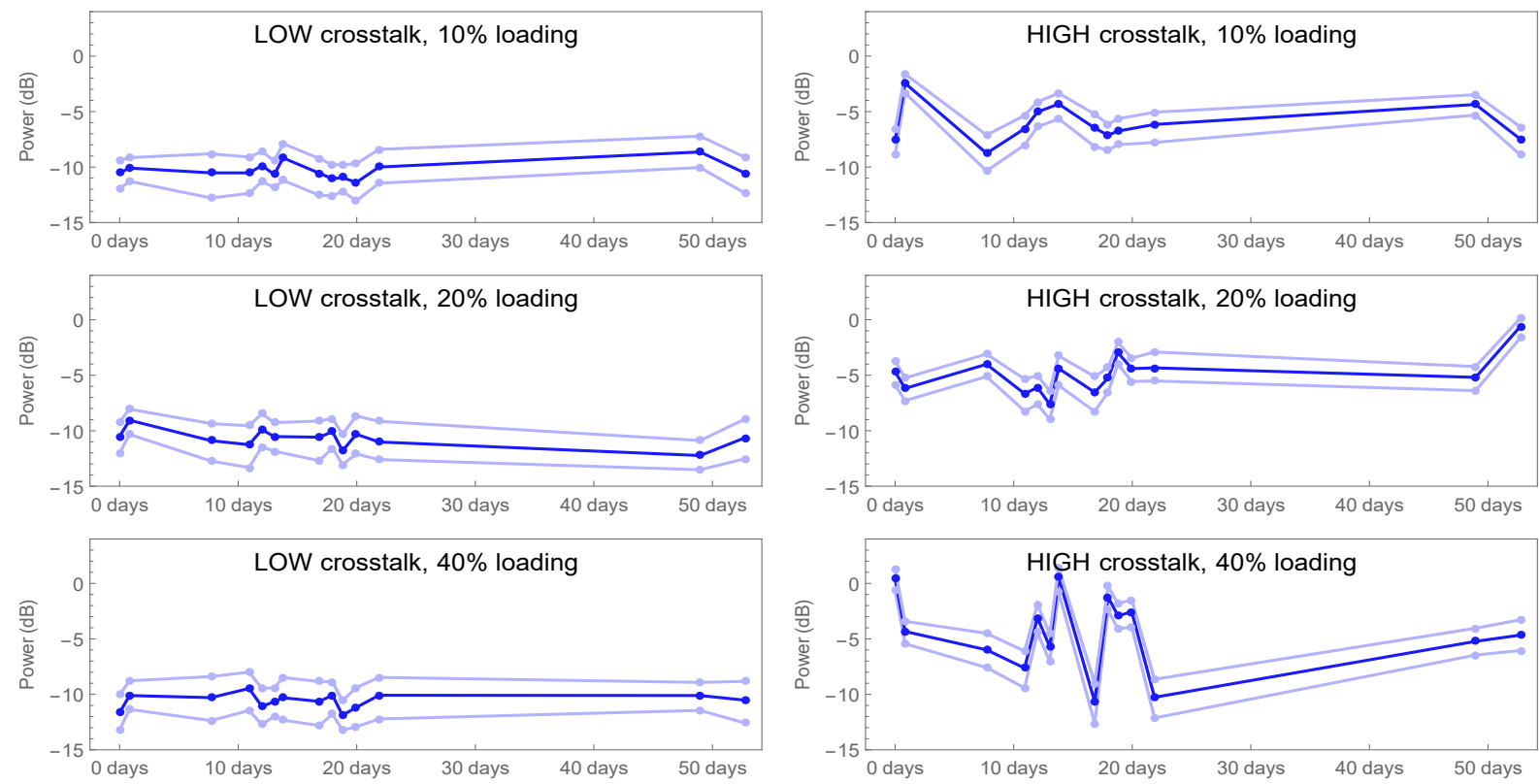

Figure 5.3: Power (measured PUSCH EIRP per PRB) at $20 \mathrm{~dB}$ applied attenuation at fourteen time points over the two months of the monitoring experiment. The six timeplots correspond to the six combinations of factors $B$ and $C$, crosstalk and offered load. The dark blue points in each plot are medians of the measured PUSCH EIRP per PRB distribution; the light blue points are the 5th and 95 th centiles. The plotted points are connected by lines to aid visual assessment of changes in power.
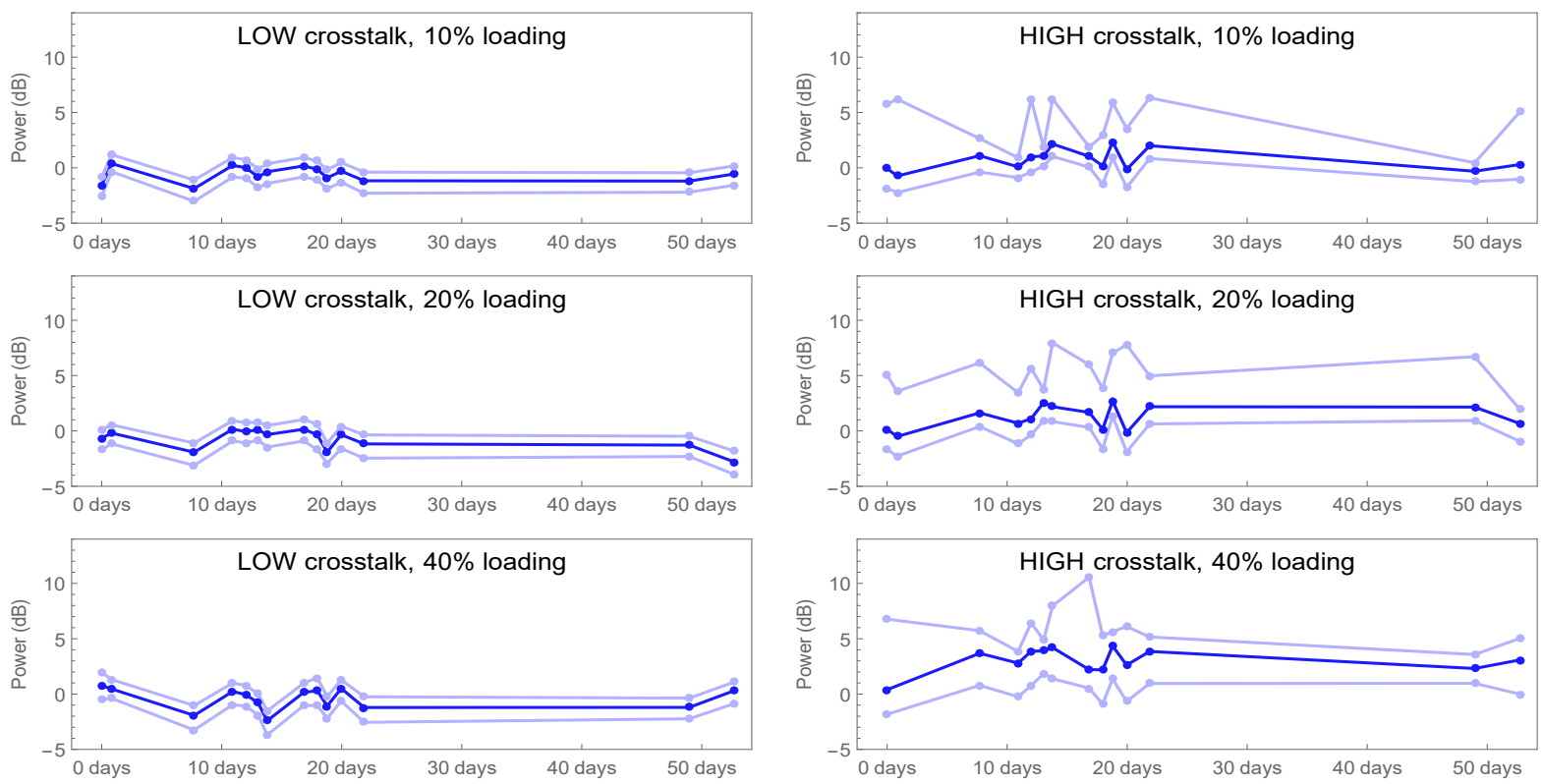

Figure 5.4: Power (measured PUSCH EIRP per PRB) at $30 \mathrm{~dB}$ applied attenuation at fourteen time points over the two months of the monitoring experiment. The six timeplots correspond to the six combinations of factors $B$ and $C$, crosstalk and offered load. The dark blue points in each plot are medians of the measured PUSCH EIRP per PRB distribution; the light blue points are the 5th and 95th centiles. The plotted points are connected by lines to aid visual assessment of changes in power. 

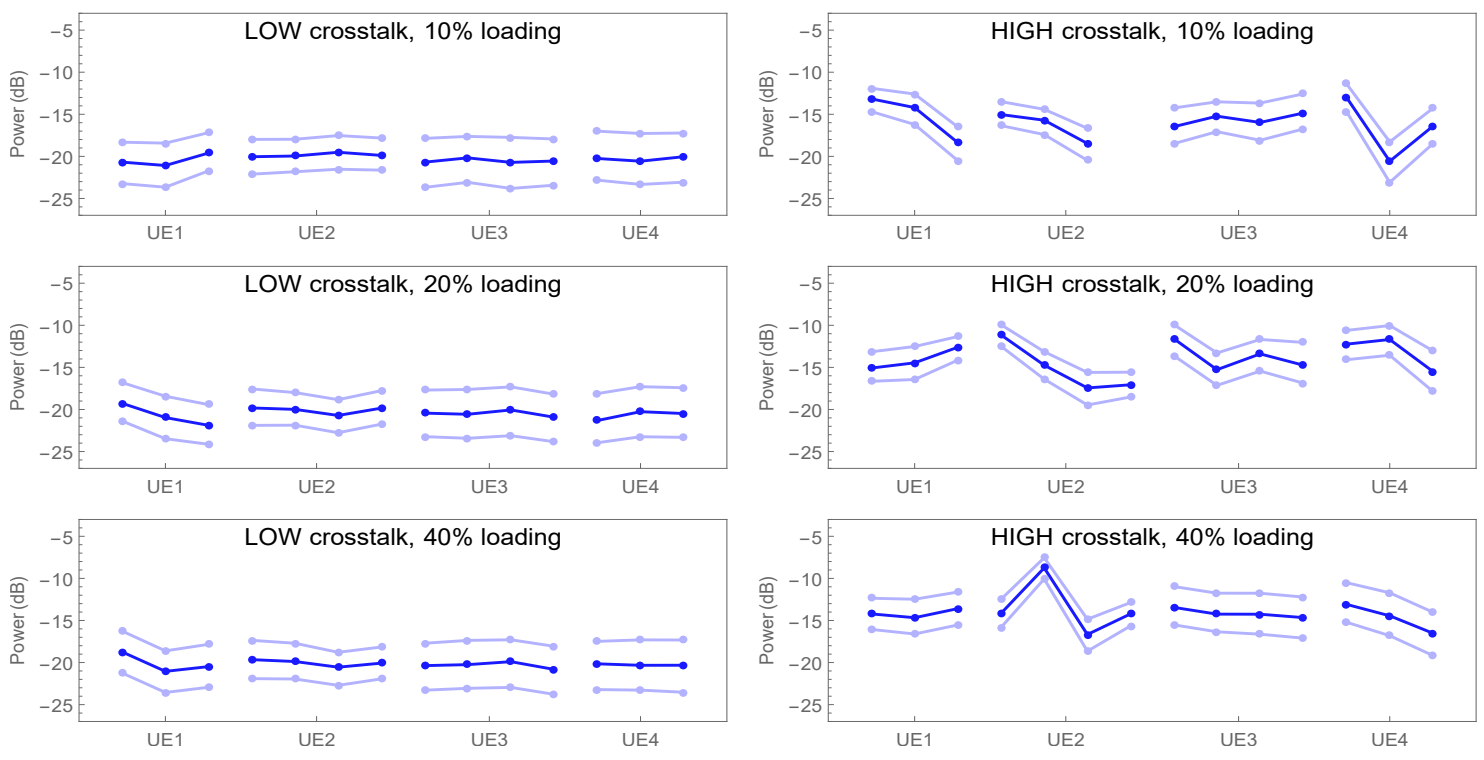

Figure 5.5: Power (Measured PUSCH EIRP per PRB) at $10 \mathrm{~dB}$ applied attenuation in the monitoring experiment grouped by UE DUT. The six time plots correspond to the six combinations of factors $B$ and $C$, crosstalk and offered load. The dark blue points in each plot are medians of the measured PUSCH EIRP per PRB distribution; the light blue points are the 5 th and 95 th centiles.
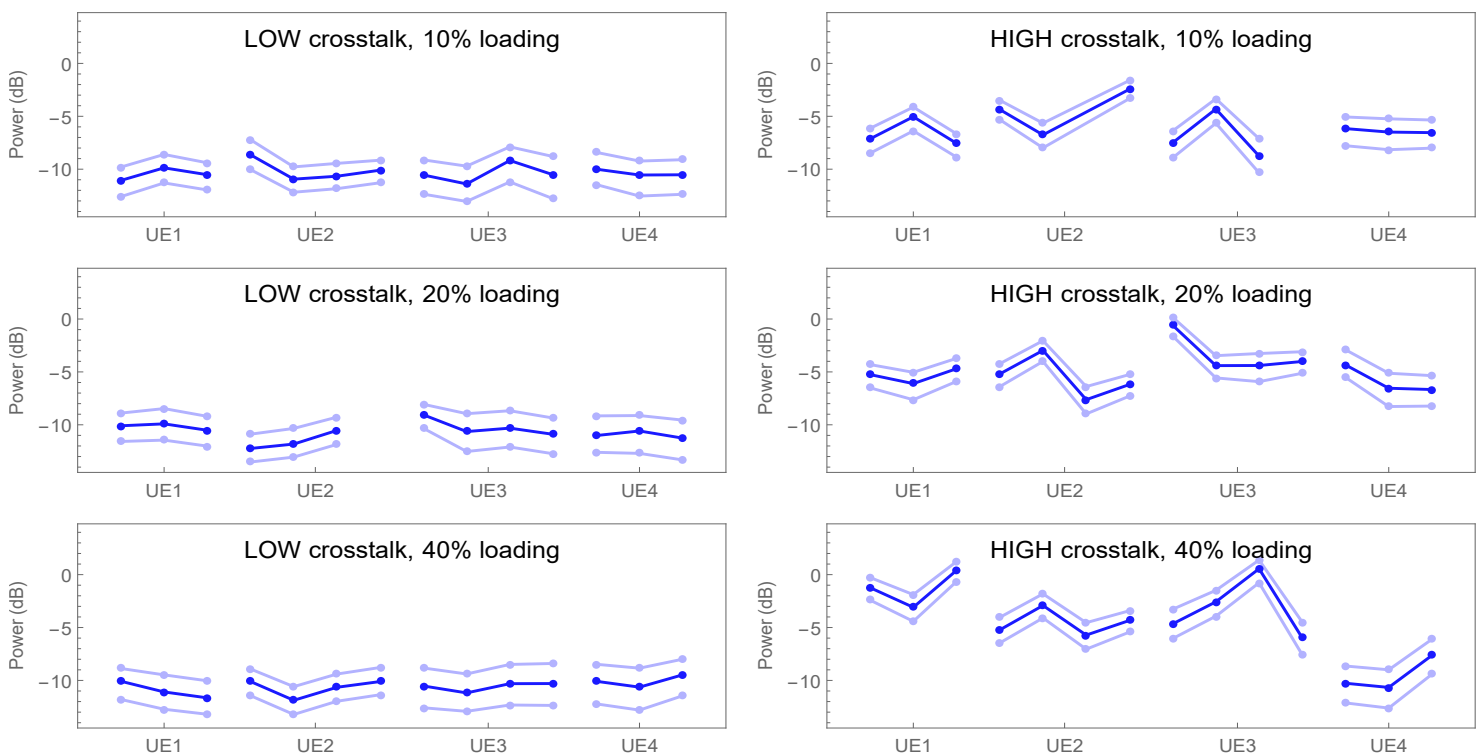

Figure 5.6: Power (measured PUSCH EIRP per PRB) at $20 \mathrm{~dB}$ applied attenuation in the monitoring experiment grouped by UE DUT. The six time plots correspond to the six combinations of factors $B$ and $C$, crosstalk and offered load. The dark blue points in each plot are medians of the measured PUSCH EIRP per PRB distribution; the light blue points are the 5 th and 95 th centiles. 

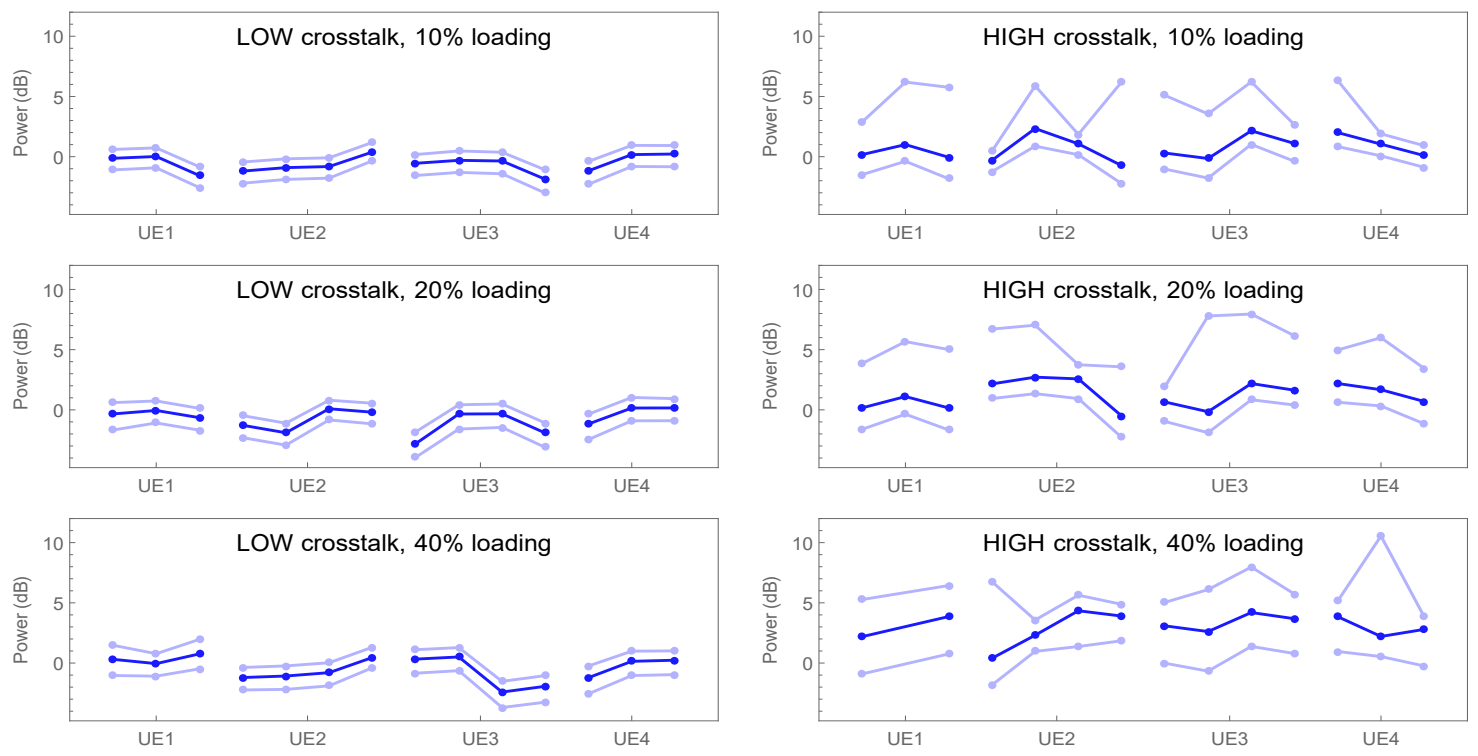

Figure 5.7: Power (measured PUSCH EIRP per PRB) at $30 \mathrm{~dB}$ applied attenuation in the monitoring experiment grouped by UE DUT. The six time plots correspond to the six combinations of factors $B$ and $C$, crosstalk and offered load. The dark blue points in each plot are medians of the measured PUSCH EIRP per PRB distribution; the light blue points are the 5th and 95th centiles.

\subsubsection{Handset-contributed variability}

The differences seen among different handsets in Figs. 5.5-5.7 suggest that handset-contributed variability in measured PUSCH EIRP per PRB is small or non-existent. An analysis of covariance (ANCOVA) ${ }^{4}$ model,

$$
Y=\beta_{0}+\beta_{1} A+\mathrm{UE}+\varepsilon
$$

is used to quantitatively assess the contribution of handset to variability. In the model, $Y$ is a given power centile, $A$ is applied attenuation, $\beta_{0}$ and $\beta_{1}$ are parameters for the overall level of $Y$ and the linear effect of $A$, UE is UE DUT treated as a zero-mean random effect with standard deviation $\sigma$, and $\varepsilon$ reflects random variation from other sources. The standard distribution, independence, and homoscedasticity assumptions [12] are made for the model, and the model is fitted to just data with $A<30 \mathrm{~dB}$, where the effect of $A$ on measured power is linear. A different instance of the model is fit for each of the six combinations of factor $B$ and $C$ levels, with results for the 50th centile of power in Table 5.1 and for the 90th centile in Table 5.2.

The ANCOVA results in Tables 5.1 and 5.2 show that the estimated standard deviations of handset-contributed variability in the 50th and 90th centiles of measured PUSCH EIRP per PRB are small—around $1 \mathrm{~dB}$ or less—and not discernibly different from zero. The descriptive models of measured PUSCH EIRP per PRB are based on just Low crosstalk; according to Tables 5.1 and 5.2, for these cases the estimated standard deviation is no more than $0.2 \mathrm{~dB}$. These conclusions are based on only one model of UE, and their broader validity is unknown.

\footnotetext{
${ }^{4}$ ANCOVA, or analysis of covariance, is a linear statistical model in which ANOVA is augmented by elements of regression to accommodate the presence of a quantitative variable such as applied attenuation $A$.
} 


\begin{tabular}{l|cc|cc|} 
Offered & \multicolumn{3}{c}{ Crosstalk } \\
load & \multicolumn{2}{c}{$B=$ Low } & $B=$ High \\
\cline { 2 - 6 }$C=10 \%$ & $\mathbf{0 . 2}$ & 0.19 & $\mathbf{0 . 0}$ & 1.00 \\
\cline { 2 - 6 }$C=20 \%$ & $\mathbf{0 . 1}$ & 0.47 & $\mathbf{0 . 9}$ & 0.15 \\
\cline { 2 - 6 }$C=40 \%$ & $\mathbf{0 . 0}$ & 1.00 & $\mathbf{1 . 5}$ & 0.14 \\
\cline { 2 - 6 }$C$ & & & &
\end{tabular}

Table 5.1: Monitoring data ANCOVA modeling of the 50th centile of measured PUSCH EIRP per PRB. Table entries in boldface are estimated standard deviations $\hat{\sigma}$ (in $\mathrm{dB}$ ) of the random contribution of handset to the 50th centile of PUSCH EIRP per PRB variability. Corresponding entries in plainface are the associated p-values for the null hypothesis that $\sigma=0$.

\begin{tabular}{l|cc|cc|} 
Offered & \multicolumn{3}{c}{ Crosstalk } \\
load & \multicolumn{2}{c}{$B=$ Low } & $B=$ High \\
\cline { 2 - 5 }$C=10 \%$ & $\mathbf{0 . 1}$ & 0.29 & $\mathbf{0 . 3}$ & 0.37 \\
\cline { 2 - 5 }$C=20 \%$ & $\mathbf{0 . 0}$ & 1.00 & $\mathbf{0 . 9}$ & 0.15 \\
\cline { 2 - 6 }$C=40 \%$ & $\mathbf{0 . 1}$ & 0.42 & $\mathbf{1 . 3}$ & 0.14 \\
\cline { 2 - 5 }$C$ & & & &
\end{tabular}

Table 5.2: Monitoring data ANCOVA modeling of the 90th centile of measured PUSCH EIRP per PRB. Table entries in boldface are estimated standard deviations $\hat{\sigma}$ (in $\mathrm{dB}$ ) of the random contribution of handset to the 90th centile of PUSCH EIRP per PRB variability. Corresponding entries in plainface are the associated p-values for the null hypothesis that $\sigma=0$.

\subsubsection{Offered load effect}

Offered load $C$ was set to three different levels in the monitoring experiment. The effect of offered load on measured PUSCH EIRP per PRB is quantitatively assessed with the ANCOVA model

$$
Y=\beta_{0}+\beta_{1} C_{1}+\beta_{2} C_{2}+\beta_{3} A+\beta_{4} C_{1} A+\beta_{5} C_{2} A+\varepsilon,
$$

where $Y$ is a given power centile, $A$ is applied attenuation, $\beta_{0}$ and $\beta_{3}$ are parameters for the overall level of $Y$ and the linear effect of $A$, and $\varepsilon$ reflects random variation in $Y$. Main and interaction effects of offered load's three levels are represented in (5.1) through the two indicator variables $C_{1}, C_{2}$ for $C$. The standard ANCOVA distribution, independence, and homoscedasticity assumptions are made, and the model is fit to just data with $A<30 \mathrm{~dB}$, where the effect of $A$ on measured power is linear (in units of $\mathrm{dB}$ ). UE DUT is not included as a variable in (5.1) because its effect on measured PUSCH EIRP per PRB was established earlier to be small or nonexistent. Model (5.1) is fit for Low crosstalk only, so crosstalk does not appear as a variable. 
The ANCOVA results in Tables 5.3 and 5.4 show, in summary, that offered load $C$, either on its own or in interaction with $A$, has no discernible effect on the 50th or 90th centiles of measured PUSCH EIRP per PRB. According to the ANCOVA results on the left in Table 5.3, for example, the main and interaction effects of $C$ on the 50th centile of measured PUSCH EIRP per PRB have p-values of 0.80 and 0.68 , respectively. A p-value below the 0.05 significance level indicates the presence of a discernible effect. These high p-values indicate no evidence for main or interaction effects. The right sides of Tables 5.3 and 5.4 report the effect sizes associated with the three differences among the three levels of offered load. For example, the mean change in the 50th centile of measured power caused by a change in offered load from $20 \%$ to $40 \%$ is estimated to be $0.11 \mathrm{~dB}$, with a standard error of $0.17 \mathrm{~dB}$. The effect of offered load is small and is not discernibly different from zero, consistent with the ANCOVA p-values for offered load on the left side of Table 5.3. The conclusions in Tables 5.3 and 5.4 only apply to offered load at levels of 10, 20, and 40\%. Outside this range, offered load might have important effects not revealed in the monitoring experiment.

\begin{tabular}{|c|c|c|c|c|c|}
\hline Effect & P-value & Difference & $\begin{array}{l}\text { Estimated mean } \\
\text { effect }(\mathrm{dB})\end{array}$ & $\begin{array}{c}\text { Standard } \\
\text { error }(\mathbf{d B})\end{array}$ & P-value \\
\hline$A$ & 0.00 & $Y_{C=20 \%}-Y_{C=10 \%}$ & -0.06 & 0.17 & 0.72 \\
\hline$C$ & 0.80 & $Y_{C=40 \%}-Y_{C=20 \%}$ & 0.11 & 0.17 & 0.51 \\
\hline$C \cdot A$ & 0.68 & $Y_{C=40 \%}-Y_{C=10 \%}$ & 0.05 & 0.17 & 0.77 \\
\hline
\end{tabular}

Table 5.3: Monitoring data ANCOVA modeling of the 50th centile of measured PUSCH EIRP per PRB to assess the effect of offered load.

\begin{tabular}{c|c|} 
Effect & P-value \\
$A$ & 0.00 \\
\cline { 2 - 2 }$C$ & 0.93 \\
\cline { 2 - 2 }$C \cdot A$ & 0.83 \\
\cline { 2 - 2 } &
\end{tabular}

\begin{tabular}{c|ccc|}
\multicolumn{1}{c}{$\begin{array}{c}\text { Estimated mean } \\
\text { Difference }\end{array}$} & \multicolumn{1}{c}{$\begin{array}{c}\text { Standard } \\
\text { error (dB) }\end{array}$} & \multicolumn{1}{c}{ P-value } \\
$Y_{C=20 \%}-Y_{C=10 \%}$ & 0.00 & 0.19 & 0.98 \\
\cline { 2 - 4 }$Y_{C=40 \%}-Y_{C=20 \%}$ & 0.06 & 0.19 & 0.75 \\
\cline { 2 - 4 }$Y_{C=40 \%}-Y_{C=10 \%}$ & 0.06 & 0.19 & 0.73 \\
\cline { 2 - 4 } & &
\end{tabular}

Table 5.4: Monitoring data ANCOVA modeling of the 90th centile of measured PUSCH EIRP per PRB to assess the effect of offered load. 


\subsubsection{Descriptive models}

The monitoring experiment involves three factors, applied attenuation $A$, crosstalk $B$, and offered load $C$, as well as a random contribution due to UE DUT. All four of these elements might have been found to be necessary to modeling the centiles of measured PUSCH EIRP per PRB. Instead, $B$ is removed as a model input since only a single level is considered. Additionally, $C$ and UE were found by the data to have no statistically discernible effects on measured PUSCH EIRP per PRB, and they too are removed as model inputs. Over the domain of the data collected in the monitoring experiment, only $A$ is available as a model input. A continuous, piecewise linear function of $A$ is proposed as a model for each centile $Y$ of measured PUSCH EIRP per PRB (in dB):

$$
Y=\beta_{0}+\beta_{1} A+\left(\beta_{3}-\beta_{1}\right)\left(A-\beta_{2}\right) 1\left(A-\beta_{2}\right),
$$

where $\beta_{0}, \beta_{1}, \beta_{2}$, and $\beta_{3}$ are model parameters, representing, respectively, the $Y$-intercept, the slope before the model changepoint, the changepoint, and the slope after the changepoint. The indicator function $\mathbf{1}(x)$ in (5.2) is one when $x$ is positive and zero otherwise.

Fig. 5.8 shows model (5.2) fitted to the monitoring data for the 50th and 90th centiles of measured PUSCH EIRP per PRB. A standard iterative nonlinear least-squares fitting procedure was used to obtain the model fits. The models are shown together at the bottom in Fig. 5.8 with corresponding models for the 5th and 95th centiles of measured PUSCH EIRP per PRB. The estimated parameters with their standard errors for the four models are presented in Table 5.5. Visually, these models appear qualitatively to be a good fit to the data. Two quantitative measures of fit, the coefficient of determination $\left(R^{2}\right)$ and model RMS error, support the visual assessment. According to the models' $R^{2}$ values in Table 5.5, each model explains at least $99.0 \%$ of the variability in its centile response. A more complicated model with more parameters or extra factors could at best increase $R^{2}$ by only a fraction of a percent and would risk speculatively overfitting the data. The four models' RMS errors in Table 5.5 are around $1 \mathrm{~dB}$. This is close to the minimum RMS error possible given the variation in the measured PUSCH EIRP per PRB centiles at each level of applied attenuation.

The description of measured PUSCH EIRP per PRB offered by the centile models shown in Fig. 5.8 and detailed in Table 5.5 have some notable features:

1. Model (5.2) does not involve factor $C$. Offered load at the levels considered in this study has no discernible effect on the relationship in the monitoring data between centile response and applied attenuation, significantly simplifying this relationship.

2. Only one form of model—continuous, piecewise linear-is needed to model the different centiles of measured PUSCH EIRP per PRB.

3. The measured PUSCH EIRP per PRB centiles have a clear changepoint in their relationship to applied attenuation.

4. The slope to the left of the changepoint is very close to 1 in the centile models (see Table 5.2). This is unexpected because the data from the monitoring experiment were collected with the eNB power fraction set to $\alpha=0.8$.

5. The slope to the right of the changepoint in each the centile model is significantly less than zero. Power decreases above the changepoint are unexpected.

6. The position of the changepoint in the centile models changes with the centile level as shown in Fig. 5.9. Most of the measured PUSCH EIRP per PRB distribution (below its 70th centile) increases coherently (the centiles move in unison) at a near unit rate (approximately $1 \mathrm{~dB}$ of power per $\mathrm{dB}$ of applied attenuation) as the applied attenuation is increased. The increase in measured PUSCH EIRP per PRB continues until the centiles in the lower $70 \%$ of the power distribution each reaches its changepoint between 33 and $34 \mathrm{~dB}$ applied attentuation, where the power stops increasing and begins to slowly fall. The upper tail of the distribution (above the 70th centile) continues to increase with applied attenuation as much as $5 \mathrm{~dB}$ more until its changepoint is reached and the power slowly falls. 
These enumerated features of our descriptive models of measured PUSCH EIRP per PRB are explored from an engineering perspective in Chapter 6. Beyond the immediate insights these centile models offer for understanding measured PUSCH EIRP per PRB in the monitoring data, they also guide the descriptive modeling in the next section of the measured PUSCH EIRP per PRB data collected in the modeling experiment.
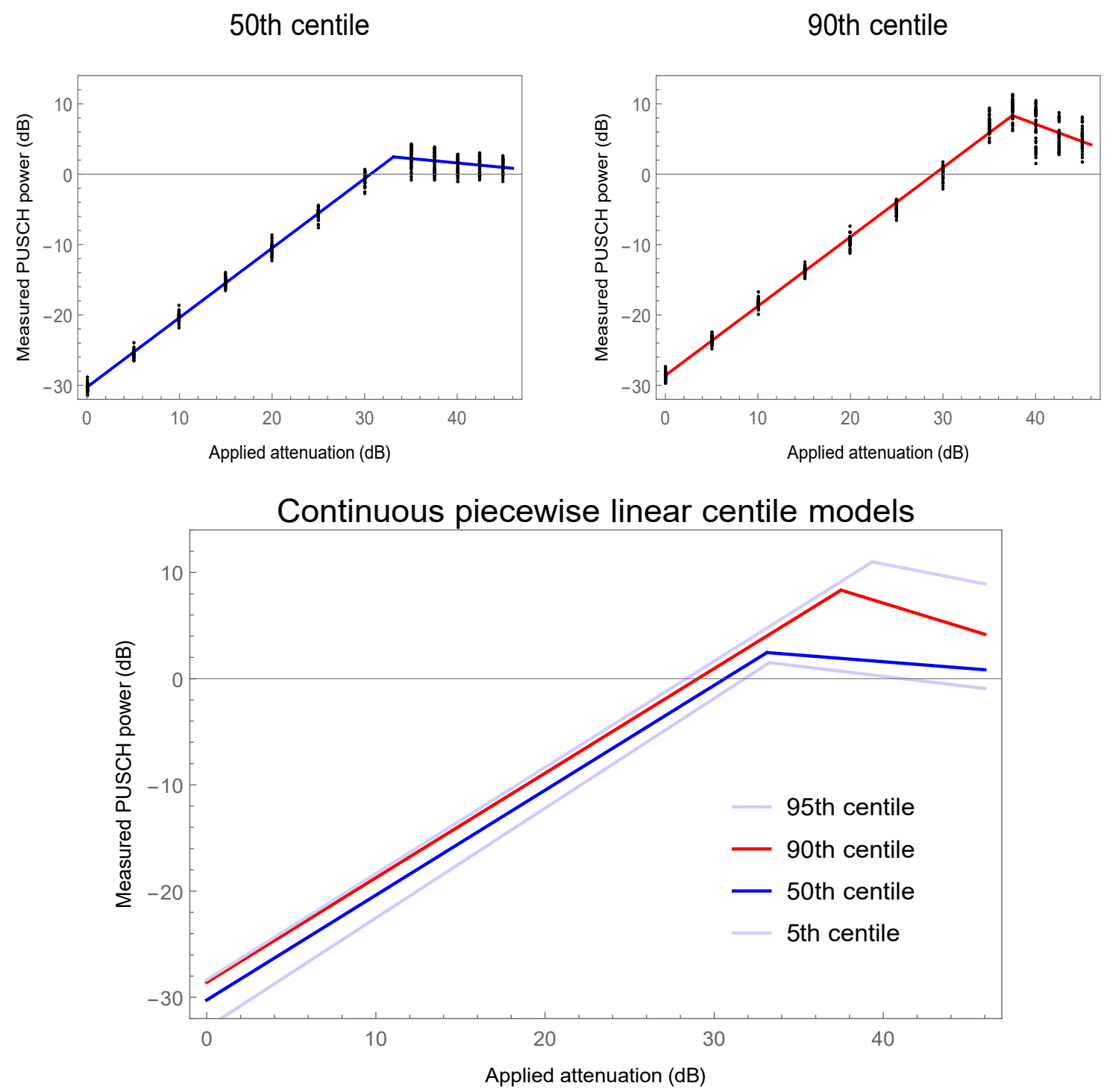

Figure 5.8: Fitted models of 50th and 90th centiles of measured PUSCH EIRP per PRB are shown (top left, top right) with the monitoring data on which these models are based. Fitted models of the 5th, 50th, 90th, and 95th centiles of measured PUSCH EIRP per PRB based on the data collected in the monitoring experiment are compared (bottom). 


\begin{tabular}{cccc}
$\begin{array}{c}\text { Measured PUSCH EIRP } \\
\text { per PRB centile model }\end{array}$ & $\begin{array}{c}\text { Model } \\
\text { parameter }\end{array}$ & $\begin{array}{c}\text { Parameter } \\
\text { estimate }\end{array}$ & $\begin{array}{c}\text { Standard } \\
\text { error }\end{array}$ \\
\hline \hline 5th centile & $\beta_{0}$ & -32.86 & 0.11 \\
$R^{2}=99.6 \%$ & $\beta_{1}$ & 1.033 & 0.006 \\
RMS error $=1.01 \mathrm{~dB}$ & $\beta_{2}$ & 33.26 & 0.16 \\
& $\beta_{3}$ & -0.191 & 0.020 \\
\hline 50th centile & $\beta_{0}$ & -30.26 & 0.10 \\
$R^{2}=99.6 \%$ & $\beta_{1}$ & 0.988 & 0.005 \\
RMS error $=0.92 \mathrm{~dB}$ & $\beta_{2}$ & 33.12 & 0.16 \\
& $\beta_{3}$ & -0.126 & 0.018 \\
\hline 90th centile & $\beta_{0}$ & -28.60 & 0.14 \\
$R^{2}=99.0 \%$ & $\beta_{1}$ & 0.985 & 0.007 \\
RMS error $=1.36 \mathrm{~dB}$ & $\beta_{2}$ & 37.50 & 0.16 \\
& $\beta_{3}$ & -0.489 & 0.039 \\
\hline 95th centile & $\beta_{0}$ & -28.35 & 0.12 \\
$R^{2}=99.2 \%$ & $\beta_{1}$ & 1.000 & 0.005 \\
RMS error $=1.28 \mathrm{~dB}$ & $\beta_{2}$ & 39.35 & 0.19 \\
& $\beta_{3}$ & -0.311 & 0.057 \\
\hline \hline
\end{tabular}

Table 5.5: Continuous, piecewise linear models of select centiles of measured PUSCH EIRP per PRB. Parameter estimates of $\beta_{0}$ and $\beta_{2}$ and their standard errors are in reported in decibels. Parameters $\beta_{1}$ and $\beta_{3}$ are slopes; their estimates and standard errors are dimensionless.

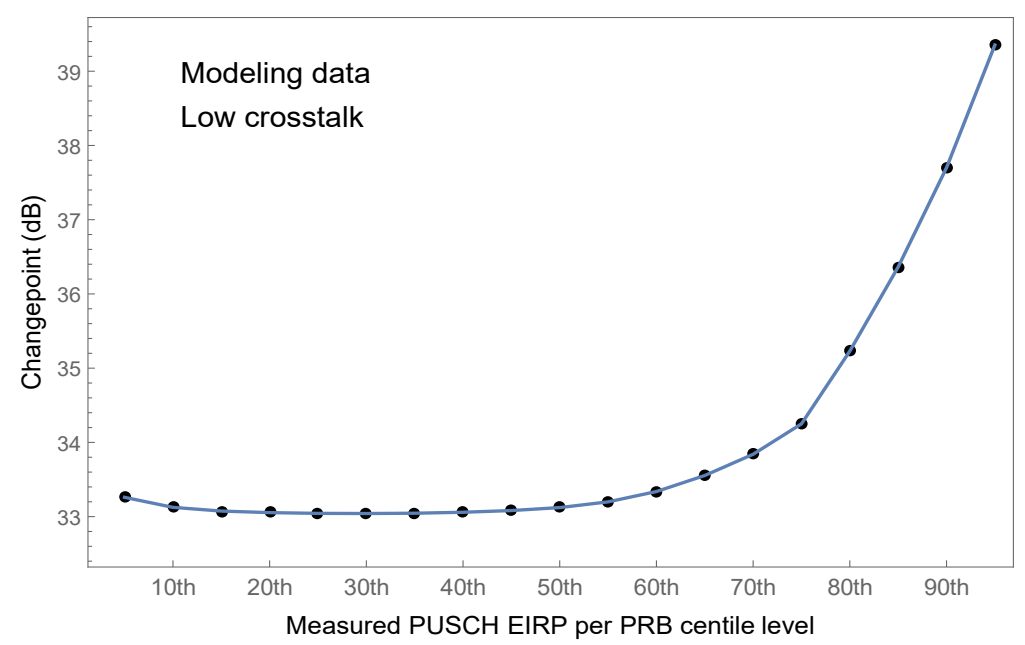

Figure 5.9: Changepoints in the continuous, piecewise linear relationship between measured PUSCH EIRP per PRB centile response and applied attenuation. The changepoints are roughly the same (around $33.5 \mathrm{~dB}$ ) for the bottom $70 \%$ of the measured PUSCH EIRP per PRB distribution, but is greater by as much as $6 \mathrm{~dB}$ for the top $30 \%$ of the power distribution. 


\subsection{Analysis of the modeling data}

Statistical analysis of the data from the modeling experiment is presented in this section. The goal of the analysis is to develop a descriptive model for measured PUSCH EIRP per PRB of the form

$$
Y=f(A, E)+\varepsilon,
$$

where $Y$ is a given centile of measured PUSCH EIRP per PRB, $A$ is applied attenuation, $E$ is the nominal power setting for the eNB, and $\varepsilon$ accounts for random departures from $f(A, E)$. Our aim for model (5.3) is to offer a useful, insightful explanation of $Y$ 's relationship to $A$ and $E$. Ideally, for the purpose of description, the function $f$ in (5.3) would have a familiar parametric form with only a few parameters.

Measured PUSCH EIRP per PRB data were collected for 24 combinations of levels of four ancillary factors $B, C, D$, and $F$, as described in Table 2.1. An unidentified effect is seen at High crosstalk in the data collected in the monitoring experiment, which makes modeling at that level problematic. We therefore limit ourselves to an exploratory analysis of those data, presented in Section 5.4.

Guided by the results of the monitoring experiment, only Low crosstalk data, involving twelve combinations of $C, D$, and $F$, are addressed. If these three ancillary factors have discernible, important effects on the form of $f$ in (5.3) or any of its parameters, as many as twelve different functions $f$ in (5.3) could be needed to describe the relationship of any particular centile $Y$ in (5.3) to $A$ and $E$.

The remainder of this section is organized as follows. First, the effects of different combinations of factors $C, D$, and $F$ on model (5.3) are assessed. Some statistically discernible effects are found, but these are deemed practically unimportant, so one model can satisfactorily describe the relationship of $Y$ to the quantitative factors $A$ and $E$. A hyperbolic paraboloid (HP) model of the relationship is presented that is applicable for applied attenuation $A \leq 30 \mathrm{~dB}$. Finally, a continuous two-piece HP model applicable to all levels of applied attenuation studied in the modeling experiment is proposed. This two-piece model is speculative because of the limited numbers and choices of levels of $A$ and $E$ in the modeling experiment.

\subsubsection{Effects of offered load, scheduling, and the power fraction}

The effects of categorical factors $C, D$, and $F$ on the relationship of $Y$ to the quantitative factors $A$ and $E$ are quantitatively assessed with the ANCOVA model

$$
\begin{aligned}
Y= & \gamma_{0}+\gamma_{1} A+\gamma_{2} E+\gamma_{3} A E \\
& +\gamma_{4} C+\gamma_{5} D+\gamma_{6} F+\gamma_{7} C D+\gamma_{8} D F+\gamma_{9} C F+\delta+\kappa .
\end{aligned}
$$

In the model, $Y$ is the centile of measured PUSCH EIRP per PRB being modeled, the terms with $\gamma_{0}, \gamma_{1}, \gamma_{2}, \gamma_{3}$ express unconstrained linear effects of factors $A$ and $E$, and terms with $\gamma_{4}, \gamma_{5}, \gamma_{6}$ and $\gamma_{7}, \gamma_{8}, \gamma_{9}$ express, respectively, the main and interaction effects of $C, D$, and $F$. Because the experiment is based on a split-plot design, the model has two identifiable sources of random variation, $\delta$ for the whole-plot error and $\kappa$ for sub-plot error [13, 14, 15]. The sub-plot error variance is estimated by the ANCOVA residual mean-squared error; the whole-plot error variance is estimated using the variance associated with $R \times W$, where $R$ is a random block (nuisance factor) identifying the block used for a particular measurement and $W$ is an index that identifies measurements within a whole plot. Whole plots are defined by all possible combinations of $D, E$, and $F$, forming 24 whole plots. (For the purpose of identifying whole plots, $E$ is treated as a categorical factor.) Table 5.6 displays the p-values for each of the effects represented in (5.4), with results for the 50th and 90th response centiles of measured PUSCH EIRP per PRB. Tests for effects associated with $E, D, F$, and $D \times F$ are performed using the whole-plot error variance, while the remaining effects are tested using the sub-plot error variance. 


\begin{tabular}{ccc} 
Effect & $\begin{array}{c}\text { P-value for } \\
\text { 50th centile }\end{array}$ & $\begin{array}{c}\text { P-value for } \\
\text { 90th centile }\end{array}$ \\
\hline \hline$E$ & 0.42 & 0.17 \\
$D$ & 0.15 & 0.83 \\
$F$ & 0.26 & 0.07 \\
$D \times F$ & 0.88 & 0.78 \\
\hline$A$ & 0.00 & 0.00 \\
$A \times E$ & 0.00 & 0.00 \\
$C$ & 0.34 & 0.00 \\
$C \times D$ & 0.02 & 0.11 \\
$C \times F$ & 0.15 & 0.46 \\
\hline \hline
\end{tabular}

Table 5.6: Results for the effects represented in the ANCOVA model (5.4). Effects with p-values below the 0.05 significance level are considered to be discernibly different than zero.

The results in Table 5.6 show for the 50th centile of PUSCH EIRP per PRB, that only $A$ and the interactions $A \times E$ and $C \times D$ are discernible at the 0.05 significance level, and for the 90th centile only $A$ and $C$, singly and in combination, have statistically discernible effects. Also, factor $F$, the eNB power fraction, has no discernible effect, main or interaction, for either the 50th or the 90th centile. These results suggest that, for the 50th centile of measured PUSCH EIRP per PRB, six descriptive models might be needed, one for each $C \times D$ combination, and that for the 90th centile three descriptive models might be needed, one for each level of factor $C$. For fixed levels of $C$ and $D$, with the modeling data restricted to just applied attenuations $A<30 \mathrm{~dB}$, the model in (5.4) is a non-planar quadric surface, the HP

$$
Y=\beta_{0}+\beta_{1} A+\beta_{2} E+\beta_{3} A E+\epsilon .
$$

To assess the import of the discernible effects of $C$ and $D$ identified in Table 5.6, the parameters $\beta_{0}, \beta_{1}, \beta_{2}, \beta_{3}$ in (5.5) are estimated. The estimation is done six times for the 50th centile of measured PUSCH EIRP per PRB, once each for the six subsets of modeling data corresponding to the six combinations of levels of $C$ and $D$ (using just that part of the data with Low crosstalk and $A<30 \mathrm{~dB}$ ). The parameters of a common 50th centile model based on the data from all six combinations of $C$ and $D$ together are also estimated. The parameters in (5.5) are similarly estimated to obtain a common model and three subset models for the 90th centile. The purpose of the analysis is to compare the common models with their corresponding subset models. Specifically, the differences between each common model response surface and its corresponding subset model response surfaces are calculated over the domain of levels of $A$ and $E$. These results for the 50th and 90th centile models are presented in Table 5.7.

The results in Table 5.7 show for the 50th centile that the response surfaces of the six HP subset models deviate from that of the HP common model at most by $0.54 \mathrm{~dB}$ or less and on average by $0.25 \mathrm{~dB}$ or less. Thus, while $C$ and $D$ have discernible effects on the 50th centile HP model, their effects are not large enough to warrant the entailing complication. Similarly, the deviations in Table 5.7 of the response surfaces of the 90th centile subset models for the three levels of $C$ from that of the common model are too small to justify three separate models for the relationship between the 90th centile response and $A$ and $E$. From the standpoint, too, of $R^{2}$ and the RMS errors of the common models versus those of their corresponding subset models, using subset models to describe the relationship of centile response to $A$ and $E$ offers no appreciable advantage.

Based on the comparisons in Table 5.7, the common HP model (5.5) is proposed to describe the relationship of centiles of measured PUSCH EIRP per PRB to the quantitative factors $A$ and $E$. These models are fully detailed in Table 5.8 for the 50th and 90th centiles of measured PUSCH EIRP per PRB, with parameter estimates based on the low crosstalk 


\begin{tabular}{|c|c|c|c|c|c|}
\hline & $\begin{array}{c}\text { Descriptive } \\
\text { HP model }\end{array}$ & $R^{2}$ & $\begin{array}{c}\text { RMS } \\
\text { error }(\mathrm{dB})\end{array}$ & $\begin{array}{c}\text { Average } \\
\text { absolute } \\
\text { deviation }(\mathrm{dB})\end{array}$ & $\begin{array}{c}\text { Maximum } \\
\text { absolute } \\
\text { deviation }(\mathrm{dB})\end{array}$ \\
\hline \multirow[t]{7}{*}{ 50th centile } & Common & $98.7 \%$ & 0.82 & & \\
\hline & $C=10 \%, D=\mathrm{CA}$ & $98.4 \%$ & 0.93 & 0.09 & 0.29 \\
\hline & $C=20 \%, D=\mathrm{CA}$ & $98.6 \%$ & 0.85 & 0.08 & 0.28 \\
\hline & $C=40 \%, D=\mathrm{CA}$ & $98.3 \%$ & 0.94 & 0.12 & 0.32 \\
\hline & $C=10 \%, D=\mathrm{IA}$ & $99.0 \%$ & 0.74 & 0.08 & 0.26 \\
\hline & $C=20 \%, D=\mathrm{IA}$ & $99.1 \%$ & 0.69 & 0.15 & 0.32 \\
\hline & $C=40 \%, D=\mathrm{IA}$ & $99.1 \%$ & 0.65 & 0.25 & 0.54 \\
\hline \multirow[t]{4}{*}{ 90th centile } & Common & $98.7 \%$ & 0.92 & & \\
\hline & $C=10 \%$ & $98.7 \%$ & 0.94 & 0.17 & 0.37 \\
\hline & $C=20 \%$ & $98.9 \%$ & 0.85 & 0.09 & 0.24 \\
\hline & $C=40 \%$ & $98.7 \%$ & 0.93 & 0.17 & 0.23 \\
\hline
\end{tabular}

Table 5.7: Comparison of common and subset descriptive HP models for the 50th and 90th centile measured PUSCH EIRP per PRB responses.

50th centile

\begin{tabular}{|c|c|c|}
\hline \multicolumn{3}{|c|}{$R^{2}=98.7 \%$, RMS error $=0.82 \mathrm{~dB}$} \\
\hline Parameter & Estimate $(\mathrm{dB})$ & $\begin{array}{l}\text { Standard } \\
\text { error }(\mathrm{dB})\end{array}$ \\
\hline$\beta_{0}$ & -31.65 & 0.78 \\
\hline$\beta_{1}$ & 1.136 & 0.047 \\
\hline$\beta_{2}$ & -0.0080 & 0.0080 \\
\hline$\beta_{3}$ & 0.00210 & 0.00060 \\
\hline
\end{tabular}

90th centile

\begin{tabular}{|c|c|c|}
\hline \multicolumn{3}{|c|}{$R^{2}=98.7 \%$, RMS error $=0.92 \mathrm{~dB}$} \\
\hline Parameter & Estimate $(\mathrm{dB})$ & $\begin{array}{c}\text { Standard } \\
\text { error }(\mathrm{dB})\end{array}$ \\
\hline$\beta_{0}$ & -29.80 & 0.78 \\
\hline$\beta_{1}$ & 1.158 & 0.040 \\
\hline$\beta_{2}$ & -0.013 & 0.008 \\
\hline$\beta_{3}$ & 0.00200 & 0.00031 \\
\hline
\end{tabular}

Table 5.8: Fitted HP models for the 50th and 90th centile measured PUSCH EIRP per PRB responses plotted in Fig. 5.10.

modeling data in the range $A \leq 30 \mathrm{~dB}$. The response surfaces of the common HP models for the 50th and 90th centiles of measured PUSCH EIRP per PRB are displayed in Fig. 5.10 with all the low crosstalk data. The plots in Fig. 5.10 show these HP models to be in excellent agreement with the data collected at $A \leq 30 \mathrm{~dB}$. Beyond their empirical good fit, these HP models offer three perspectives on the data. As already noted, the model response surface $f(A, E)$ is geometrically a HP. From a statistical perspective, model (5.5) expresses that $Y$ depends systematically on only firstorder main and interaction effects of $A$ and $E$. And, from a functional perspective, model (5.5) represents unrestricted linear relationships between $Y$ and each of $A$ and $E$. To see this, let $f_{E}(A)=f(A, E)$ be the marginal response $Y$ for fixed $E$ and let $f_{A}(E)=f(A, E)$ be the marginal response $Y$ for fixed $A$. These marginal responses are linear:

$$
\begin{aligned}
& f_{E}(A)=\left(\beta_{0}+\beta_{2} E\right)+\left(\beta_{1}+\beta_{3} E\right) A, \\
& f_{A}(E)=\left(\beta_{0}+\beta_{1} A\right)+\left(\beta_{2}+\beta_{3} A\right) E,
\end{aligned}
$$



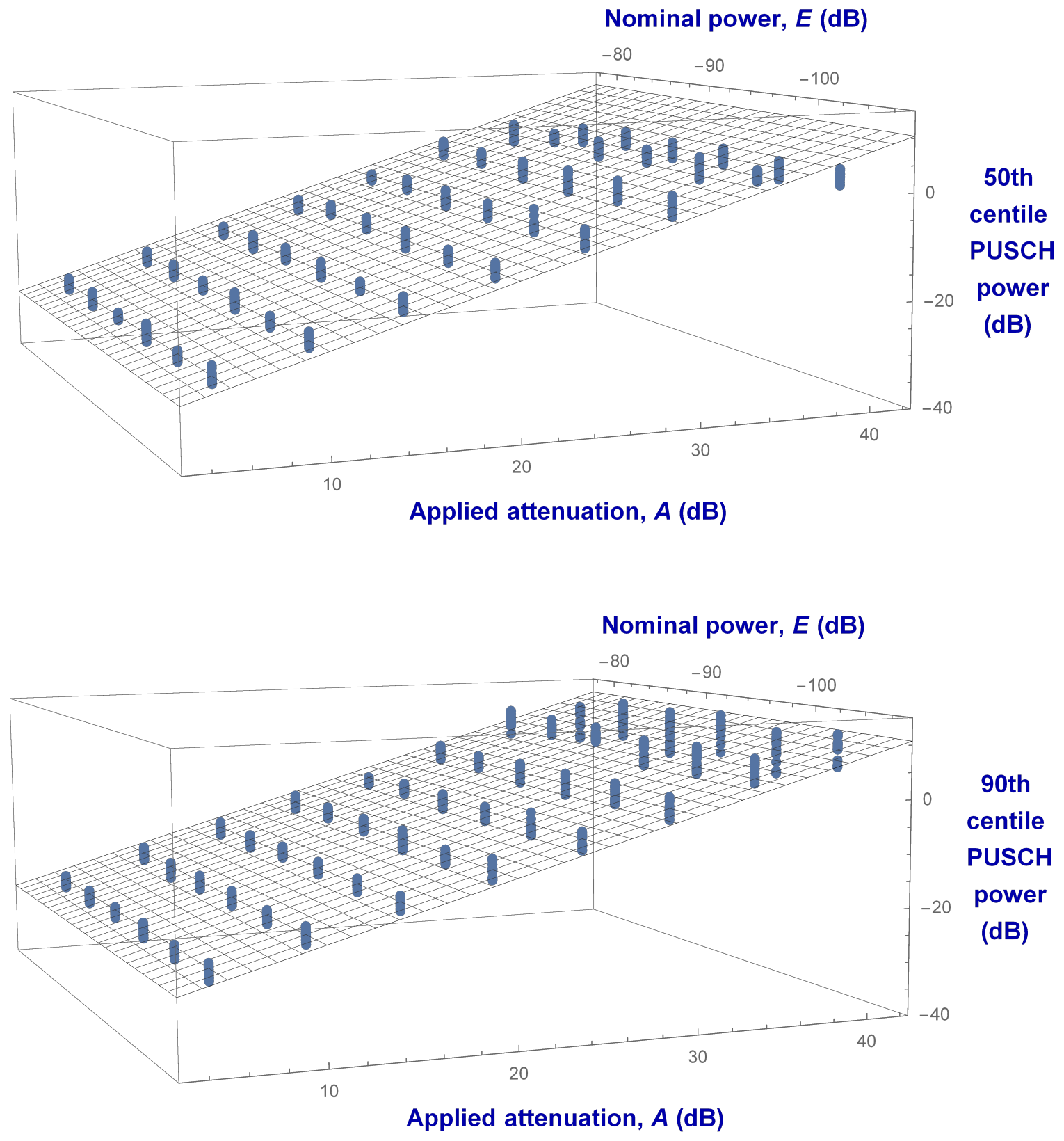

Figure 5.10: Fitted HP models of 50th and 90th centiles (top and bottom, resp.) of measured PUSCH EIRP per PRB, shown with the Low crosstalk modeling data on which these models are based. 
with unconstrained intercepts and slopes; that is, they can vary (linearly) depending on the fixed variable. Finally, $\beta_{3}$ is a measure of how nonplanar the HP response is; $f(A, E)$ is planar with constant-slope marginal responses if and only if $\beta_{3}=0$.

The plots in Fig. 5.10 show that the modeling data with applied attenuation at and above $35 \mathrm{~dB}$ depart from the fitted model in a way that recalls the changepoints present in the modeling of the monitoring data in Fig. 5.8. Not enough data were collected over a fine enough grid around $A=35 \mathrm{~dB}$ to confirm that the analogous feature is present under the different conditions of the modeling experiment. One can speculate, though, that a sharp changeline ${ }^{5}$ does exist in the centile response surface $f(A, E)$, that the response surface is continuous at this changeline, and that for applied attenuations beyond this changeline, the response surface is again a HP. These assumptions are sufficient to imply the centile response model $Y=f(A, E)+\varepsilon$ where $f(A, E)$ is the continuous two-piece HP response surface

$$
f(A, E)=b(A, E)-g(A, E) 1(A-c(E)),
$$

where

$$
\begin{aligned}
& b(A, E)=\beta_{0}+\beta_{1} A+\beta_{2} E+\beta_{3} A E, \\
& g(A, E)=\gamma_{0}+\gamma_{1} A+\gamma_{2} E+\gamma_{3} A E,
\end{aligned}
$$

and $A=c(E)$ is the equation of the changeline, with

$$
c(E)=-\frac{\gamma_{0}+\gamma_{2} E}{\gamma_{1}+\gamma_{3} E} .
$$

The two-piece HP model $f(A, E)$ in (5.6) admits other, equivalent parameterizations. The present parameterization renders the model linear in half of its parameters, making numerically fitting the model less sensitive to starting values and, therefore, more likely to converge to the true minimum RMS error. The first model term $b(A, E)$ represents the HP surface corresponding to low applied attenuation where the surface is increasing. The function $g(A, E)$ is the change in the HP surface that occurs where $A$ crosses the changeline $A=c(E)$.

The continuous, two-piece HP model (5.6) is fitted to the modeling experiment's Low crosstalk data over the full range of applied attenuation settings with the results in Table 5.9 for the 50th and 90th centiles of measured PUSCH EIRP per PRB. The sparsity of the data made these fits difficult to accomplish, and attempts to directly fit model (5.6) to the modeling experiment's 90th centile data failed through lack of convergence and sensitivity to starting values. A combination of homotopy ${ }^{6}$ and the Levenberg-Marquardt procedure ${ }^{7}$ enabled us to achieve the model fits presented here. These fitted centile models are presented in Fig. 5.11 with their corresponding modeling data. Visually, these models fit the data well. The residual plots and and summary quantitative measures in Fig. 5.12 support this interpretation. Other models might fit the data equally well; further data are needed to confirm the existence and nature of the changeline posited by this model.

Figure 5.13 shows the changelines of the 50th and 90th centile two-piece HP models. The changelines in Fig. 5.13 are overlaid on the combinations of levels of $A$ and $E$ from which the changelines are determined. This overlay shows the sparsity of the modeling data collected in the vicinity of the hypothesized changelines, underscoring the speculative nature of the existence and location of these changelines.

\footnotetext{
${ }^{5} \mathrm{~A}$ sharp changeline is a line separating two regions of a surface such that the surface directional derivatives on the line and normal to it are discontinuous.

${ }^{6} \mathrm{~A}$ homotopic solution to a difficult problem is obtained by solving a simple problem and then slowly and smoothly deforming that problem in the direction of the desired problem, at each step re-solving the problem using a perturbation of the solution from the previous step. In the present situation the model fit for the 50th centile data is relatively easy. We used that model fit as a starting point for the model fit for the 51st centile data. Then that model fit is used to start the fit of the 52nd centile data, and so on, up through fitting the 90th centile data.

${ }^{7}$ The Levenberg-Marquardt procedure, sometimes called damped least-squares, is an iterative nonlinear least-squares modeling-fitting algorithm that blends characteristics of gradient descent and the Gauss-Newton algorithm [16, 17].
} 

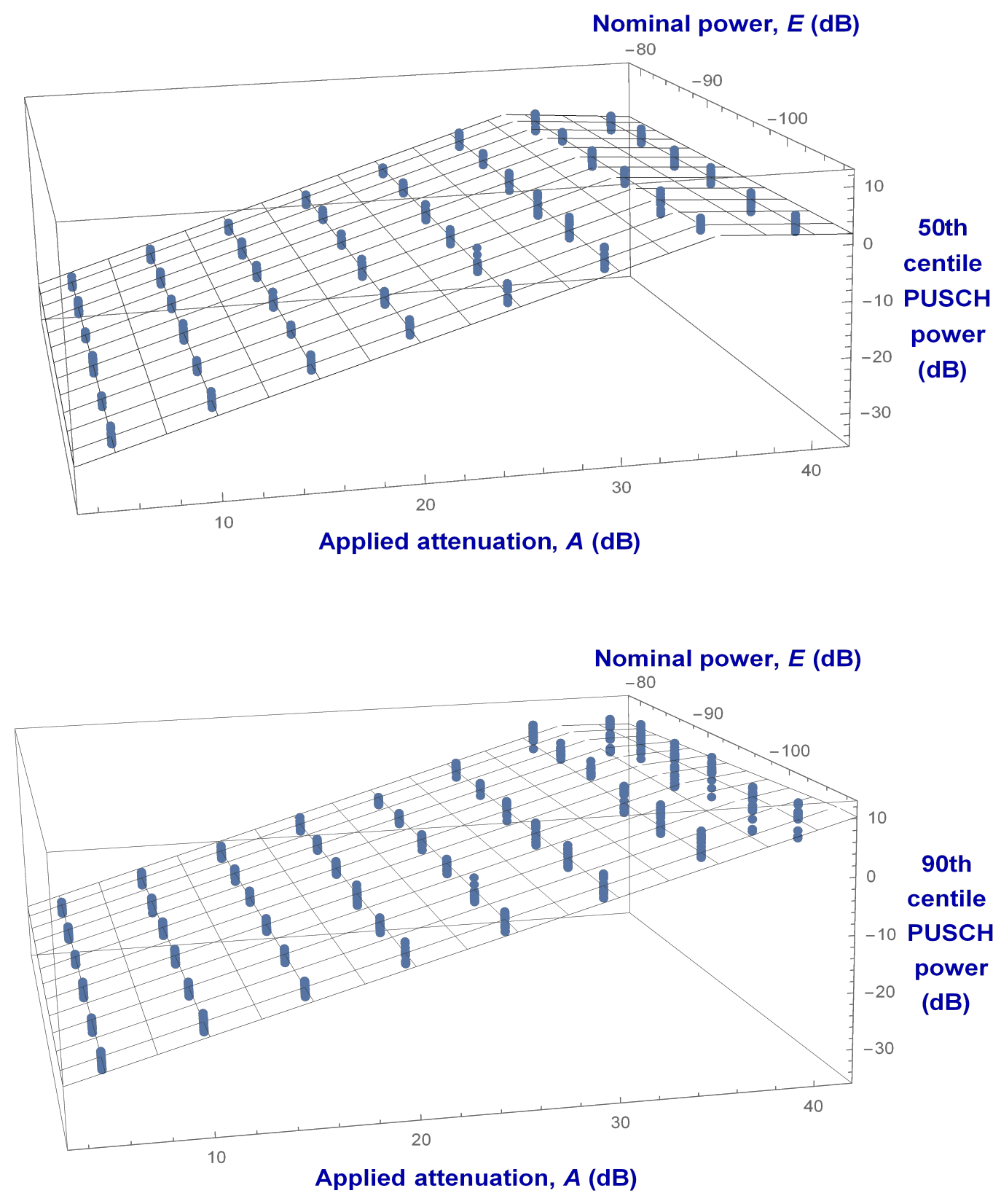

Figure 5.11: Fitted two-piece HP models of 50th and 90th centiles of measured PUSCH EIRP per PRB with the Low crosstalk modeling data on which these models are based. 
50th centile

\begin{tabular}{ccc}
\hline Parameter & Estimate $(\mathrm{dB})$ & $\begin{array}{c}\text { Standard } \\
\text { error }(\mathrm{dB})\end{array}$ \\
\hline$\beta_{0}$ & -33.03 & 0.79 \\
$\beta_{1}$ & 1.254 & 0.040 \\
$\beta_{2}$ & -0.0210 & 0.0085 \\
$\beta_{3}$ & 0.00267 & 0.00043 \\
$\gamma_{0}$ & -49.2 & 9.0 \\
$\gamma_{1}$ & 1.68 & 0.24 \\
$\gamma_{2}$ & -0.082 & 0.097 \\
$\gamma_{3}$ & 0.0049 & 0.0026 \\
\hline
\end{tabular}

90th centile

\begin{tabular}{ccc}
\hline Parameter & Estimate $(\mathrm{dB})$ & $\begin{array}{c}\text { Standard } \\
\text { error }(\mathrm{dB})\end{array}$ \\
\hline$\beta_{0}$ & -30.81 & 0.50 \\
$\beta_{1}$ & 1.234 & 0.055 \\
$\beta_{2}$ & -0.0189 & 0.0076 \\
$\beta_{3}$ & 0.00275 & 0.00060 \\
$\gamma_{0}$ & -99.26 & 0.50 \\
$\gamma_{1}$ & 2.907 & 0.045 \\
$\gamma_{2}$ & -0.8713 & 0.0054 \\
$\gamma_{3}$ & 0.02580 & 0.00049 \\
\hline
\end{tabular}

Table 5.9: Fitted two-piece HP models for the 50th and 90th centile measured PUSCH EIRP per PRB responses plotted in Fig. 5.11.
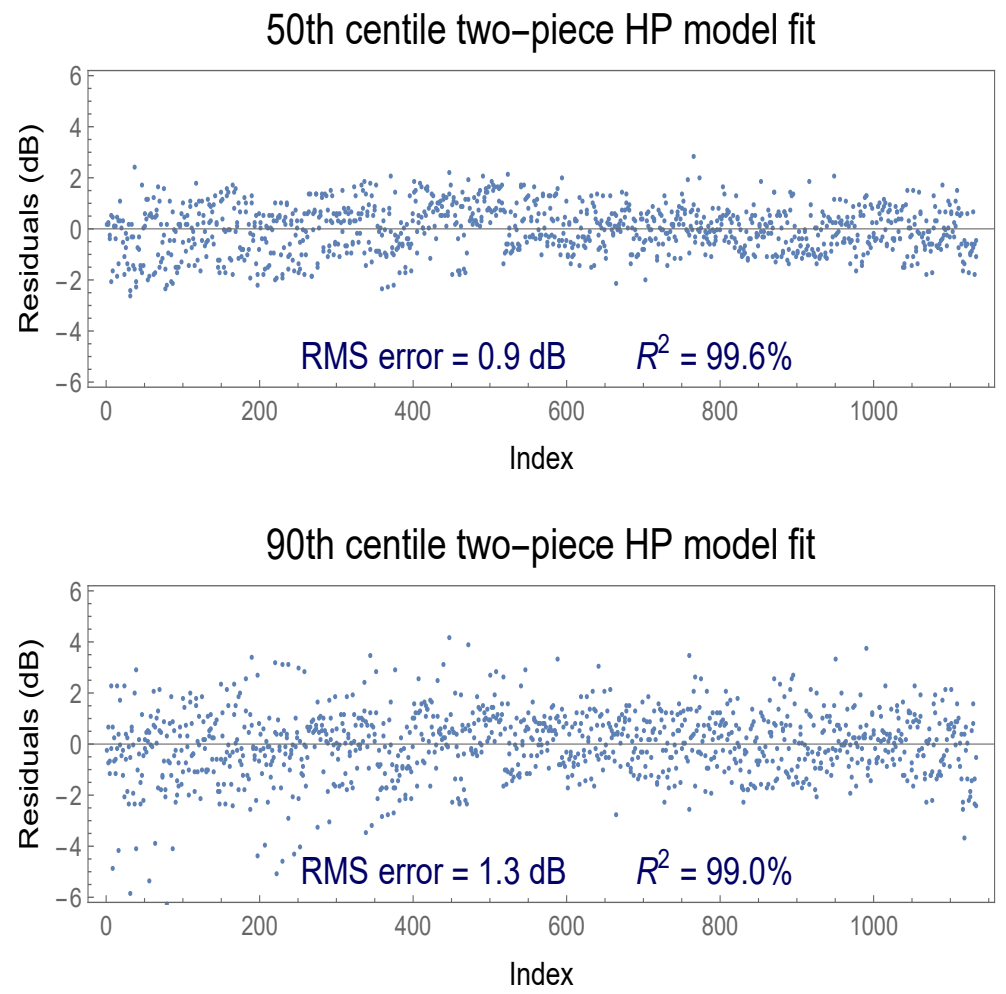

Figure 5.12: Residual plots, RMS errors, and coefficients of determination for the two-piece HP models in Fig. 5.11 and Table 5.9 for the 50th and 90th centile levels (top and bottom, resp.) of measured PUSCH EIRP per PRB. 
The fitted changelines for the 50th and 90th centiles of measured PUSCH EIRP per PRB are shown in Fig. 5.13 along with fitted changelines for the 5th, 75th, and 95th centiles. The five changelines mark applied attenuations corresponding to maximum levels of expected power, and they shift only very slowly rightward (toward higher applied attenuation and higher maximum expected power) up to the 75th centile level. At about the 75th centile level, the changeline begins to shift rightward more rapidly. This recapitulates the rightward shift of the changepoint models captured in Fig. 5.9 for the monitoring data collected at $E=-85 \mathrm{~dB}$. Figure 5.13 suggests that this fast rightward shift may be most pronounced at lower levels of eNB nominal power $E$.

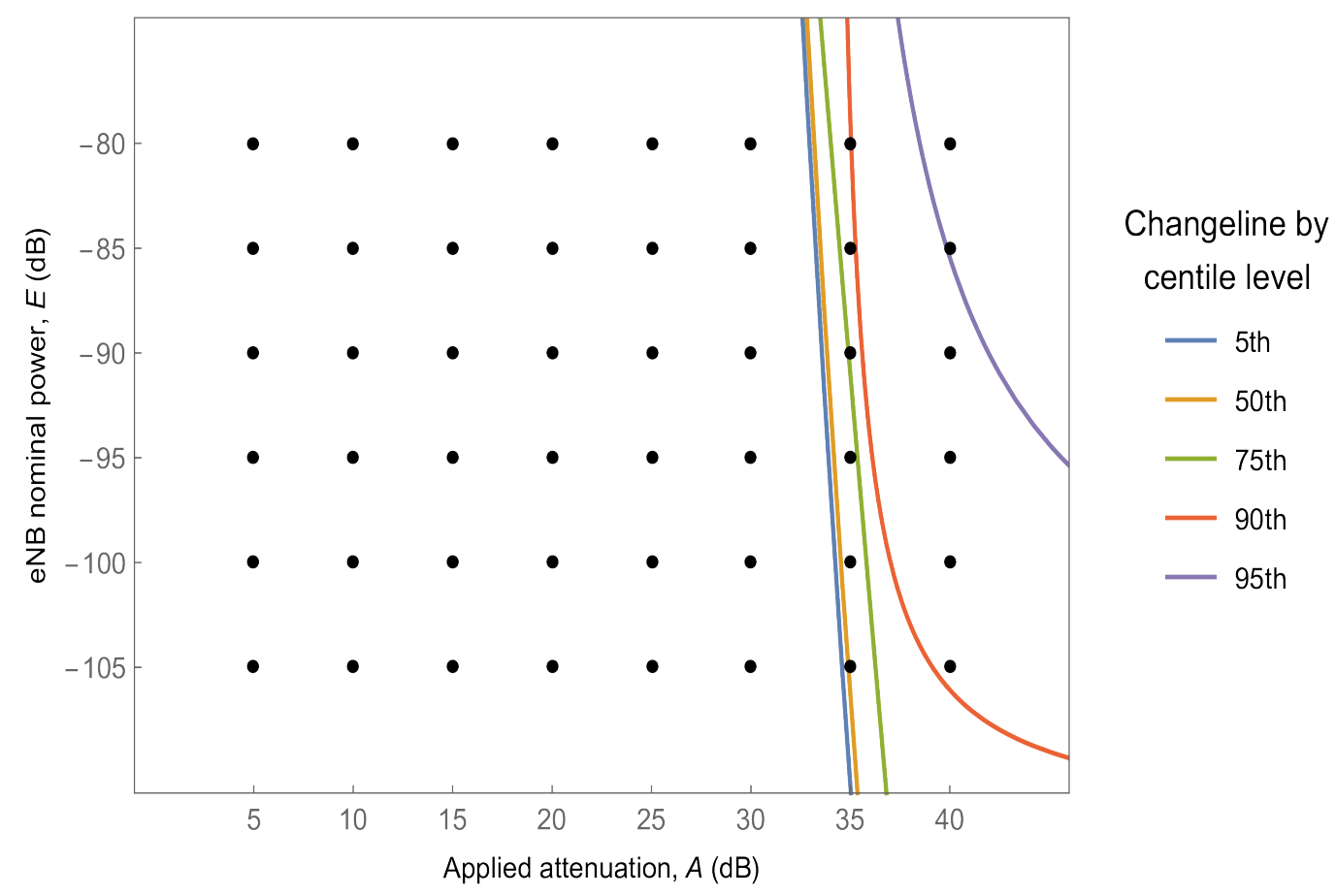

Figure 5.13: Changelines for the fitted two-piece HP model (5.6) at five centile levels, the 50th and 90th centile levels of the models in Fig. 5.11 and, also, the 5th, 75th, and 95th centile levels. The changelines are overlaid on the combinations of $(A, E)$ design points in the CLPC modeling experiment. 


\subsection{High crosstalk in the modeling experiment}

This section presents an exploratory analysis of the data collected with High crosstalk in the CLPC modeling experiment. The key finding of this analysis is that the data collected at High crosstalk show unexpectedly high variability. This finding is consistent with the finding of elevated variability at High crosstalk in the monitoring experiment. An exploratory analysis of the modeling data finds that this variability cannot be accounted for by any of the factors included in the modeling experiment; an unanticipated, unknown variable is at work.

The exploratory analysis begins with the two plots in Fig. 5.14. These plots show all the measured PUSCH EIRP per PRB data (50th and 90th centiles) collected in the modeling experiment at Low crosstalk (left-hand plot) and at High crosstalk (right-hand plot). The plots group measurements by level of applied attenuation $A$. At each level of $A$ in the plots, for each of the two centiles, 144 points are plotted, corresponding to the different replicate combinations of factors $C, D, E$, and $F$. We focus on levels of $A$ below $30 \mathrm{~dB}$, where the relationship of measured PUSCH EIRP per PRB to $A$ is linear. At $A<30 \mathrm{~dB}$ the variability in each of the two centiles of measured PUSCH EIRP per PRB at High crosstalk is 2.5 times the variability at Low crosstalk. This is true both for the 50th and the 90th centiles of measured PUSCH EIRP per PRB.

This additional variability in measured PUSCH EIRP per PRB at High crosstalk could result from a differential effect of one of the study factors $C, D, E$, or $F$; in other words, one of these factors may be interacting with crosstalk $B$. To assess this for factor $C$, offered load, we turn to Fig. 5.15. The plots show measured PUSCH EIRP per PRB at each level of $A$ for High crosstalk, grouped according to the three levels of factor $C$. Focusing on $A<30 \mathrm{~dB}$, no significant reduction is found in the variability in measured PUSCH EIRP per PRB in these $A \times C$ groups compared to that in measured PUSCH EIRP per PRB grouped just according to $A$ in the right-hand plot in Fig. 5.15. This shows that $C$ is not driving the variability in measured PUSCH EIRP per PRB at High crosstalk. Factors $D$, $E$, or $F$ can similarly be assessed to determine whether they are driving the variability in measured PUSCH EIRP per PRB at High crosstalk. Figures 5.16, 5.17, and 5.18 show that none of $D, E$, or $F$ are interacting with crosstalk to account for the
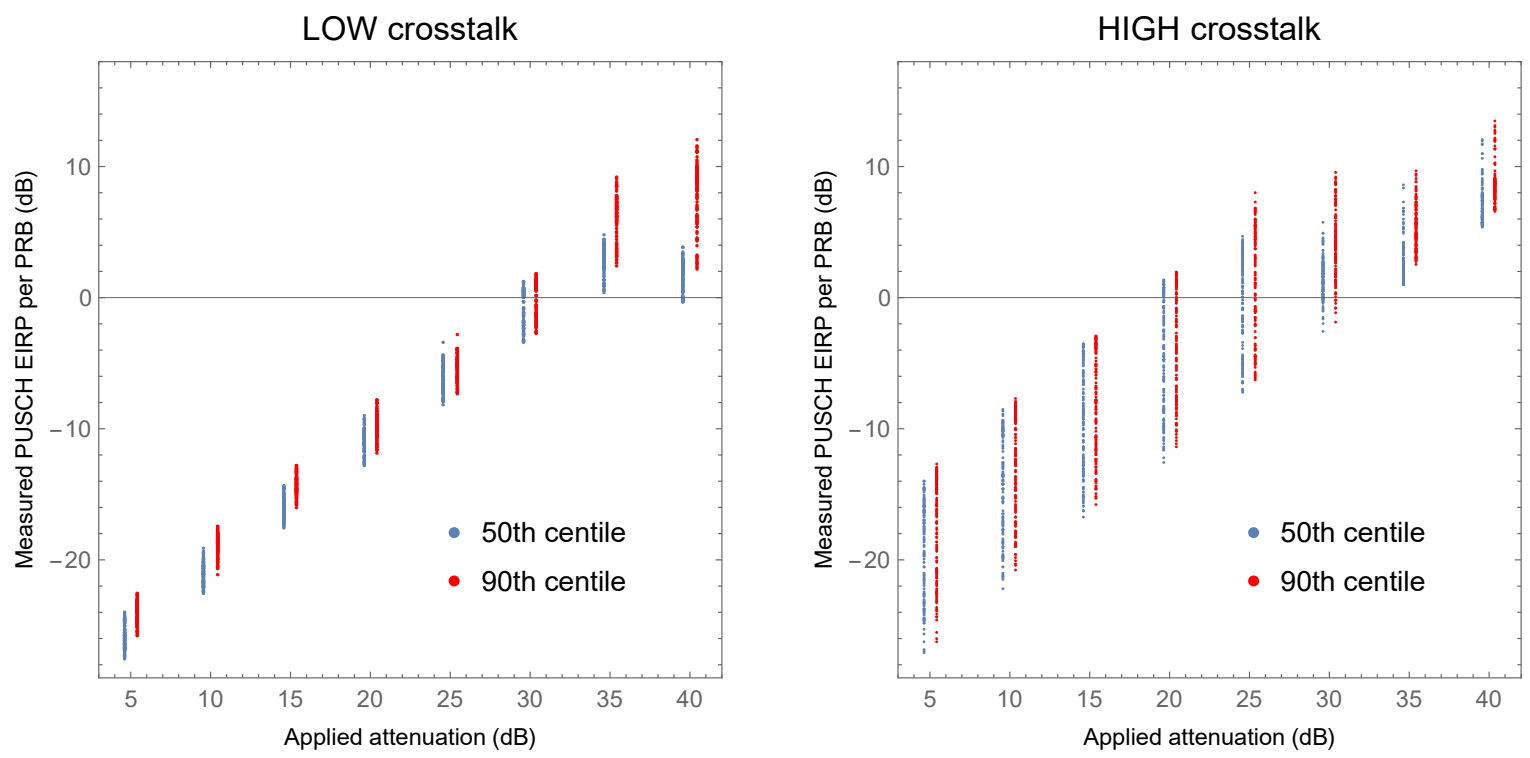

Figure 5.14: Elevated variability in the CLPC modeling experiment in 50th and 90th centiles of measured PUSCH EIRP per PRB at High crosstalk (right) relative to Low crosstalk (left). 
differential variability seen in measured PUSCH EIRP per PRB. An unknown, unanticipated variable not planned for in the modeling experiment is evidently at work, interacting with crosstalk. Further experimentation to 1) confirm this phenomenon, 2) model the relationship of measured PUSCH EIRP per PRB to level of crosstalk, and 3) identify the mechanism/variable that drives this relationship would be interesting.
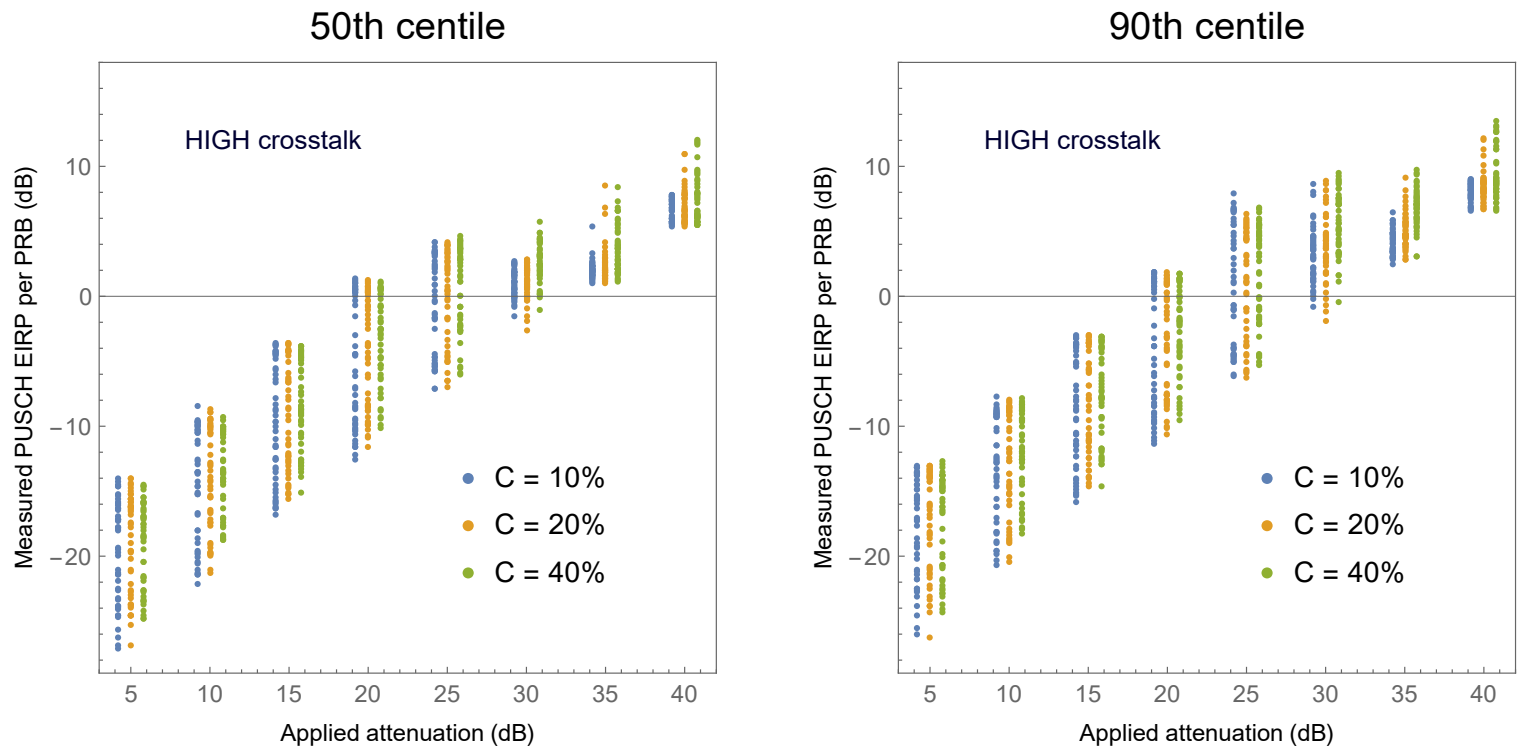

Figure 5.15: 50th and 90th centiles of measured PUSCH EIRP per PRB at High crosstalk in the CLPC modeling experiment. The elevated variability at High crosstalk remains when the modeling data are grouped by fixed levels of network loading factor $C$, indicating that factor $C$ is not the variability's source.
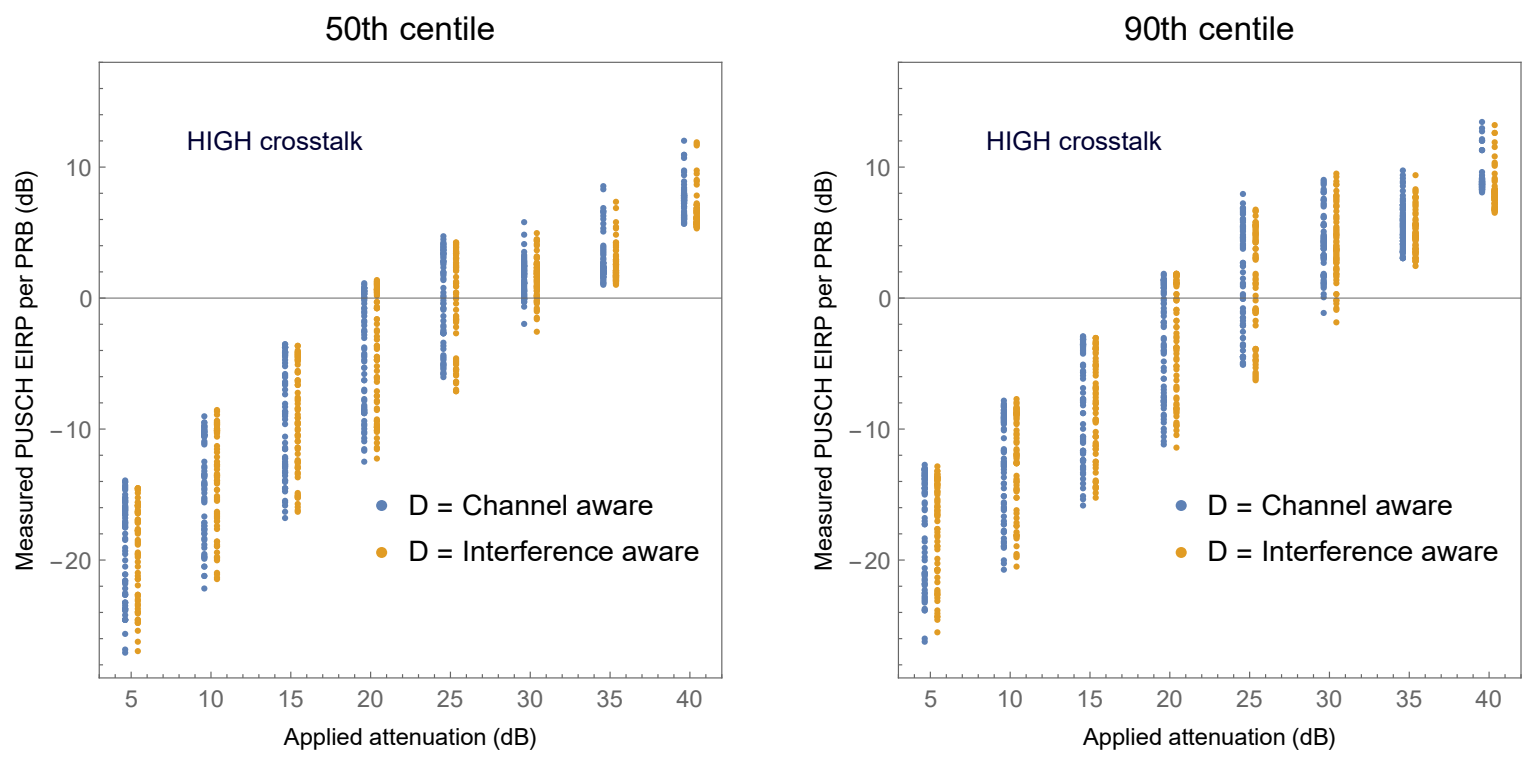

Figure 5.16: 50th and 90th centiles of measured PUSCH EIRP per PRB at High crosstalk in the CLPC modeling experiment. The elevated variability at High crosstalk remains when data are grouped by fixed levels of scheduler type factor $D$, indicating that factor $D$ is not the variability's source. 

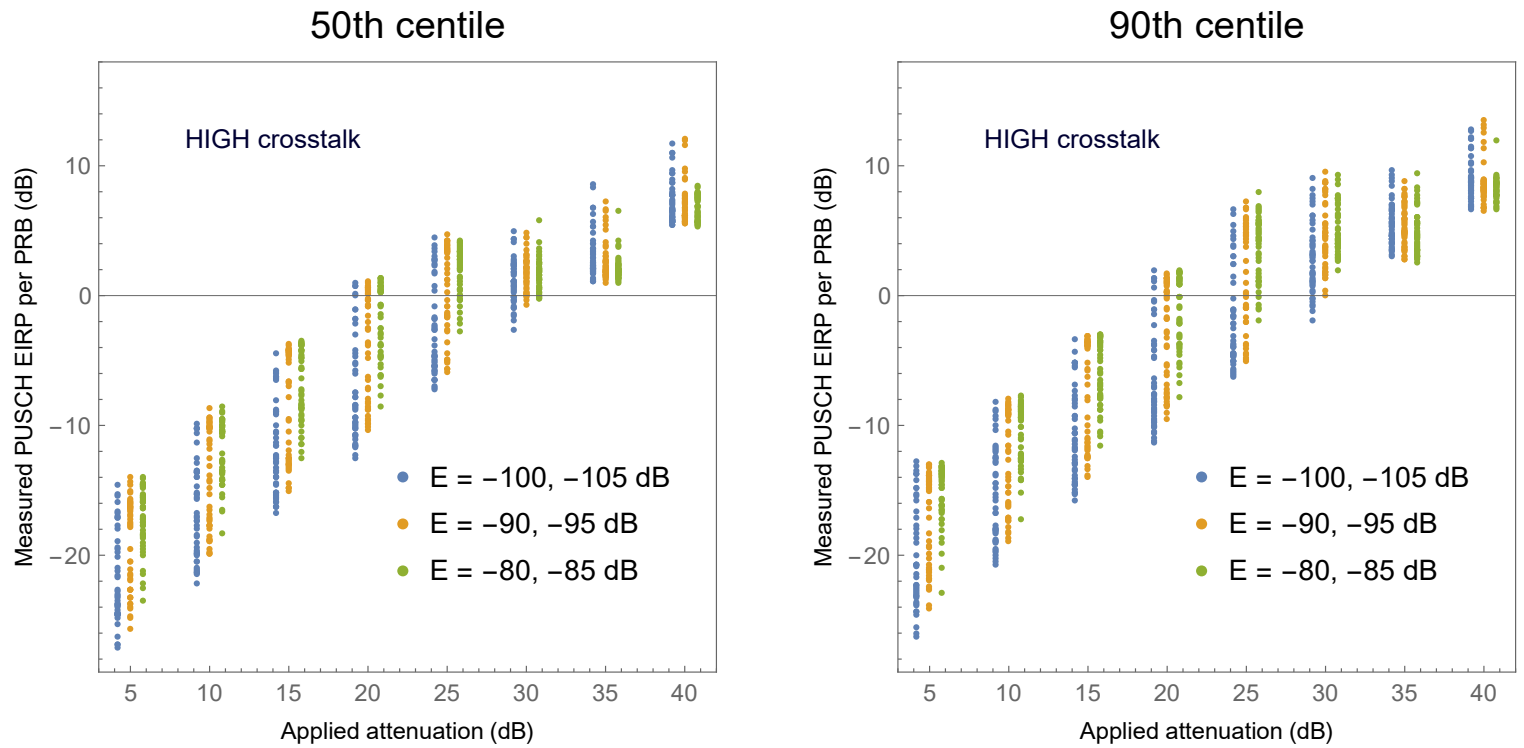

Figure 5.17: 50th and 90th centiles of measured PUSCH EIRP per PRB at High crosstalk in the CLPC modeling experiment. The elevated variability at High crosstalk remains when data are grouped by fixed levels of FPC nominal power factor $E$, indicating that factor $E$ is not the variability's source.
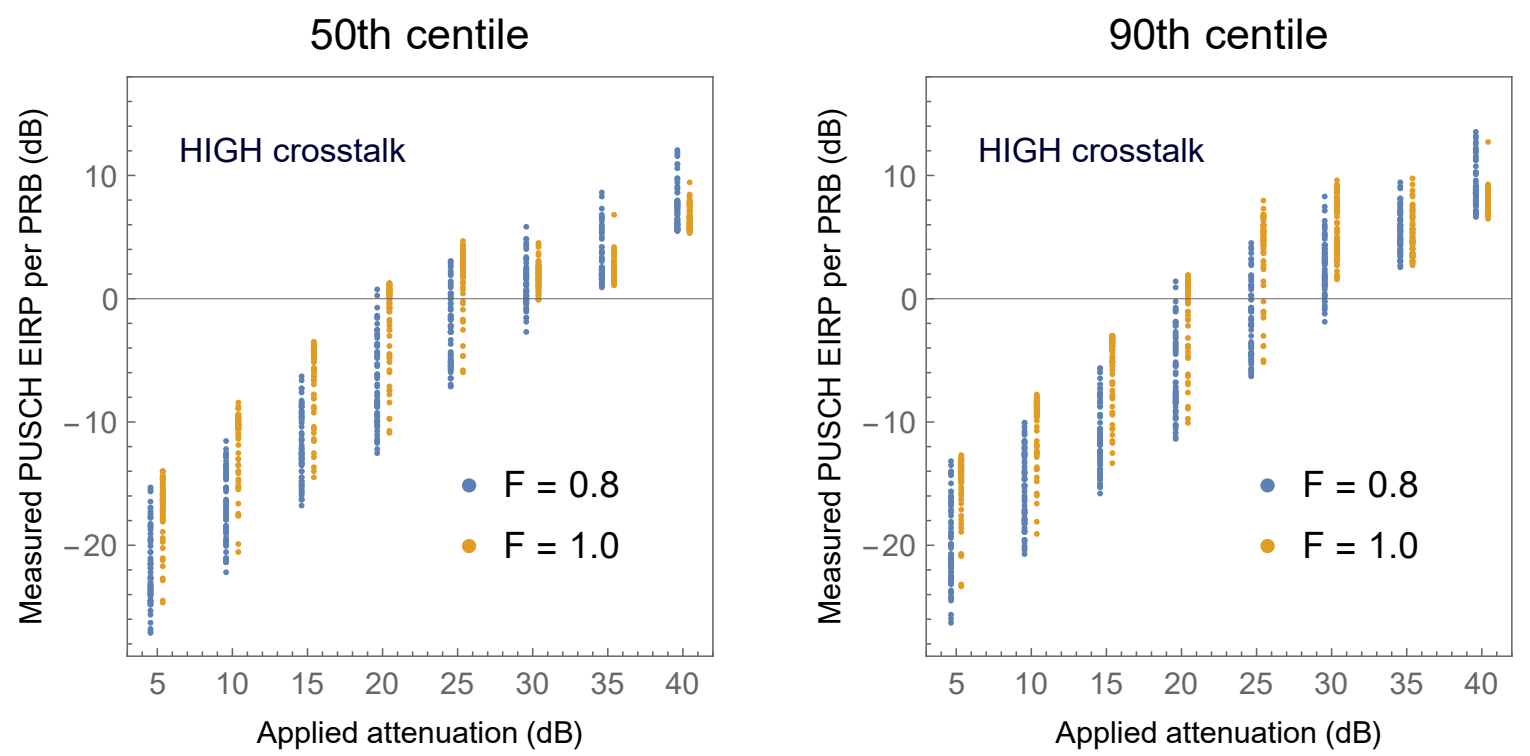

Figure 5.18: 50th and 90th centiles of measured PUSCH EIRP per PRB at High crosstalk in the CLPC modeling experiment. The elevated variability at High crosstalk remains when data are grouped by fixed levels of FPC power fraction factor $F$. 


\subsection{Conclusions of statistical analysis}

The closed-loop power control study involved two experiments: a modeling experiment to create descriptive models of measured PUSCH EIRP per PRB and a monitoring experiment conducted in parallel with the modeling experiment for the primary purpose of checking for instability in the testbed. In summary, the main statistical findings of these two experiments are:

- The monitoring experiment found that, at the fourteen time points where monitoring data were collected over nearly two months of operation, the PUSCH EIRP per PRB measured in the testbed exhibited no discernible long-term trends; in short, the testbed was stable over the set of fourteen time points where it was used.

- PUSCH EIRP per PRB measured in the monitoring experiment exhibited inexplicably large short-term variation at the experiment's High crosstalk setting. The variation seems to originate with an unidentified phenomenon/variable native to LTE communication under this condition. Further experiments are needed to isolate the source and nature of this phenomenon and assess the extent of its effect on measured PUSCH EIRP per PRB.

- Four copies of a single model of UE were used in the two CLPC experiments. This UE was a moderately priced model, and the four copies were purchased from different vendors. The replication designed into the monitoring experiment is sufficient to assess the variation contributed by any physical differences among the four handsets. No statistically discernible handset-contributed variation is found in the measured PUSCH EIRP per PRB.

- In both experiments offered load had either no statistically discernible effect or, where an effect was detected, its size was a fraction of $1 \mathrm{~dB}$. This allowed descriptive models for measured PUSCH EIRP per PRB to be constructed with no need to account for offered load.

- Continuous, piecewise linear models are proposed to describe the centiles of measured PUSCH EIRP per PRB in the monitoring experiment. The models, by standard quantitative measures, excellently describe the centiles of measured PUSCH EIRP per PRB. The key feature of these models is a changepoint in applied attenuation where the power centile reaches a maximum and then declines. Surprisingly, the changepoint varies with centile level, increasing dramatically for centiles in the upper $30 \%$ of the PUSCH EIRP per PRB distribution. The increase in changepoint has the effect of adding as much as $6 \mathrm{~dB}$ to the ceiling on the top $30 \%$ of the PUSCH EIRP per PRB distribution.

- The goal of the modeling experiment is to descriptively model the centiles of measured PUSCH EIRP per PRB in terms of both applied attenuation and eNB nominal power, accounting as necessary for four ancillary factors, crosstalk $B$, offered load $C$, scheduler type $D$, and eNB power fraction $F$. Based on results from the monitoring experiment, only data collected for Low crosstalk are used for modeling, eliminating $B$ as a factor. The three ancillary factors $C, D$, and $F$ were found to have either no statistically discernible effect or, where an effect was detected, its size is a fraction of $1 \mathrm{~dB}$. Therefore, for the data collected in the modeling experiment, no accounting for the effects of these four ancillary factors is included in the proposed centile models of measured PUSCH EIRP per PRB.

- The proposed models of measured PUSCH EIRP per PRB describe its relationship to applied attenuation $A$ and eNB nominal power $E$ as a quadric response surface, taking, specifically, the form of a HP. These models provide, by standard measures, excellent fits to the modeling data collected at levels of applied attenuation at and below $30 \mathrm{~dB}$. At levels of $A$ above $30 \mathrm{~dB}$, the data depart from the model in a way similar to that seen in the more restricted setting of the continuous, piecewise linear models from the monitoring experiment. Guided by this observation, one can speculatively propose continuous, two-piece, HP models of centile response for the full range of collected modeling data. Data with a fuller set of levels of $A$ and $E$ are required to confirm these speculated models. 


\section{Chapter 6}

\section{Engineering Analysis}

\subsection{Introduction}

This chapter is dedicated to the engineering analysis of the closed loop power control experiment. The goal of this chapter is to supplement the findings of the formal statistical analysis in chapter 5, by recasting the formal statistical design of experimental factors into physical properties. In addition, the results of the formal statistical analysis are contextualized using expanded diagnostic responses from the USRD. This chapter is organized as follows:

- Experimental results in the context of the LTE PUSCH power control equation

- Comparison of measured EIRP and USRD-reported power per PRB

- Additional analysis of high crosstalk cases

- Dynamics of negative power headroom

\subsection{Experimental results in context of the power control equation}

In our previous report, "Characterizing LTE User Equipment Emissions: Factor Screening" [1], we found that 14 out of the 28 tested factors had a statistically-significant effect on the median output power of the UE. Furthermore, the seven factors summarized in Table 6.1 were found to have an effect of $3 \mathrm{~dB}$ or larger. These factors heavily influenced the selection of factors for this experiment. Whereas the study in [1] was broad and involved large changes in factor settings to maximize effect sizes, this experiment focused on PUSCH emissions in closed-loop power control mode, using a more realistic ranges of factor settings; see Chapter 4 for details on the experimental design.

As described in [1], PUSCH EIRP per PRB was measured with a VSA and corrected to reflect power emissions at a plane outside of the DUT UE over two $5 \mathrm{~s}$ captures for each experimental configuration. In addition, the USRD provided several other data streams reported each subframe. These can be analyzed simultaneously with the EIRP data to broaden understanding of the UE emissions.

Three of the experimental factors ( $P_{0}, \alpha$, path loss) are parameters in the equation governing LTE PUSCH closed-loop

Table 6.1: Two-level factors and settings from the screening experiment results reported in [1] with the largest effect on median EIRP PUSCH per PRB

\begin{tabular}{cccc}
\hline \hline Factor & Factor Code Used in [1] & Factor Values & Effect on Median $(\mathrm{dB})$ \\
\hline Power Control Type & $\mathrm{K}$ & Open Loop or Closed Loop & $9 \mathrm{~dB}$ \\
PUSCH Power Control, $P_{0}$ & $\mathrm{O}$ & $-85 \mathrm{dBm}$ or $+24 \mathrm{dBm}$ & $9 \mathrm{~dB}$ \\
Fractional Power Control, $\alpha$ & $\mathrm{P}$ & 0.5 or 1.0 & $7 \mathrm{~dB}$ \\
Max Uplink Transmission Power & $\mathrm{S}$ & $0 \mathrm{dBm}$ or $+24 \mathrm{dBm}$ & $8 \mathrm{~dB}$ \\
Outer Loop Link Adaptation & $\mathrm{W}$ & Deactivated or Activated & $3 \mathrm{~dB}$ \\
Cell Scheduling Request Periodicity & $\mathrm{Y}$ & 1 or 100 & $5 \mathrm{~dB}$ \\
Target uplink Outer Scheduling & $\mathrm{b}$ & Lower Edge or None & $-8 \mathrm{~dB}$ \\
\hline
\end{tabular}


power control [7, Sec. 5.1.1],

$$
P_{\mathrm{CL}-\mathrm{PUSCH}}=\min \left[P_{c M a x}, 10 \log _{10}\left(M_{\mathrm{PUSCH}}(i)\right)+P_{0 \mathrm{PUSCH}}(i)+\mathrm{PL} \alpha(i)+\Delta_{T F}+f(i)\right],
$$

where

- $P_{C M a x}$ is the maximum UE transmit power,

- $M_{\mathrm{PUSCH}}$ is the number of PUSCH resource blocks assigned by the scheduler,

- $P_{0 \text { PUSCH }}$ is the nominal power that eNB expects to receive from a UE,

- PL is the downlink path loss between the eNB and UE,

- $\alpha$ is the fractional power control parameter,

- $\Delta_{T F}$ is a modulation coding scheme (MCS)-dependent parameter; for all experiments covered in this report, this factor was disabled, so $\Delta_{T F}=0$,

- $f(i)$ is the transmission power control (TPC) accumulator value.

For additional details on the LTE PUSCH power control specification, see [7, Sec. 5.1.1]. Note that the path loss (PL) in the LTE power control equation is specified as the downlink path loss, which is assumed to be identical to the uplink path loss. In our testbed, a discrepancy was found between the uplink and downlink path loss values measured independently using a VNA, and also between the downlink path loss calculated from RSRP, as specified in the 3GPP standard [7, Sec. 5.1.1], and the downlink path loss reported in the USRD. See Table 6.2 for tabulated values and Figure 6.1 for a graphical representation. Since path loss is the independent variable in the power control equation, the observed differences have implications for predictions based on the power control equation. Specifically, although the UE has a self-consistent self-reported power, the measured power emitted from the UE disagrees with the power control equation evaluated with independent measurements of path loss.

In Chapter 5, it was found that the results could be logically separated by the crosstalk factor, and in cases of low crosstalk, that measured EIRP per PRB behaved linearly below a threshold or change point. This linear behavior is similar to what one would expect in open-loop power control. In the open-loop case, the power control equation simplifies to

$$
P_{\text {OpenLoopPUSCH }}-10 \log _{10}\left(M_{\mathrm{PUSCH}}(i)\right)=P_{0 \text { PUSCH }}(i)+\operatorname{PL} \alpha(i) \text {. }
$$

Above, the power per PRB is linear with a slope equal to the fractional power control factor, $\alpha$ and an intercept at zero path loss equal to $P_{0}$. In Figure 6.2, we plot this relationship for the monitoring experiment, denoting $P_{0}, \alpha$, and the approximate path loss range for the experiment.

We can gain insight by comparing the closed-loop response to the expected open-loop behavior. Namely, using the a path loss offset of $78.1 \mathrm{~dB}$ derived from the VNA downlink measurements (see Table 6.2), we can plot the open loop expectation, the experimental data, and the fit from the analysis in Chapter 5 on the same axis. Figure 6.3 reveals that the empirical behavior of PUSCH EIRP in both the modeling and monitoring experiments is equivalent to $P_{0} \approx-108 \mathrm{dBm}$ and $\alpha \approx 1$. It is important to note this approximate behavior depends on the centile fit, and from Tables 5.6 and 5.8, this can be deduced. Additionally, it is interesting to note that for the closed-loop, low-crosstalk cases shown here, the lack of sensitivity to the $P_{0}$ and $\alpha$ settings indicates that the effects observed in [1] for $P_{0}$ and $\alpha$ are likely underestimated compared to the true effects in the open-loop case, since the analysis in [1] aggregates those effects across open-loop and closed-loop conditions. 
Table 6.2: Table of Relative Path Loss Values with 95 Centile Confidence Intervals

\begin{tabular}{lllll}
\hline \hline Attenuator Setting & $\begin{array}{l}\text { Path Loss Calculated } \\
\text { from RSRP }\end{array}$ & USRD Path loss & $\begin{array}{l}\text { EIRP Downlink Path } \\
\text { Loss }\end{array}$ & $\begin{array}{l}\text { EIRP Uplink Path } \\
\text { Loss }\end{array}$ \\
\hline \hline & & & & \\
$0.0 \pm .5 \mathrm{~dB}$ & $83 \pm 2 \mathrm{~dB}$ & $90 \pm 6 \mathrm{~dB}$ & $78.1 \pm 1.6 \mathrm{~dB}$ & $73.7 \pm 1.0 \mathrm{~dB}$ \\
$5.0 \pm .5 \mathrm{~dB}$ & $88 \pm 3 \mathrm{~dB}$ & $95 \pm 6 \mathrm{~dB}$ & $83.1 \pm 1.6 \mathrm{~dB}$ & $78.7 \pm 1.0 \mathrm{~dB}$ \\
$10.0 \pm .5 \mathrm{~dB}$ & $93 \pm 3 \mathrm{~dB}$ & $100 \pm 7 \mathrm{~dB}$ & $88.1 \pm 1.7 \mathrm{~dB}$ & $83.7 \pm 1.0 \mathrm{~dB}$ \\
$15.0 \pm .5 \mathrm{~dB}$ & $98 \pm 2 \mathrm{~dB}$ & $105 \pm 7 \mathrm{~dB}$ & $93.1 \pm 1.7 \mathrm{~dB}$ & $88.5 \pm 1.0 \mathrm{~dB}$ \\
$20.0 \pm .5 \mathrm{~dB}$ & $102 \pm 3 \mathrm{~dB}$ & $110 \pm 7 \mathrm{~dB}$ & $98.0 \pm 2.2 \mathrm{~dB}$ & $93.2 \pm 1.2 \mathrm{~dB}$ \\
$25.0 \pm .5 \mathrm{~dB}$ & $107 \pm 3 \mathrm{~dB}$ & $115 \pm 7 \mathrm{~dB}$ & $102.7 \pm 2.8 \mathrm{~dB}$ & $98.1 \pm 1.7 \mathrm{~dB}$ \\
$30.0 \pm .5 \mathrm{~dB}$ & $112 \pm 3 \mathrm{~dB}$ & $120 \pm 7 \mathrm{~dB}$ & $107.2 \pm 4.8 \mathrm{~dB}$ & $102.8 \pm 2.6 \mathrm{~dB}$ \\
$35.0 \pm .5 \mathrm{~dB}$ & $117 \pm 3 \mathrm{~dB}$ & $125 \pm 7 \mathrm{~dB}$ & $112.5 \pm 3.0 \mathrm{~dB}$ & $107.9 \pm 1.6 \mathrm{~dB}$ \\
$37.5 \pm .5 \mathrm{~dB}$ & $120 \pm 2 \mathrm{~dB}$ & $127 \pm 7 \mathrm{~dB}$ & -- & -- \\
$40.0 \pm .5 \mathrm{~dB}$ & $123 \pm 2 \mathrm{~dB}$ & $131 \pm 6 \mathrm{~dB}$ & $117.9 \pm 2.6 \mathrm{~dB}$ & $113.1 \pm 2.3 \mathrm{~dB}$ \\
$42.5 \pm .5 \mathrm{~dB}$ & $125 \pm 2 \mathrm{~dB}$ & $132 \pm 6 \mathrm{~dB}$ & -- & -- \\
$45.0 \pm .5 \mathrm{~dB}$ & $128 \pm 2 \mathrm{~dB}$ & $134 \pm 6 \mathrm{~dB}$ & $122 \pm 8 \mathrm{~dB}$ & $118 \pm 4 \mathrm{~dB}$ \\
\hline
\end{tabular}

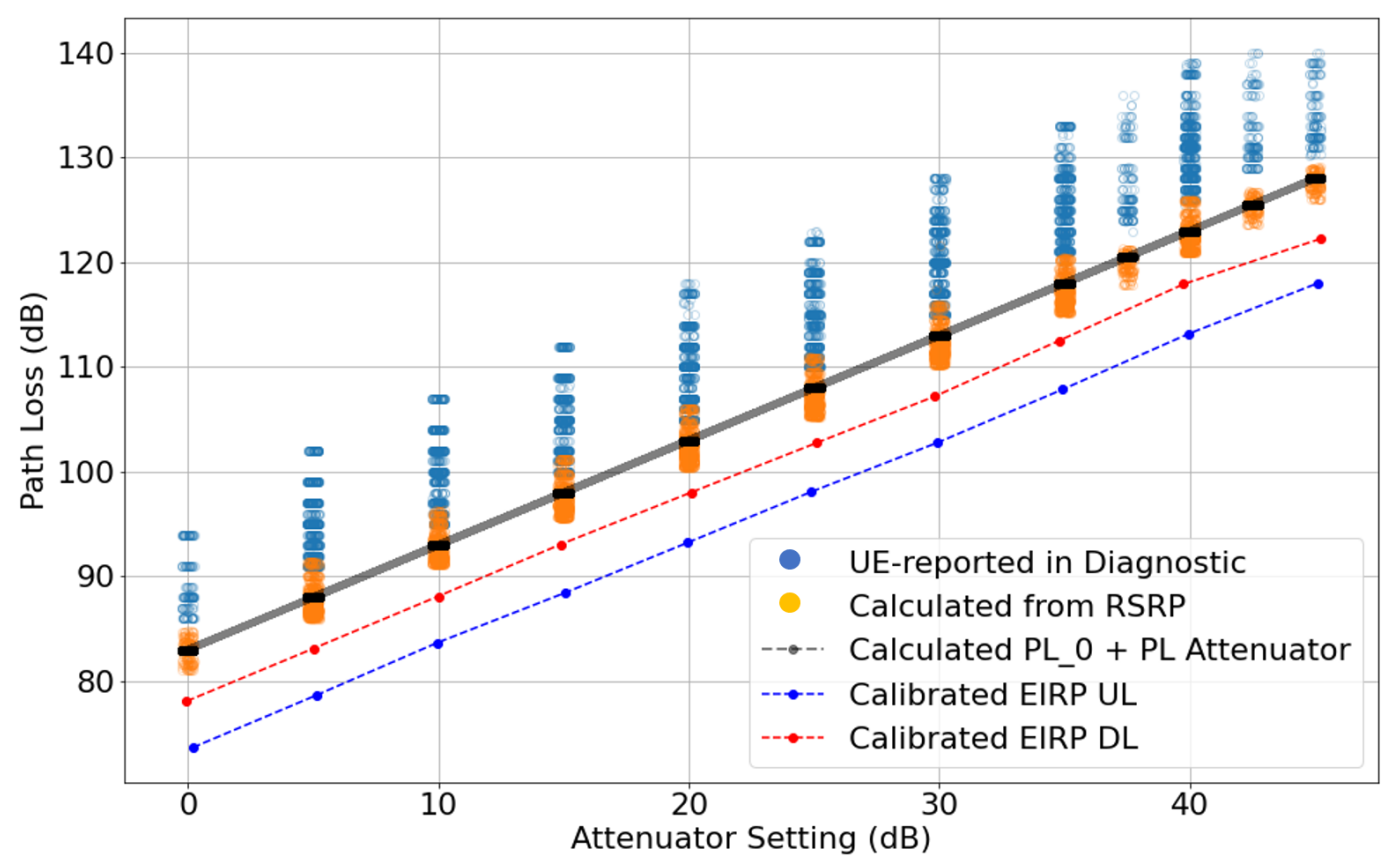

Figure 6.1: Relative Path Loss Calculations versus Factor A: Attenuator Setting 


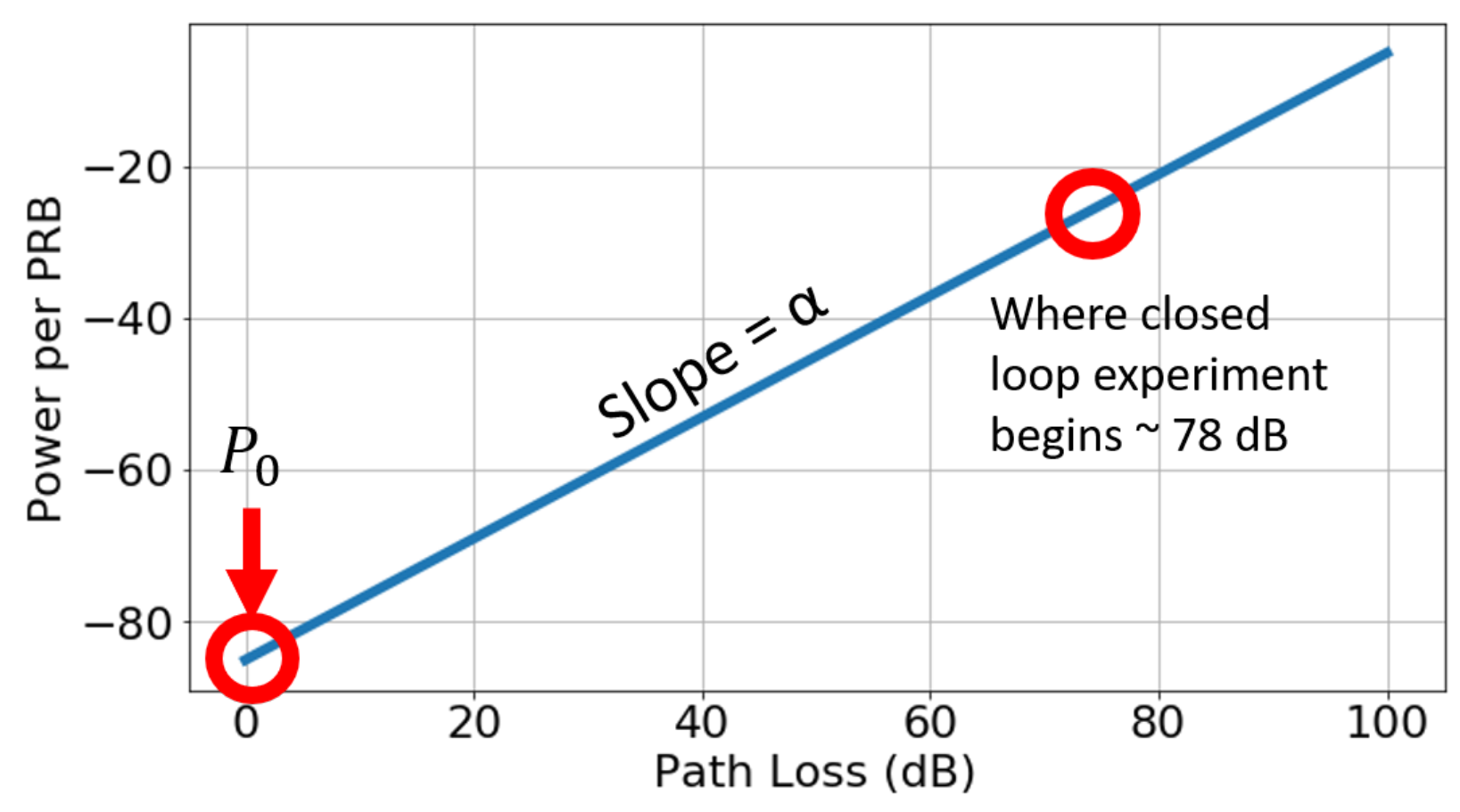

Figure 6.2: Plot of Open Loop Expectation.

To clarify the issues around the power control behavior, a single exploratory data set was collected under the monitoring experimental conditions with the exception that power control was set to open loop ${ }^{1}$. The low crosstalk data from this experiment is shown in Figure 6.4. The measured PUSCH power per PRB exceeds the open loop expectation. The reported data when corrected by $90 \mathrm{~dB}$ for the path loss measured by the UE (see table 6.2) is consistent with the power control equation. We conclude that the large discrepancy in the path loss measured by the UE compared to other methods leads the UE to emit a higher power per PRB than expected by only using equation 6.1.

\footnotetext{
${ }^{1}$ These data were collected from the test circuit and hardware described in this report. These data were not reused from the prior factor screening experiment
} 


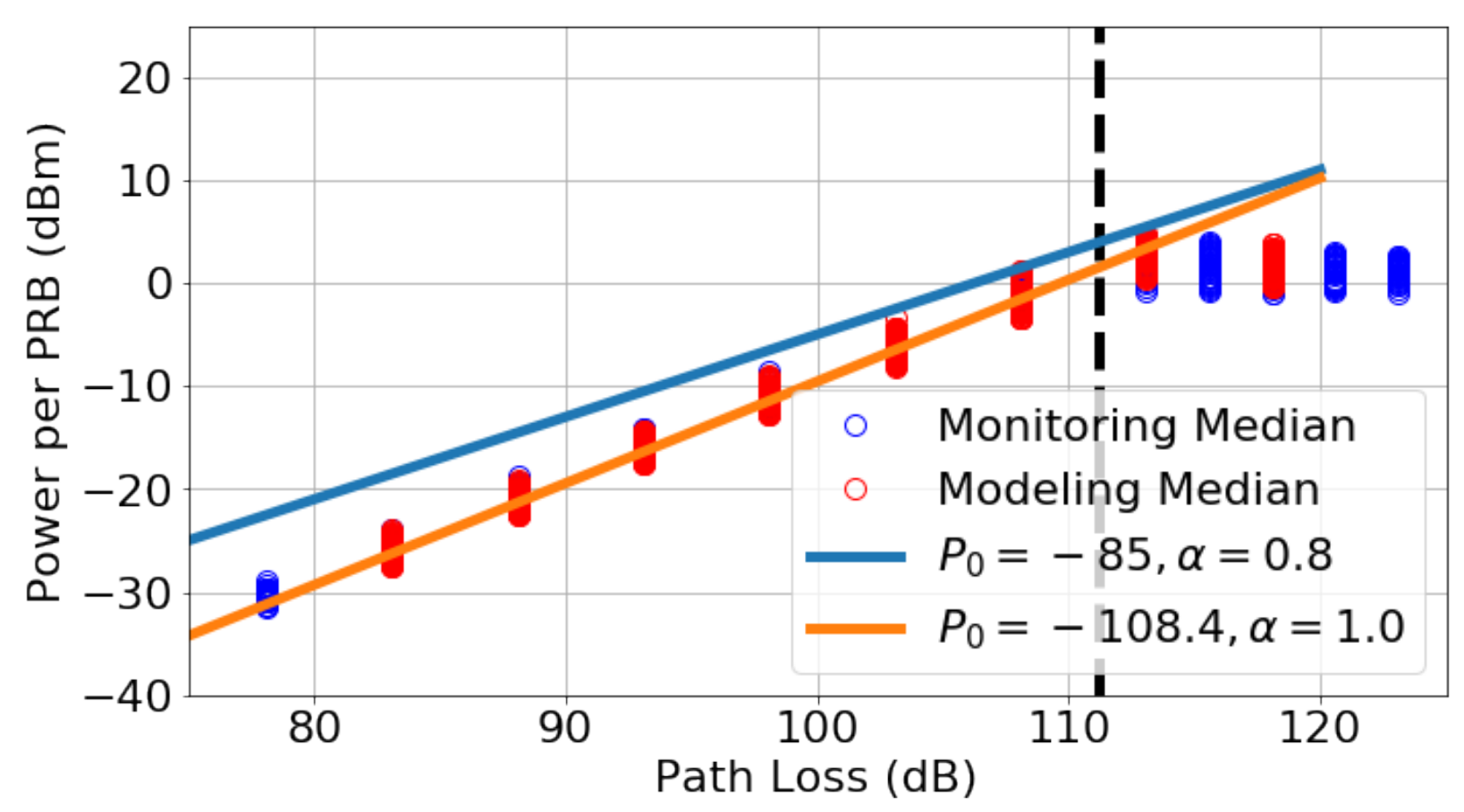

Figure 6.3: Plot of open loop expectation (blue line), fit from chapter 5 (orange line) with change point (vertical dashed line), and experimental data (blue and red circles) for low crosstalk monitoring and modeling experiment.

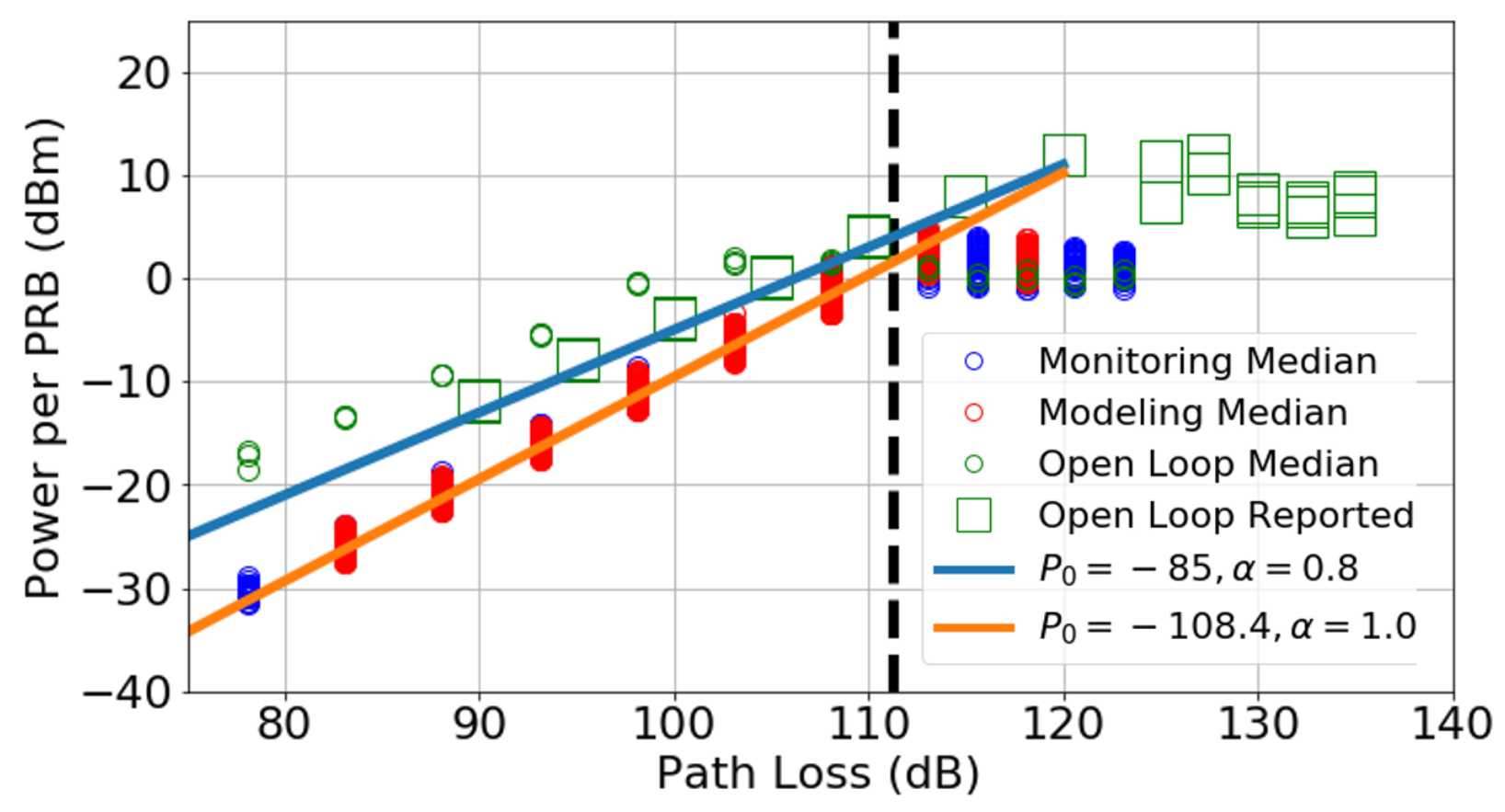

Figure 6.4: Plot of open loop expectation (blue line), fit from chapter 5 (orange line) with change point (vertical dashed line), experimental data (blue and red circles) for low crosstalk monitoring and modeling experiment, and open loop exploratory data (green circles), and open loop reported data using reported downlink path loss (green squares). 


\subsection{Comparison of measured EIRP and USRD Power per PRB}

The power emitted from the UE is reported by the USRD and measured via the VSA. The reported and measured values show very similar qualitative trends, however they deviate in absolute magnitude and variance. In Figure 6.6, results for the median measured values of PUSCH EIRP per PRB are plotted across all experimental configurations as function of calibrated path loss, which is calculated by adding an offset of $78.1 \mathrm{~dB}$, see A for more information, to the testbed attenuator setting. Each quadrant of the figure represents an experiment (monitoring or modeling) and a cross talk condition (high or low). The data is divided in this way to reflect the large difference in variability between low and high crosstalk conditions, which was also observed in Chapter 5. In Figure 6.7, the median PUSCH power per PRB reported by the USRD is plotted for all measurement configurations.

Figures 6.6 and 6.7 show very similar behavior. For the low crosstalk case, the median power per PRB follows a linear trend with slope approximately one until a saturation point between path loss values of 108-113 dB (attenuator settings of 30-35 dB), after which the data follow a linear trend with a slightly negative slope. In the high crosstalk case, the observations have a large variance at every path loss setting and display no saturation, but there is a decrease in variation between the calibrated path loss values of 103-113 dB (attenuator settings of 25-35 dB).

The quantitative differences between measured and UE-reported power can be explored by taking a median of the median values across the following factors: offered load, scheduling type, $P_{0}$, and $\alpha$. The results of this aggregate analysis are summarized in Tables 6.3 and 6.4. Here, an approximate 95th centile confidence interval for the median of the medians is estimated by computing a standard deviation and multiplying by an expansion factor of 2 . First, we observe that the measured EIRP per PRB is consistently lower than the USRD-reported power per PRB by approximately $3-4 \mathrm{dBm}$. Second, note that the introduction of crosstalk increases the emitted power by $5-6 \mathrm{dBm}$ and also increases the variation, as reflected by the confidence interval. For example, at a testbed attenuator value of zero, or a path loss of $78.1 \mathrm{~dB}$, the median of median powers in the monitoring experiment was $-30.3 \mathrm{dBm} \pm 1.1 \mathrm{~dB}$ for low crosstalk, and $-25.3 \mathrm{dBm} \pm 5.3 \mathrm{~dB}$ for high crosstalk. In comparison, the USRD power per PRB for these two cases was $-26.0 \mathrm{dBm} \pm 1.1 \mathrm{~dB}$ and $-21.0 \mathrm{dBm} \pm 5.9 \mathrm{~dB}$, respectively.

The same trends are observed for the 90th centile responses. Figures 6.8 and 6.9, again show that the qualitative trends for the measured EIRP per PRB and the USRD reported power per PRB are qualitatively similar, and Tables 6.5 and 6.6 show the quantitative differences. As expected, the 90 th centile response begins at a higher power $(\approx 2-4 \mathrm{~dB}$ higher) for the same path loss when compared to the median.

While this level of aggregation brings clarity, it can hide some characteristics of the UE's behavior. Specifically, the difference between simultaneous measurements of the VSA and the USRD total power are not apparent in the aggregated results discussed above. It is therefore informative to investigate the data at different levels of aggregation, e.g., the data on a TTI (1 ms) basis. By comparing the instantaneous differences between measured EIRP and USRDreported PUSCH power per TTI, additional characteristics become evident.

To clarify the different levels of potential aggregation, Figure 6.5 displays a diagram of data collection and aggregation at test time. This diagram represents the whole-plot, split-plot relationship along with the interleaved nature of the monitoring and modeling experiment. The data analyzed in chapter 5 and the previous plots in this chapter are the result of concatenating the two $5 \mathrm{~s}$ captures for each whole-plot and split-plot realization and creating a centile distribution similar to a cumulative distribution function (CDF).

Alternatively, we can group the total power transmitted per TTI as measured and as reported as a series of points. In Figure 6.10 a scatter plot of both the monitoring and modeling experiment are shown. A small number of points $(<$ $0.01 \%)$ with anomalously high $(>20 \mathrm{~dB})$ difference between measured and reported values was excluded. The total number of points for the monitoring experiment was 2,819,066 where 3,651 were excluded. The modeling experiment consisted of 5,042,115 points with 83 being excluded. Figure 6.11 shows a histogram of the difference between the 
two, indicating that the difference for the monitoring experiment is a randomly distributed variable with mean $-7.5 \mathrm{~dB}$, and standard deviation of $-1.3 \mathrm{~dB}$, and the modeling experiment shows a very similar distribution with mean $-7.3 \mathrm{~dB}$, and standard deviation of $-1.3 \mathrm{~dB}$.

\subsubsection{High Crosstalk}

As the statistical analysis noted, there is a high variation of the uplink power at any single attenuator setting in the high crosstalk case, a simple side experiment was performed to validate this effect of crosstalk. An additive white Gaussian noise (AWGN) interference signal was generated and injected on the uplink channel using a vector signal generator (VSG). The power of this interference was controlled using a programmable attenuator. The DUT UE was confirmed to be attached and set to send a fixed data rate $(500 \mathrm{kbit} / \mathrm{s})$ in the uplink. The mean power of the DUT UE was monitored, as the power of the AWGN was varied. In the case of very small injected AWGN powers, no change was observed, the DUT UE mean power remained fixed at - $9 \mathrm{dBm}$. At an incident noise power of $-55 \mathrm{dBm}$ at the eNB the UE's mean power increased to $-8 \mathrm{dBm}$. This power was stable for a dwell time of $1 \mathrm{~min}$. The interference power was further increased to $-45 \mathrm{dBm}$ and then returned to $-55 \mathrm{dBm}$. The UE's mean emitted power was now stable at $3 \mathrm{dBm}$. This confirmed a large, hysteretic variation in mean power for introduced AWGN crosstalk which is consistent with the effects observed in this experiment using structured crosstalk.

In summary, the small side experiment described here was successful in replicating the unpredictable behavior observed in the high-cross talk cases of the modeling and monitoring measurement campaigns. That is, the behavior appears to be insensitive to the interfering signal type (AWGN vs. structured LTE). This suggests that if inter-cell interference is prevalent in real-world deployments, additional investigation of its impact may be valuable to the community. 


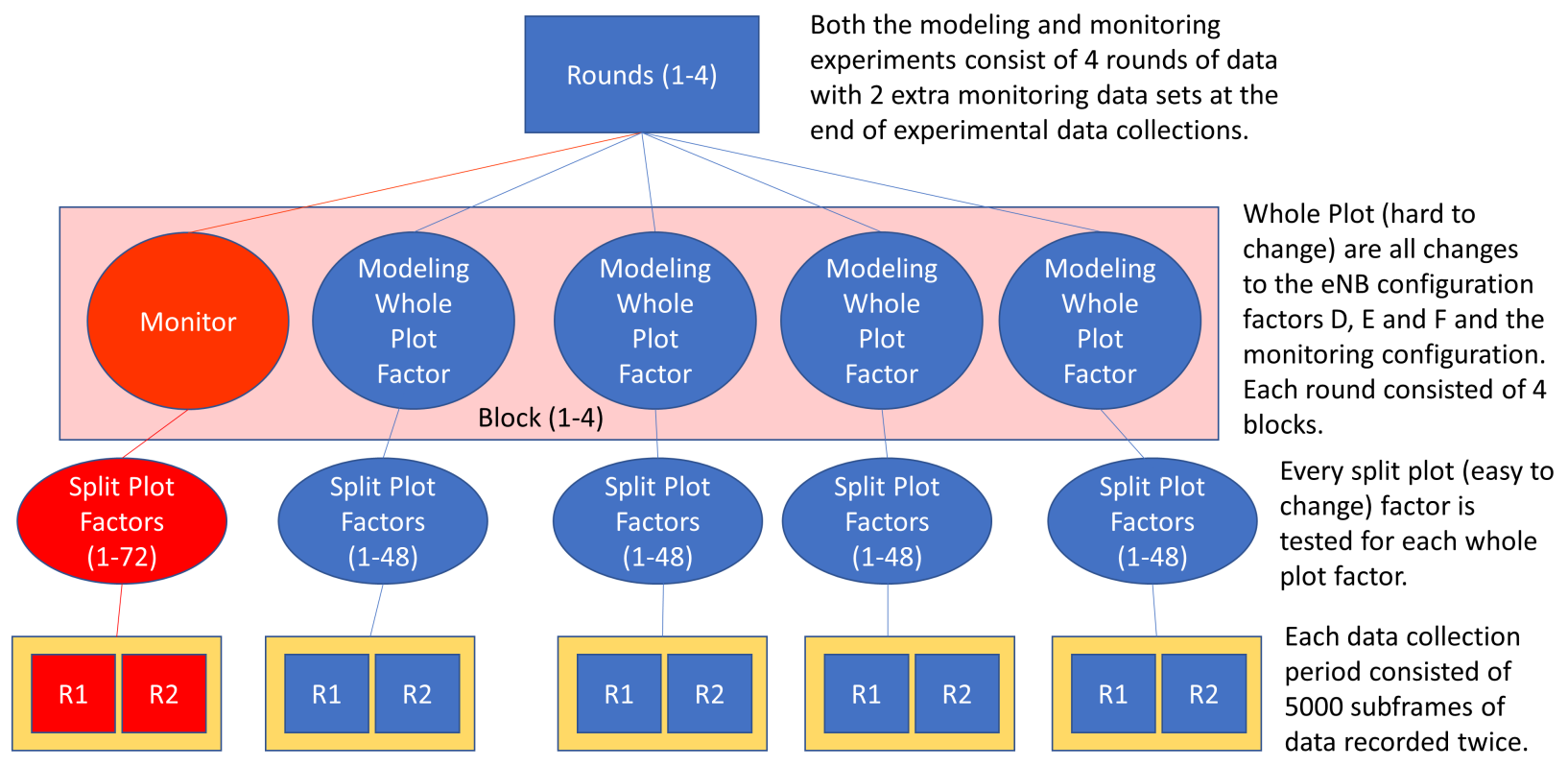

Figure 6.5: Diagram of Data Flow Showing Aggregation Level

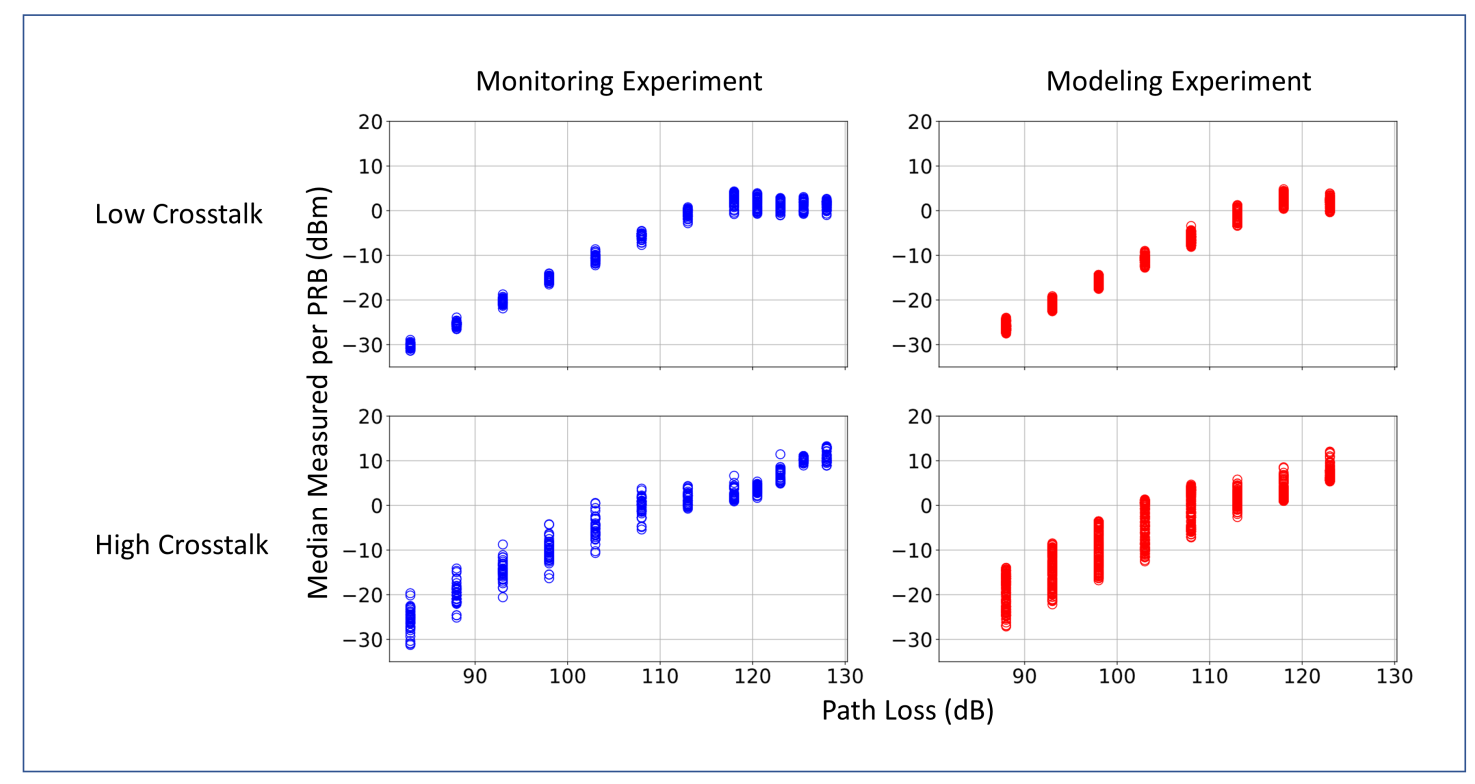

Figure 6.6: Median EIRP per PRB. 


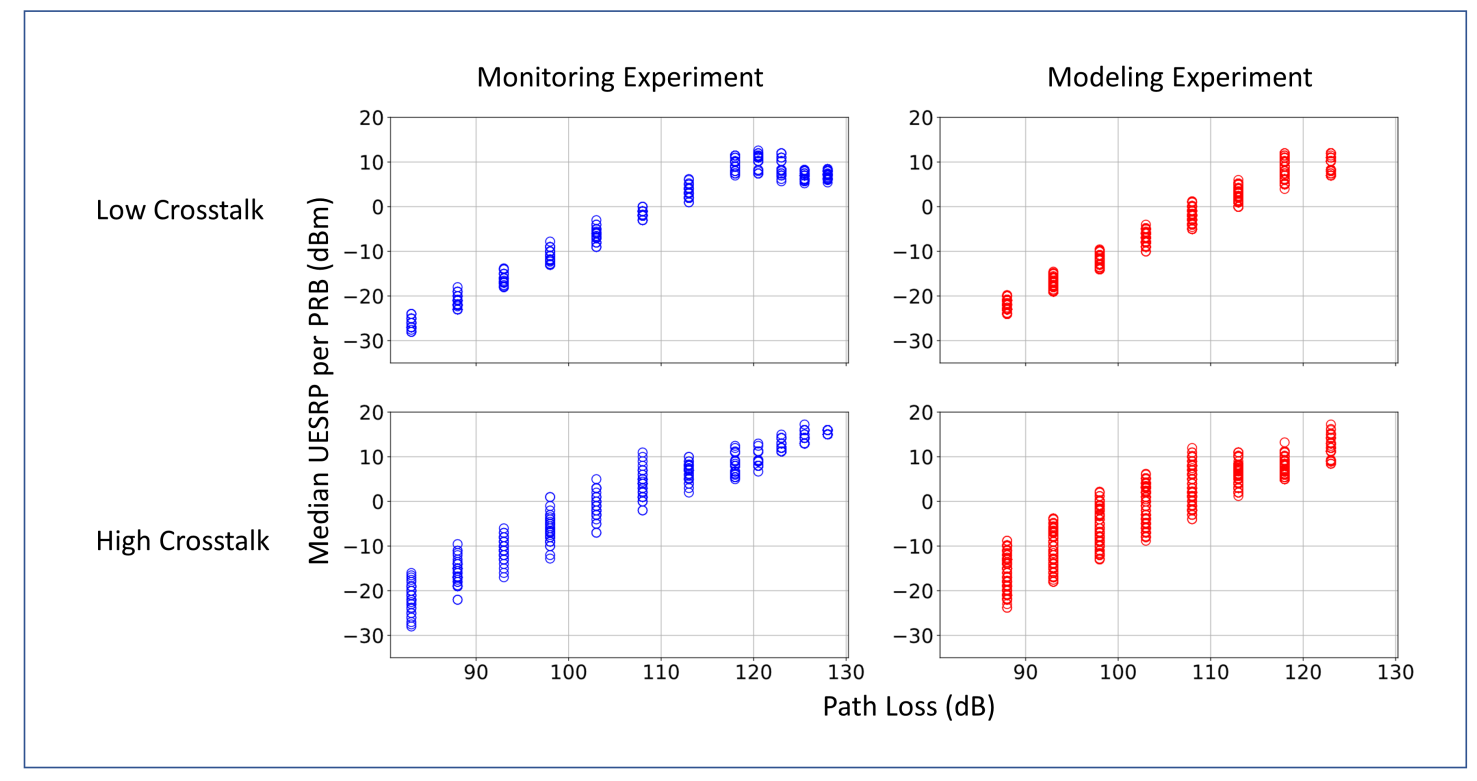

Figure 6.7: Median UE Self-Reported Power (UESRP) per PRB 
Table 6.3: Median of Median Measured EIRP and USRD per PRB for the Monitoring Experiment

\begin{tabular}{lcccccccc}
\hline & \multicolumn{3}{c}{ Measured EIRP $(\mathrm{dBm} / \mathrm{PRB})$} & \multicolumn{4}{c}{ USRD $(\mathrm{dBm} / \mathrm{PRB})$} \\
\hline \multicolumn{4}{c}{ Crosstalk High } & \multicolumn{2}{c}{ Crosstalk Low } & \multicolumn{2}{c}{ Crosstalk High } & \multicolumn{2}{c}{ Crosstalk Low } \\
\hline A & Median & $95 \%$ CI & Median & $95 \%$ CI & Median & $95 \%$ CI & Median & $95 \%$ CI \\
\hline 0.0 & -25.3 & 5.3 & -30.3 & 1.1 & -21.0 & 5.9 & -26.0 & 1.1 \\
5.0 & -20.1 & 4.8 & -25.4 & 1.0 & -16.0 & 5.4 & -22.0 & 1.0 \\
10.0 & -14.7 & 4.3 & -20.3 & 1.1 & -11.0 & 4.7 & -17.0 & 1.1 \\
15.0 & -10.3 & 5.2 & -15.3 & 1.1 & -6.0 & 5.9 & -11.0 & 1.1 \\
20.0 & -5.2 & 5.0 & -10.5 & 1.5 & -1.0 & 5.4 & -6.0 & 1.5 \\
25.0 & 0.2 & 4.2 & -5.6 & 1.4 & 4.0 & 5.9 & -1.9 & 1.4 \\
30.0 & 1.7 & 2.9 & -0.3 & 1.7 & 7.0 & 3.4 & 3.0 & 1.7 \\
35.0 & 1.9 & 2.6 & 3.1 & 2.7 & 8.0 & 4.1 & 9.0 & 2.7 \\
37.5 & 3.7 & 1.6 & 2.0 & 2.8 & 8.9 & 3.0 & 11.0 & 2.8 \\
40.0 & 7.6 & 2.5 & 1.5 & 2.1 & 12.0 & 2.3 & 8.2 & 2.1 \\
42.5 & 10.3 & 1.0 & 1.3 & 2.3 & 14.2 & 2.5 & 7.0 & 2.3 \\
45.0 & 11.1 & 2.9 & 1.5 & 1.8 & 15.0 & 1.0 & 7.2 & 1.8 \\
\hline
\end{tabular}

Table 6.4: Median of Median Measured EIRP and USRD per PRB for the Modeling Experiment

\begin{tabular}{lcccccccc}
\hline & \multicolumn{3}{c}{ Measured EIRP $(\mathrm{dBm} / \mathrm{PRB})$} & \multicolumn{4}{c}{ USRD $(\mathrm{dBm} / \mathrm{PRB})$} \\
\hline \multicolumn{3}{c}{ Crosstalk High } & \multicolumn{2}{c}{ Crosstalk Low } & \multicolumn{2}{c}{ Crosstalk High } & \multicolumn{3}{c}{ Crosstalk Low } \\
\hline A & Median & $95 \%$ CI & Median & $95 \%$ CI & Median & $95 \%$ CI & Median & $95 \%$ CI \\
\hline 5 & -18.3 & 7.3 & -26.0 & 1.6 & -14.0 & 7.6 & -22.0 & 1.6 \\
10 & -13.7 & 7.6 & -21.1 & 1.5 & -10.0 & 7.9 & -17.6 & 1.5 \\
15 & -9.0 & 8.1 & -16.2 & 1.5 & -6.0 & 8.3 & -13.0 & 1.5 \\
20 & -3.9 & 8.5 & -10.7 & 1.6 & -0.0 & 8.2 & -7.0 & 1.6 \\
25 & 0.5 & 7.1 & -6.2 & 1.9 & 5.0 & 8.1 & -2.0 & 1.9 \\
30 & 1.6 & 2.9 & 0.1 & 2.7 & 6.7 & 4.4 & 3.0 & 2.7 \\
35 & 2.1 & 3.1 & 3.2 & 2.1 & 7.0 & 3.4 & 9.0 & 2.1 \\
40 & 6.6 & 2.9 & 2.1 & 2.0 & 11.2 & 3.4 & 10.2 & 2.0 \\
\hline
\end{tabular}




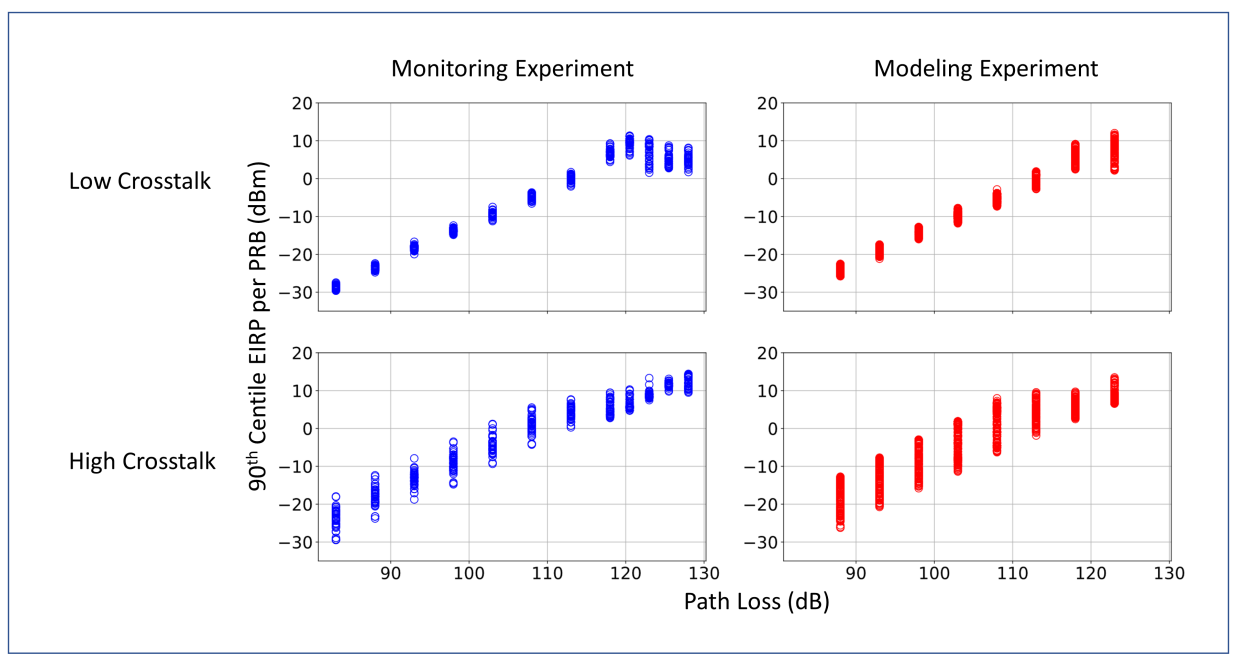

Figure 6.8: 90th Centile EIRP per PRB

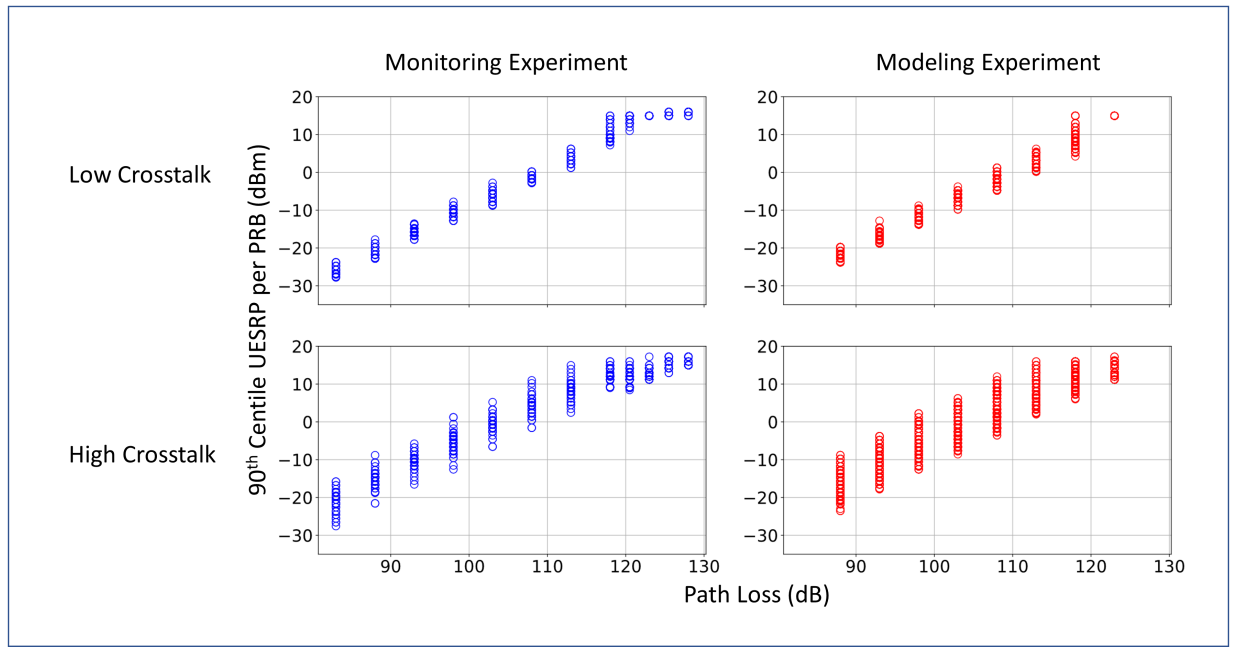

Figure 6.9: 90th Centile UE Self Reported Power (UESRP) per PRB 
Table 6.5: Median of 90th Centile Measured EIRP and USRD per PRB for the Monitoring Experiment

\begin{tabular}{|c|c|c|c|c|c|c|c|c|}
\hline & \multicolumn{4}{|c|}{ Measured EIRP (dBm/PRB) } & \multicolumn{4}{|c|}{ USRD (dBm/PRB) } \\
\hline & \multicolumn{2}{|c|}{ Crosstalk High } & \multicolumn{2}{|c|}{ Crosstalk Low } & \multicolumn{2}{|c|}{ Crosstalk High } & \multicolumn{2}{|c|}{ Crosstalk Low } \\
\hline A & Median & $95 \% \mathrm{CI}$ & Median & $95 \% \mathrm{CI}$ & Median & $95 \% \mathrm{CI}$ & Median & $95 \% \mathrm{CI}$ \\
\hline 0.0 & -23.7 & 5.5 & -28.7 & 1.0 & -20.6 & 5.8 & -25.8 & 2.1 \\
\hline 5.0 & -18.0 & 5.1 & -23.4 & 1.1 & -15.7 & 5.4 & -21.8 & 2.6 \\
\hline 10.0 & -13.0 & 4.1 & -18.2 & 1.2 & -10.0 & 4.6 & -16.7 & 2.4 \\
\hline 15.0 & -9.1 & 5.1 & -13.8 & 1.0 & -5.6 & 5.9 & -10.8 & 2.6 \\
\hline 20.0 & -4.4 & 4.8 & -9.4 & 1.5 & -0.8 & 5.3 & -5.8 & 2.7 \\
\hline 25.0 & 1.2 & 4.9 & -4.8 & 1.4 & 4.2 & 5.7 & -1.8 & 1.6 \\
\hline 30.0 & 4.7 & 3.5 & 0.3 & 1.8 & 9.0 & 5.3 & 3.2 & 2.7 \\
\hline 35.0 & 5.1 & 4.0 & 6.7 & 2.2 & 12.0 & 4.0 & 10.0 & 4.5 \\
\hline 37.5 & 5.9 & 3.3 & 9.5 & 2.6 & 11.2 & 4.1 & 15.0 & 2.1 \\
\hline 40.0 & 8.7 & 2.0 & 8.0 & 5.1 & 12.4 & 2.8 & 15.0 & 0.0 \\
\hline 42.5 & 11.5 & 1.3 & 4.9 & 3.4 & 16.0 & 2.5 & 15.0 & 0.8 \\
\hline 45.0 & 12.0 & 3.2 & 5.3 & 3.1 & 15.0 & 1.9 & 16.0 & 0.8 \\
\hline
\end{tabular}

Table 6.6: Median of 90th Centile Measured EIRP and USRD per PRB for the Modeling Experiment

\begin{tabular}{lcccccccc}
\hline & \multicolumn{3}{c}{ Measured EIRP $(\mathrm{dBm} / \mathrm{PRB})$} & \multicolumn{4}{c}{ USRD $(\mathrm{dBm} / \mathrm{PRB})$} \\
\hline \multicolumn{3}{c}{ Crosstalk High } & \multicolumn{2}{c}{ Crosstalk Low } & \multicolumn{2}{c}{ Crosstalk High } & \multicolumn{2}{c}{ Crosstalk Low } \\
\hline A & Median & $95 \%$ CI & Median & $95 \%$ CI & Median & $95 \%$ CI & Median & $95 \%$ CI \\
\hline 5 & -16.2 & 7.7 & -23.8 & 1.5 & -13.8 & 7.5 & -21.8 & 2.4 \\
10 & -12.4 & 7.5 & -18.8 & 1.6 & -9.6 & 7.9 & -16.8 & 2.4 \\
15 & -7.9 & 7.8 & -14.4 & 1.5 & -5.6 & 8.1 & -12.8 & 2.6 \\
20 & -3.2 & 8.2 & -9.7 & 1.8 & 0.3 & 8.1 & -6.8 & 2.7 \\
25 & 1.5 & 8.3 & -5.3 & 1.9 & 5.2 & 8.1 & -1.8 & 2.8 \\
30 & 4.0 & 5.2 & 0.7 & 2.7 & 9.2 & 7.9 & 3.2 & 2.9 \\
35 & 5.1 & 3.4 & 6.5 & 3.3 & 12.0 & 5.4 & 9.2 & 5.0 \\
40 & 8.5 & 2.9 & 8.8 & 4.6 & 12.0 & 3.2 & 15.0 & 0.0 \\
\hline
\end{tabular}



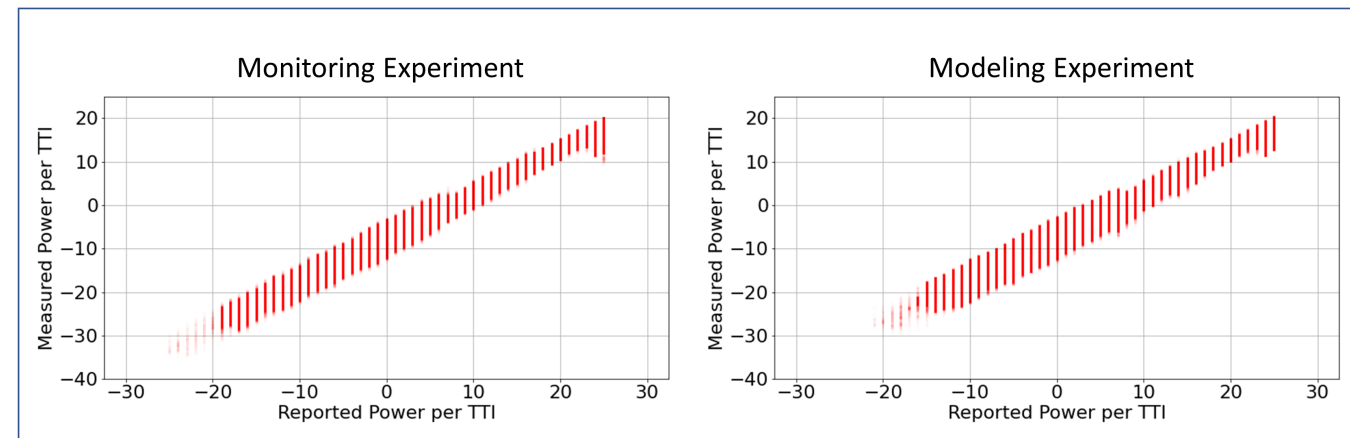

Figure 6.10: Scatter Plot of Measured Versus UE Self-Reported Power (UESRP) per TTI

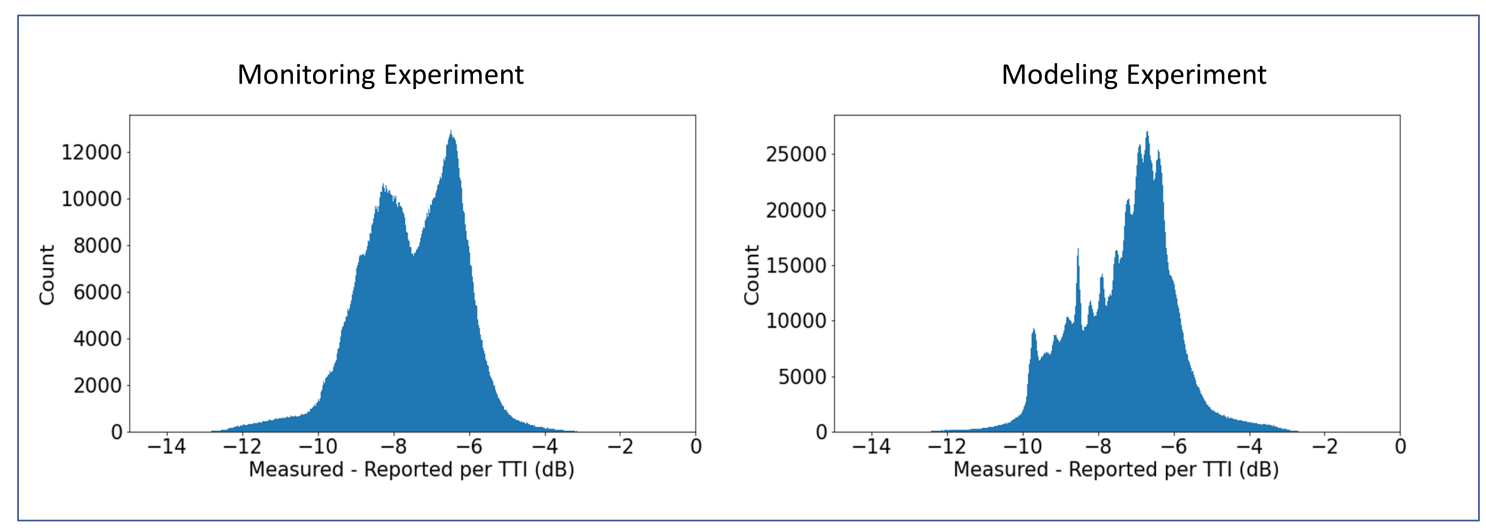

Figure 6.11: Histogram of Measured - Reported Power per TTI 


\subsection{Dynamics of negative power headroom}

A UE is said to be in negative power headroom for a given TTI if the open-loop portion of the LTE power control equation, omitting the MCS-dependent offset, is greater than $P_{\max }$, the maximum transmit power for the UE. When the UE is in negative power headroom, the open-loop component of the power control equation is not, by itself, a useful predictor of UE emission levels. Moreover, it indicates that the UE has been scheduled at a higher data rate than it can support given its current power availability. For these reasons, UE behaviors in negative power headroom conditions are of particular interest to those attempting to predict or model UE emissions.

In this section, we explore the behavior of a UE in a negative power headroom condition. To increase the granularity of the displayed data, we have chosen RSRP (reported in the USRD) to be the x-axis in the Figures 6.13 - 6.17. The RSRP is proportional to the path loss between the UE and eNB in the downlink channel. In this experiment, the reference signal power (RSP) was set to $11 \mathrm{dBm}$. So for a testbed attenuator setting of zero, the RSRP is approximately $-72 \mathrm{dBm}$ and the path loss is approximately $83 \mathrm{~dB}$ (see Table 6.2), this relationship is displayed graphically in Figure 6.12.

The statistical analysis in Chapter 5 revealed that the measured power showed an abrupt behavior change at $33 \mathrm{~dB}$ of added attenuation, which is equivalent to an RSRP-calculated path loss of $115 \mathrm{~dB}$ (RSRP $=-104 \mathrm{dBm}$ ). The RSRP for each value of attenuator setting shows a $95 \%$ confidence interval of $\pm 3 \mathrm{~dB}$ at both $30 \mathrm{~dB}$ and $35 \mathrm{~dB}$ of added attenuation. The RSRP confidence interval was estimated using twice the standard deviation of the mean RSRP values for each $5 \mathrm{~s}$ capture with the desired attentuator setting. This confidence interval furthermore aggregated together all non-path experimental conditions for the monitoring experiment. To illustrate the correspondence to negative power headroom, we have added vertical lines at $-104 \mathrm{dBm} \pm 3 \mathrm{~dB}$ on Figures 6.13-6.17 below.

In Figure 6.13, the relationship between the power per TTI and RSRP is shown for all of the data of both the monitoring and modeling experiment, each point representing an average of a $5 \mathrm{~s} \mathrm{capture.} \mathrm{The} \mathrm{saturation} \mathrm{of} \mathrm{total} \mathrm{transmitted} \mathrm{power}$ is evident in the negative power headroom region (RSRP $<-104 \mathrm{dBm}$ ). This effect is easy to identify in the case of low crosstalk and in Figure 6.14 we separate the data points by crosstalk.

The mean MCS, the mean PRB grant size, and the fraction of re-transmissions all show notable changes when the UE is in negative power headroom. In Figure 6.15 the mean MCS for each $5 \mathrm{~s}$ capture is plotted versus the mean RSRP for the same period, separated by crosstalk. Both the low and high cross talk cases show an abrupt change as the UE enters negative power headroom. For the low crosstalk case there is a decrease in a stable mean MCS of around 22 to values as low as 6 . The high cross talk shows a much larger variance in MCS index for values with RSRPs higher than the transition RSRP of $-104 \mathrm{dBm}$. The mean for a five second capture varying from 2 to 22 . The effect in the negative power headroom case is to decrease consistently and have smaller variation.

In Figure 6.16, the mean PRB grant size is shown versus the RSRP. Again both the low crosstalk and high cross talk cases show an abrupt change in behavior. For the low crosstalk case the mean size of the PRB grant begins to increase. For the high crosstalk case, the mean grant size suddenly increases and then decreases. The behavior of the high crosstalk case is not well understood and needs more experimentation to fully understand.

In Figure 6.17, the fraction of retransmissions is calculated by taking the sum of values of MCS that are 29,30, and 31 an dividing by the number of total transmissions. When plotted versus RSRP we see that the high crosstalk case increases to almost $25 \%$ at RSRP values approaching $-118 \mathrm{dBm}$. In contrast, the low crosstalk case saturates at approximately $9 \%$ for most experimental data in the negative power headroom region.

The engineering analysis confirms the statistical analysis' conclusion that the high crosstalk case requires more investigation to understand fully. In the low crosstalk case before the UE emits maximum power, if the eNB requests a higher power to maintain a specified SNR, this can be easily accommodated. In contrast, if the UE is already emitting maximum power $(23 \mathrm{dBm} \pm 2 \mathrm{~dB}$ ) it cannot increase its signal strength. To preserve the SNR, the eNB can request a 
decrease in MCS index, this in-turn requires a corresponding increase in the number of PRBs assigned to retain the same data rate. This interplay is displayed in figure 6.18 for the low crosstalk case. This increase in the number of PRBs for a fixed power creates a smaller power per PRB, giving rise to the slightly negative slope observed in the statistical analysis after the change point. 


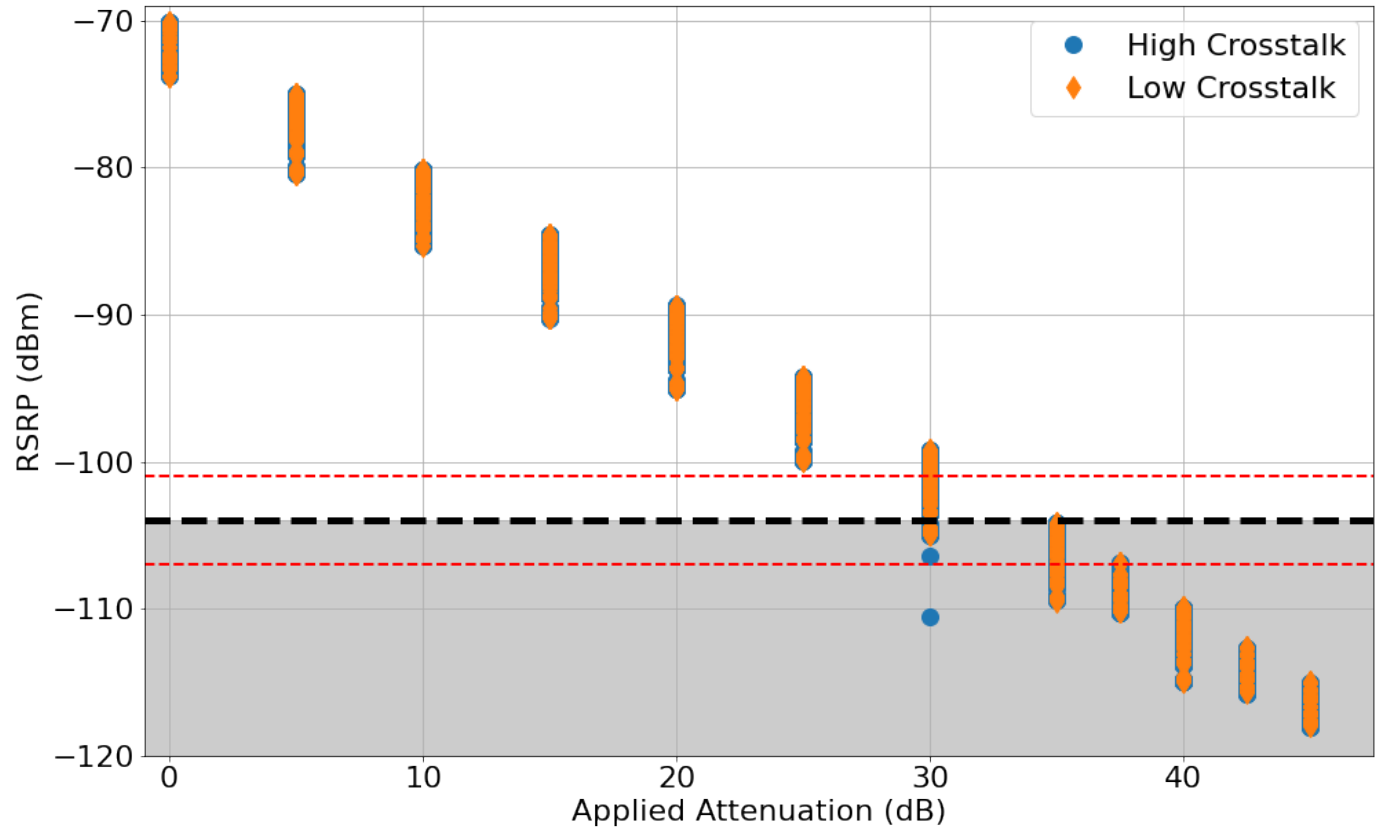

Figure 6.12: Applied Attenuation Versus RSRP. The applied attenuation versus RSRP for high crosstalk (blue circles) and low crosstalk (orange diamonds).The horizontal dashed line represents the change point and the shaded region corresponds with negative power headroom.

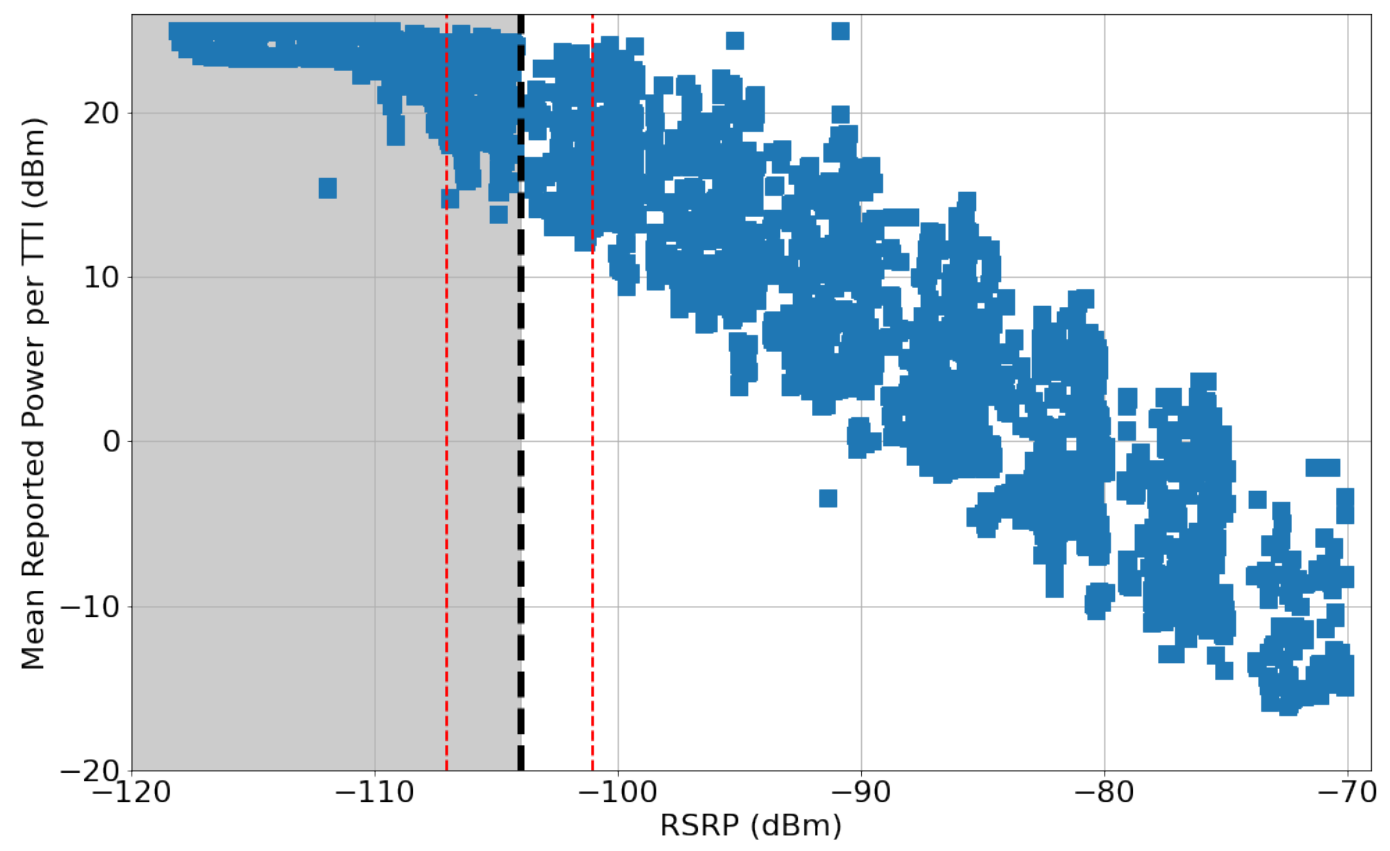

Figure 6.13: Mean Power per TTI Versus RSRP. Negative power headroom is denoted by the shaded region, beginning at the change point (vertical dashed line). 


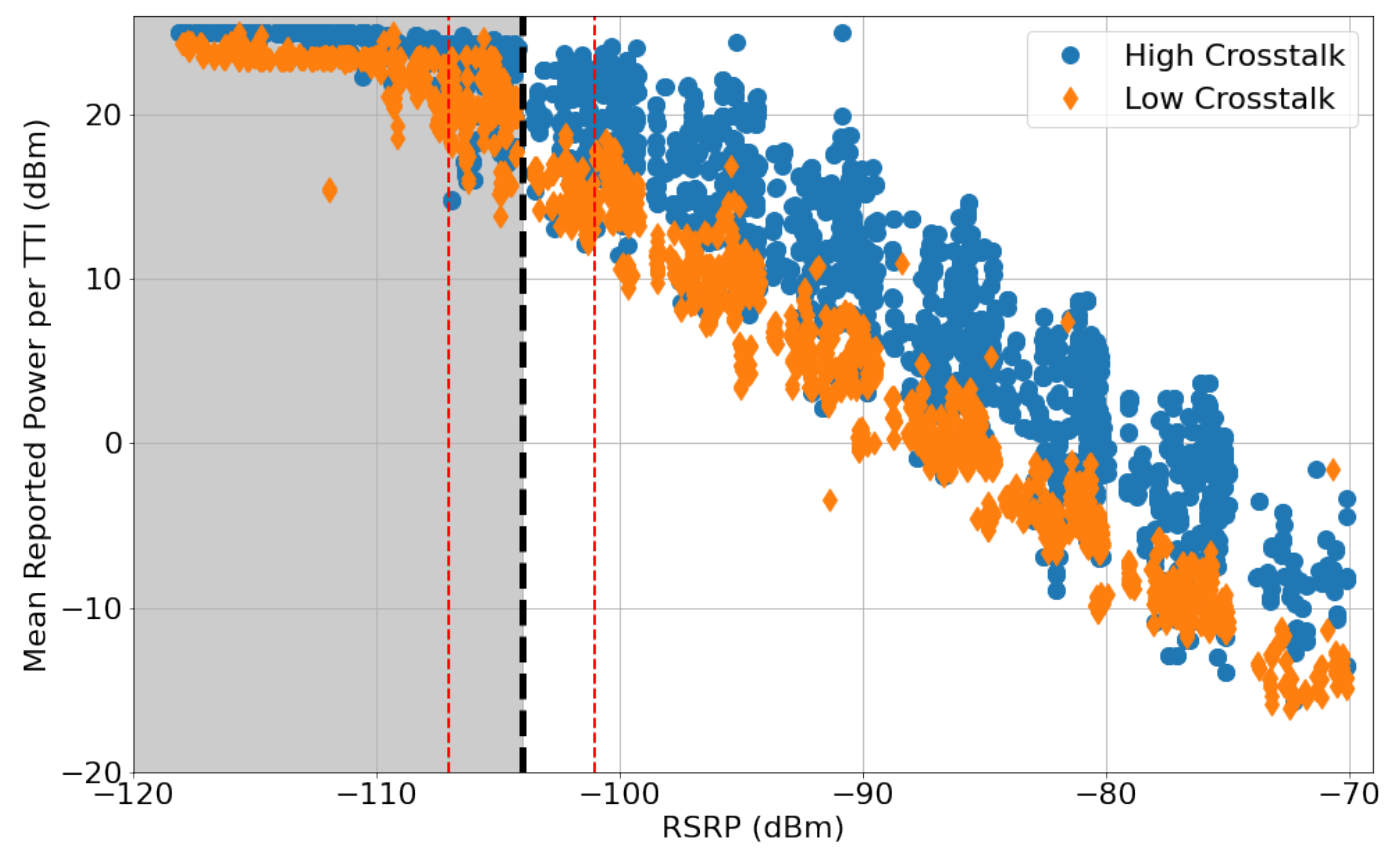

Figure 6.14: Mean Power per TTI Versus RSRP. The mean power from the UE for high crosstalk (blue circles) and low crosstalk (orange diamonds). Negative power headroom is denoted by the shaded region, beginning at the change point (vertical dashed line).

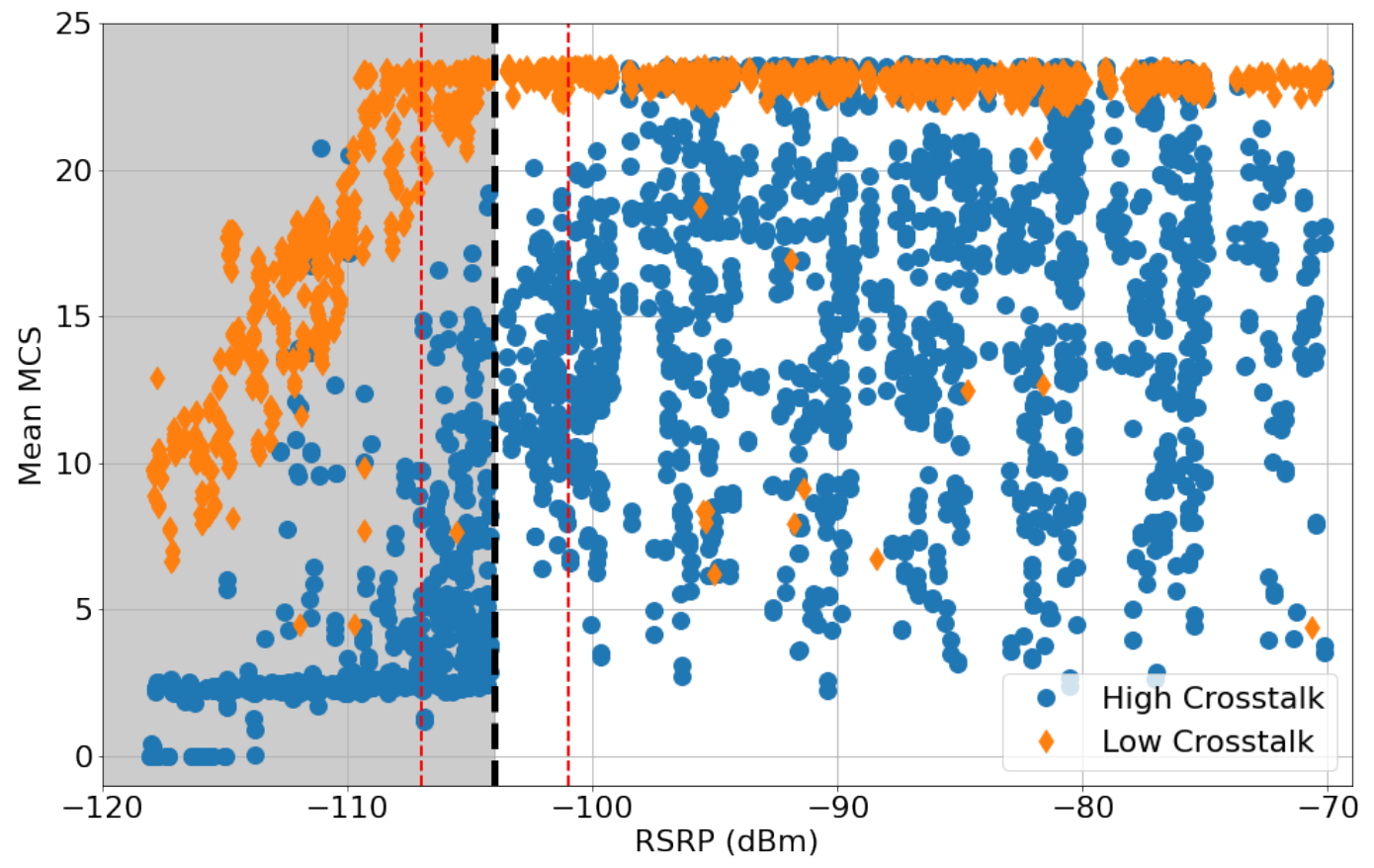

Figure 6.15: Mean MCS per TTI Versus RSR. The mean MCS for each $5 \mathrm{~s}$ capture for high crosstalk (blue circles) and low crosstalk (orange diamonds). Negative power headroom is denoted by the shaded region, beginning at the change point (vertical dashed line). 


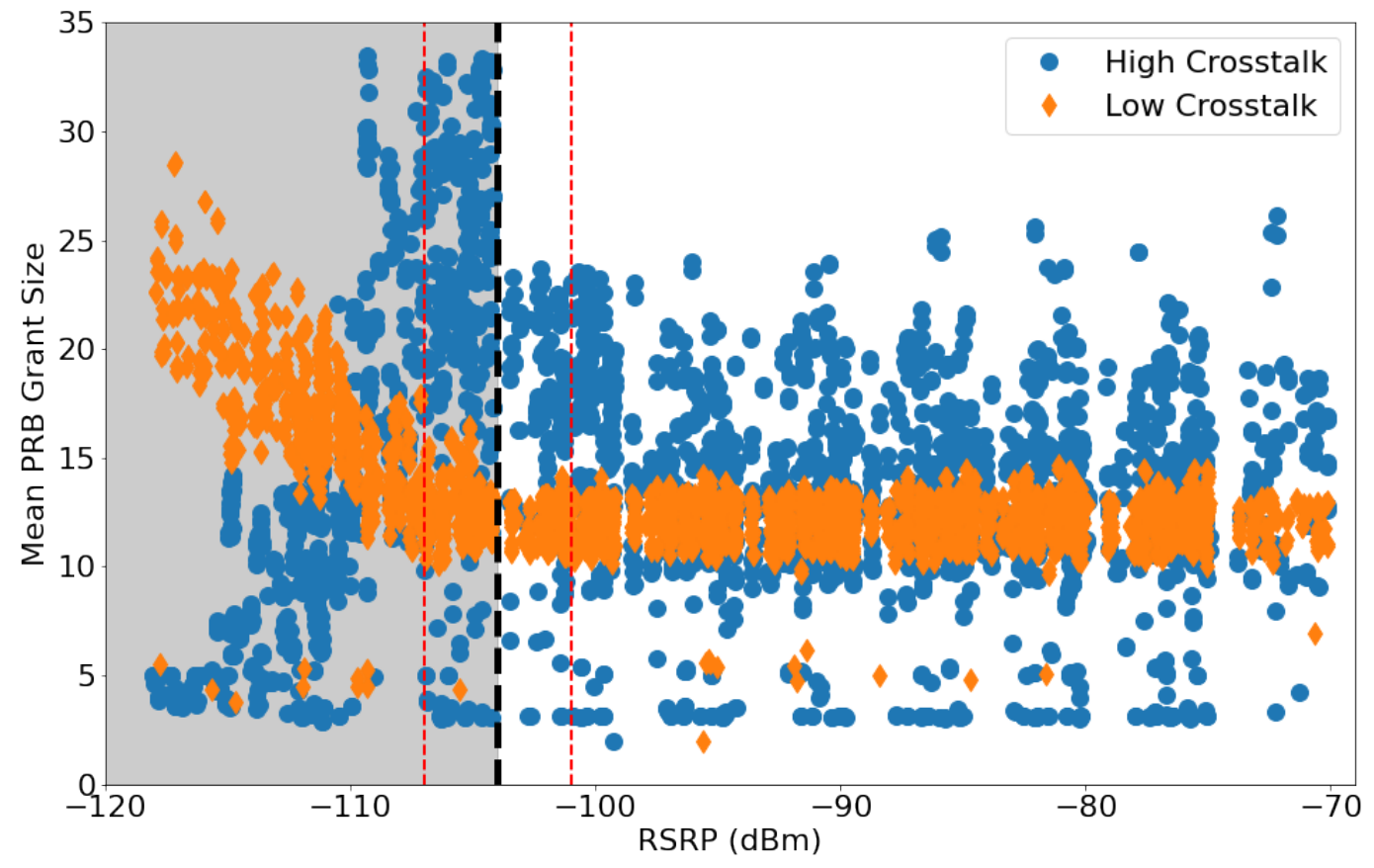

Figure 6.16: Mean PRB Grant Size per TTI Versus RSRP. The mean PRB grant size for each $5 \mathrm{~s}$ capture for high crosstalk (blue circles) and low crosstalk (orange diamonds). Negative power headroom is denoted by the shaded region, beginning at the change point (vertical dashed line).

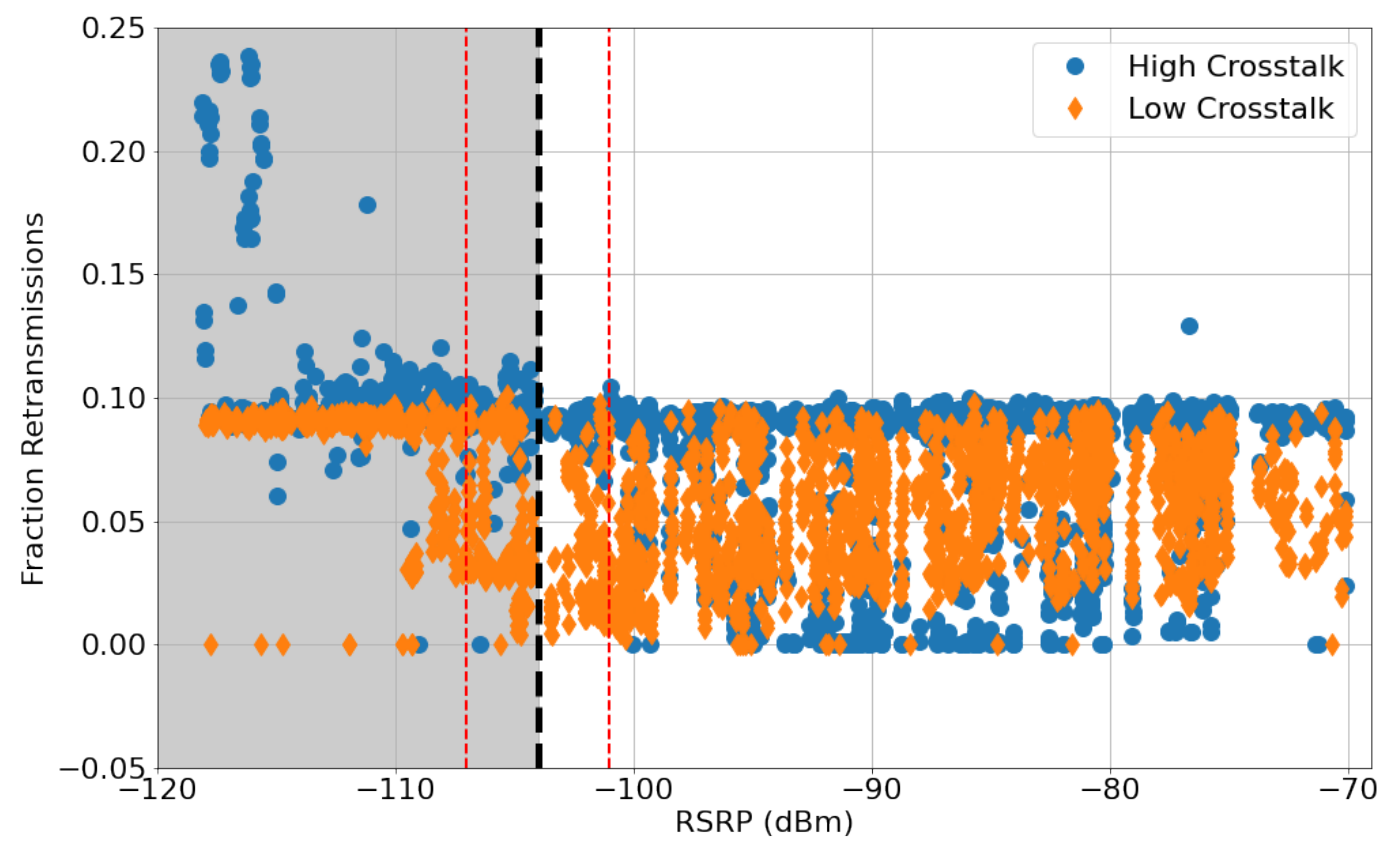

Figure 6.17: Retransmission Fraction Versus RSRP. The number of retransmissions divided by the number of transmissions for high crosstalk (blue circles) and low crosstalk (orange diamonds). Negative power headroom is denoted by the shaded region, beginning at the change point (vertical dashed line). 


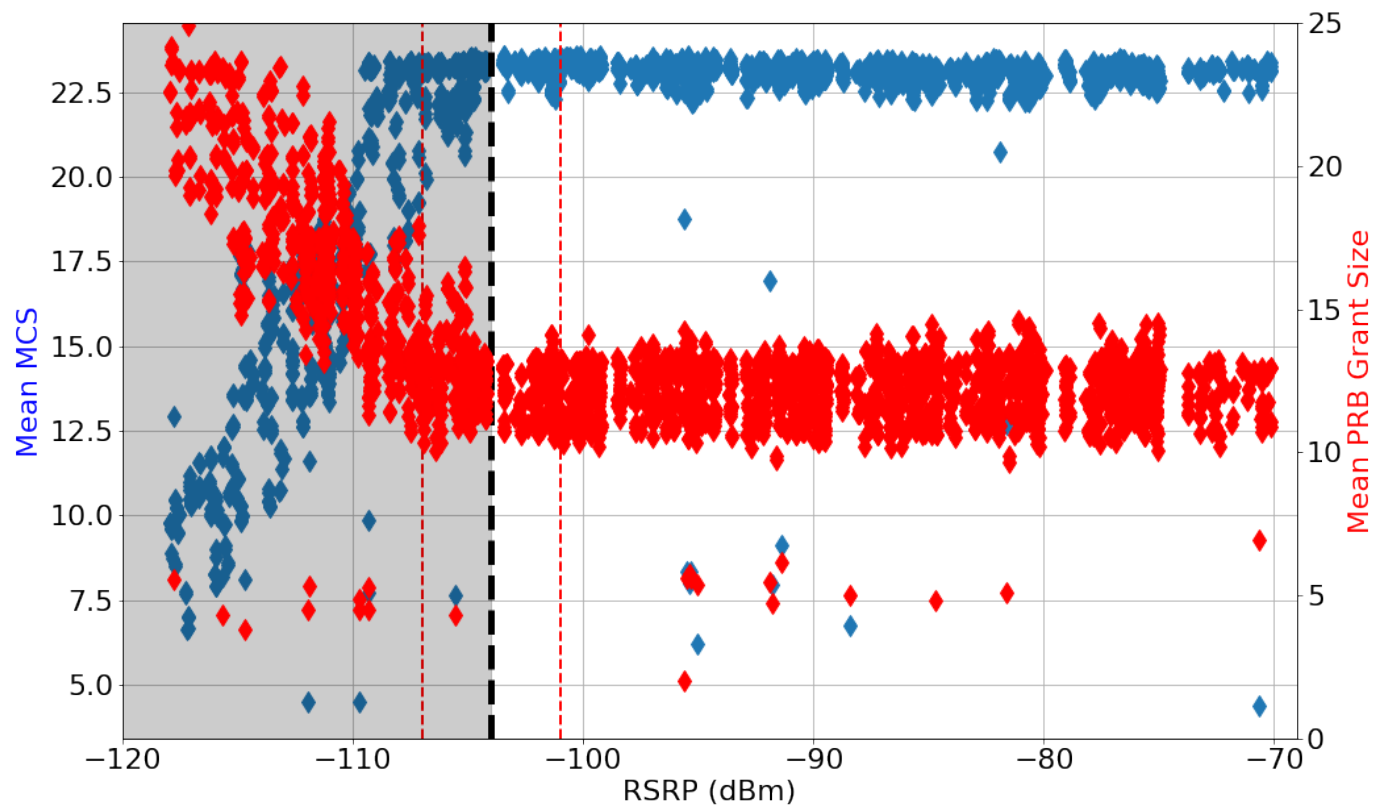

Figure 6.18: Mean MCS index and Mean PRB Size Versus RSRP for Low Crosstalk. The data for the low crosstalk condition showing increasing PRB grant size and decreasing MCS in negative power headroom (shaded region). 


\section{Chapter 7}

\section{Conclusions \& Discussion}

The results of this investigation into UE emissions under close-loop power control are presented in Chapters 5 and 6 on the statistical analysis and engineering analysis, respectively. Below, we summarize the conclusions and takeaways from those analyses. An additional summary of key conclusions from the entire NASCTN project on characterizing LTE UE emissions is provided in Appendix B.

\subsection{Conclusions from statistical analysis}

The closed-loop power control study involved two experiments: a modeling experiment to create descriptive models of measured PUSCH EIRP per PRB and a monitoring experiment conducted in parallel with the modeling experiment for the primary purpose of checking for instability in the testbed. In summary, the main combined statistical findings of these two experiments are:

- Over nearly two months of operation, the testbed was statistically stable.

- Measured PUSCH EIRP per PRB exhibited inexplicably large short-term variation at High crosstalk.

- No statistically discernible handset-contributed variation was present in measured PUSCH EIRP per PRB.

- Continuous, piecewise linear models are proposed to describe the centiles of measured PUSCH EIRP per PRB in the monitoring experiment. The key feature of these models is a changepoint in applied attenuation where the power centile reaches a maximum and then declines.

- The modeling experiment's three ancillary factors $C, D$, and $F$ (offered load, scheduler type, and fractional power control factor) were found to have either no statistically discernible effect or, where an effect was detected, its size is a fraction of $1 \mathrm{~dB}$.

- Statistical quadric response surface models of measured PUSCH EIRP per PRB are proposed to describe its relationship to applied attenuation and eNB nominal power. For applied attenuations below $30 \mathrm{~dB}$ these models take, specifically, the form of a hyperbolic paraboloid. For the full range of collected modeling data, continuous, two-piece, hyperbolic paraboloid models of centile response are speculatively proposed.

\subsection{Conclusions from engineering analysis}

The engineering analysis extended the results of the statistical analysis using other diagnostic information. In summary the main findings are:

- Independent of the underlying settings for $P_{0}$ and $\alpha$, closed loop power control behaves like open loop power control with $P_{0} \approx 108 \mathrm{dBm}$ and $\alpha \approx 1$ for low crosstalk (specific to our testbed). 
- When using UE reported path loss, UE reported power follows the power control equation.

- UE reported path loss often differs substantially (up to $15 \mathrm{~dB}$ ) from calibrated measurements of path loss.

- UE reported power per TTI is on average $7 \mathrm{dBm}$ greater than the measured EIRP.

- Crosstalk increases UE emitted power and its variation.

- In negative power headroom, UE has more scheduled PRBs and a lower MCS index. This leads to a decrease of the power per PRB, reported in the statistical analysis as a slightly negative slope after the change point.

\subsection{Discussion}

\subsubsection{Applications to 5G New Radio}

The NASCTN team acknowledges that this work is done as deployments of 5G New Radio equipment are beginning to roll out. The work done here explicitly targets $4 \mathrm{G}$ networks and devices. We can consider what may be the same or different - as the next generation of technology is deployed.

An examination of the 3GPP specifications reveals only one change to the power control equation for 5G deployments. In $5 \mathrm{G}$, there is an additional term added to the equation that accounts for variable subcarrier spacing. Assuming that the sub-carrier spacing of $5 \mathrm{G}$ deployments in the sub $6 \mathrm{GHz}$ frequency range (known as FR1) is the same as in 4G (15 $\mathrm{KHz}$ ), we expect that the power required to transmit the same number of PRBs by a $5 \mathrm{G}$ UE will be similar to that of a 4G UE. That is, if a $4 \mathrm{G}$ network and UE are measured in one set of environmental, network, and use case conditions, we expect that a $5 \mathrm{G}$ network and UE placed in identical conditions would result in similar power per PRB results. ${ }^{1}$

From the above statement, the big question is: Will $5 G$ network topologies look similar to $4 G$ ? There is a general expectation that $5 \mathrm{G}$ will enable more throughput - in both uplink and downlink. This increased throughput can be achieved with a combination of an addition of new MCS states, an increased use of carrier aggregation, and/or a more dense geographic distribution of cells. A likely consequence of achieving increased throughput will be an increase in the overall PRB utilization (as averaged over a geographic area). Another feature becoming more prevalent with the release of 5G is antenna array beamforming. Designed predominately for use at mmWave frequencies, lower frequencies are also likely to see some beamforming from base stations and possibly from UEs. Beamforming is generally beneficial, as it focuses the emissions in the direction of the base station. The increase in PRB utilization and use of beamforming could impact the over all aggregate emissions of the cell, while still operating within the conclusions and analysis drawn from the NASCTN work.

The transition from $4 \mathrm{G}$ to $5 \mathrm{G}$ network deployments and topologies is likely to be a lengthy transition. For comparison, carriers in the U.S. are still transitioning away from $3 \mathrm{G}$ deployments [18]. During this time, it may be possible to have both 4G and 5G topolgies co-located. It's conceivable that this could also impact the aggregate emissions from a cell while still operating within the results discussed here.

To be certain of the difference 5G networks and UE may cause in the consideration of aggregate emissions, a smaller bridging study could be done to demonstrate if the NASCTN results can be replicated in 5G, or if additional measurements are necessary.

\footnotetext{
${ }^{1}$ These similar per PRB results likely extend into regions of negative power headroom (e.g., indoors, or areas of low RSRP). This extension assumes similar resource scheduling behavior between $4 \mathrm{G}$ and $5 \mathrm{G}$ base stations.
} 


\subsubsection{Future Work}

The work presented here represents a significant contribution to the community's body of knowledge on UE emissions and LTE system behavior. However, open questions remain. Among those questions are the 13 other topics delineated in [1]. Among these topics are more in-depth characterization of: UEs in particular conditions, UE behavior across vendor, UE behavior across eNB vendor, and the influence of the neighboring sectors or technologies (e.g., so called "hand-down" 2 situations). While all 13 topics from [1] remain valid questions, this work does make contributions to many of those topics. Future investigations of should consider these results when designing experiments to explore one of those areas.

In an ideal situation, the community at-large would have a tractable, scientifically rigorous, and statistically sound predictive model with an uncertainty analysis for aggregate emissions from UEs. The NASCTN work does not constitute a predictive model, but it can be used as a step toward a predictive model. To create a predictive model, an additional large-scale measurement campaign would be necessary to collect enough data to make a reliable predictive model and develop an uncertainty analysis. This measurement campaign could leverage the NASCTN results to make informed decisions about where to concentrate these measurements. For example, NASCTN has shown that high-crosstalk situations produce large variations in the measurement results. If predicting UE emissions in scenarios involving influence from other cells or interfering signals is desired, a measurement campaign would need to focus heavily on these cases, and less on cases where there is little variation (e.g, significant positive power headroom under closed-loop power control).

Appendix B of this report contains a summary of the results from this report as well as [1]. The appendix also contains a list of elements a potential predictive model may contain. The appendix text describes how the NASCTN work can - or cannot - help inform aspects of the individual predictive model elements. The NASCTN data do not completely "solve" any one element. Therefore, the text in Appendix B can also provide some useful insight into the design and objective of future work.

\footnotetext{
${ }^{2}$ Hand-down situations are instances when, for example, cell in a higher frequency band hands a UE down to a lower frequency band. This may be done to improve coverage or signal for a UE in a negative power headroom situation.
} 


\section{Appendix A}

\section{Uncertainty Budget}

\section{A.1 Uncertainty}

In this measurement campaign, the principal output of the testbed is the maximum EIRP per PRB from a UE. The complete uncertainty budget for this measurement campaign is shown in Table A.1. Grouping the measured and calculated terms into a single equation helps to identify the sources of uncertainty and the relationship between terms. A summary equation for the EIRP is:

$$
\operatorname{EIRP}[\mathrm{dBm}]=P_{\mathrm{SA}}+C_{\text {Through }}+G_{r}+20 \log _{10}\left(4 \pi d_{\text {setup }} \lambda_{0}\right)+C_{\text {Align }}+C_{\text {position }}
$$

where:

$$
\begin{aligned}
P_{\mathrm{SA}}= & \text { Power measured at the vector signal analyzer in } \mathrm{dBm} ; \\
C_{\mathrm{Through}}= & \text { Coupling between the input of the spectrum analyzer and the output of the receiving antenna in } \mathrm{dB} ; \\
G_{r}= & \text { Gain of the receiving antenna in } \mathrm{dBi} ; \\
\lambda_{0}=c_{0} f= & \text { the wavelength at the center frequency of the measurement band in free space, expressed in meters; } \\
C_{\text {Align }}= & \text { possible misalignment factor (both in terms of pattern and polarization) between the DUT UE and } \\
& \text { the receiving antenna in } \mathrm{dB} ; \\
C_{\text {position }}= & \text { possible position effects in the small shielded chamber in dB. }
\end{aligned}
$$

\section{A.1.1 Vector Signal Analyzer Power Measurement Uncertainty Components}

Based on specifications given by the manufacturer, the standard uncertainty of a power measurement is $0.3 \mathrm{~dB}$. However, the manufacturer also recommends to increase the uncertainty by $0.2 \mathrm{~dB}$ if measurements are taken over a long time period without aligning the system (this was sometimes required for some of the measurements). Therefore, the standard uncertainty of $0.5 \mathrm{~dB}$ for measurements of power is given using the VSA. Since the signal source was a commercial UE, there was additional variability in the received signal. However, this variability is accounted for separately.

\section{A.1.2 Coupled Output Power Calibration Uncertainty Components}

The RF path needed calibrations so any variations in the testbed before the directional coupler should be observable as changes in power detected by the VSA (the directional coupler and the cables from the incident sidearm to the spectrum analyzer and to the transmitting antenna) ${ }^{1}$. Rather than measure the coupler and cables separately (which increases uncertainty), the coupler and cables are calibrated as a system. The uncertainty in these calibrations is given by a spreadsheet provided by the manufacturer of the network analyzer. (Note: the original spreadsheet downloaded from the the manufacturer's website was non-functional, so technical support provided an older version. The spreadsheet used was Revision 3.0.0, with dynamic link library (DLL) Revision 4, 9, 6, 31). The spreadsheet gave a standard uncertainty of the transmission coefficient for the test conditions of less than $0.1 \mathrm{~dB}$.

\footnotetext{
${ }^{1}$ These components can be seen in Figure 3.1
} 


\section{A.1.3 Programmable Attenuators}

The same models of programmable attenuators used in a previous effort [1] were used in this effort. A conservative standard uncertainty of $0.5 \mathrm{~dB}$ is assigned to these attenuators.

\section{A.2 Offset Calibration to DUT EIRP}

An offset calibration is required to convert the power reading in the VSA spectrogram to EIRP at the DUT UE. The offset correction from the re-binned VSA spectrogram to the VSA input connector is $+7.7 \mathrm{~dB}$ (see [1]), and the correction offset from the VSA input connector to the EIRP at the DUT is measured to be $+54.5 \mathrm{~dB}$. Hence, the total offset correction applied to convert the re-binned VSA spectrogram reading to calibrated DUT UE EIRP is $+62.2 \mathrm{~dB}$. The expanded uncertainty of the calibrated measurement result is $\pm 2.3 \mathrm{~dB}$ (see section A.1). 
Table A.1: Sources of uncertainty and uncertainty budget for VSA captures of UE emissions.

\begin{tabular}{|c|c|c|c|c|c|c|c|}
\hline Classification & Factor & $\begin{array}{l}\text { Probability } \\
\text { Distribution }\end{array}$ & $\begin{array}{l}\text { Evaluation } \\
\text { Type }\end{array}$ & $\begin{array}{l}\text { Designator } \\
\text { Designator }\end{array}$ & $\begin{array}{l}\text { Uncertainty } \\
\text { (dB) }\end{array}$ & $\begin{array}{l}\text { Correction for } \\
\text { Distribution }\end{array}$ & $\begin{array}{l}\text { Standard } \\
\text { Uncertainty }\end{array}$ \\
\hline \multirow{6}{*}{$\begin{array}{l}\text { Antenna } \\
\text { Calibration }\end{array}$} & Non-ideal Antennas & Normal & $\mathrm{B}$ & U1 & 0.5 & 1 & 0.5 \\
\hline & Spatial variations & Normal & $\mathrm{A}$ & $\mathrm{U} 2$ & 0.3 & 1 & 0.3 \\
\hline & Network analyzer calibration & Normal & $\mathrm{B}$ & U3 & 0.1 & 1 & 0.1 \\
\hline & Frequency flatness & Normal & A & U4 & 0.3 & 1 & 0.3 \\
\hline & Polarization mismatch & Normal & $\mathrm{B}$ & U5 & 0.1 & 1 & 0.1 \\
\hline & Separation distance & Normal & $\mathrm{B}$ & U6 & 0.5 & 1 & 0.5 \\
\hline \multicolumn{2}{|c|}{ Instrumentation Spectrum an } & Normal & $\mathrm{B}$ & U7 & 0.5 & 1 & 0.5 \\
\hline \multirow{5}{*}{ Testbed } & Calibration & Normal & $\mathrm{B}$ & U8 & 0.1 & 1 & 0.1 \\
\hline & Antenna mismatch & U-shaped & $\mathrm{B}$ & U9 & 0.1 & 0.5 & 0.05 \\
\hline & Antenna connection repeatability & Normal & A & U10 & 0.1 & 1 & 0.1 \\
\hline & Separation distance & Normal & A & U11 & 0.5 & 1 & 0.5 \\
\hline & Chamber uniformity & Normal & A & $\mathrm{U} 12$ & 0.3 & 1 & 0.3 \\
\hline \multirow{2}{*}{ Total } & Combined standard uncertainty & & & & & & 1.1 \\
\hline & Expanded uncertainty $(\mathrm{k}=2)$ & & & & & & 2.3 \\
\hline
\end{tabular}




\section{Appendix B}

\section{Applications to Modeling: A Summary of NASCTN Findings}

\section{B.1 Introduction}

Since the NASCTN project proposed by DSO on characterizing UE emissions began in May 2018, a variety of insights into LTE device and system behavior have been uncovered. These insights are currently distributed across three technical notes: factor screening [1], antenna patterns [19], and the present report on closed-loop power control characterization.

This appendix aims to consolidate insights and make them more accessible to the technical community. These insights are summarized in the context of interference modeling. Although interference modeling was outside the scope of the NASCTN project, viewing contributions in that light may aid future model development efforts.

To make decisions about prospective cellular network deployments near incumbent systems, a predictive model for aggregate UE emissions is desirable. Hence, for UE-based models of aggregate emissions, a predictive model for UE emissions is a necessary component. The development and validation of a predictive model for UE emissions was beyond the scope of this NASCTN project, which focused on descriptive modeling. However, the experimental results presented in this report and in NASCTN's prior technical report [1] can inform future experimental investigations aimed at developing predictive models for UE emissions. The key findings of these two reports are summarized here.

\section{B.2 Descriptive, Explanatory, and Predictive Models}

Three dominant data modeling paradigms are generally recognized: descriptive, explanatory, and predictive. Review of these paradigms helps frame NASCTN's contributions, how these contributions can be used, and their roles in potential future work. These modeling approaches have distinct perspectives, goals, and uses. Hence, a clear understanding of these paradigms facilitates effective experimental design and modeling, which is crucial for scientific progress. These paradigms are presented in detail in the paper by Shmueli [20].

Table B.1 summarizes the differences between descriptive, explanatory, and predictive models. Descriptive modelling aims to summarize measured data in a compact, interpretable manner that captures underlying associations between dependent and independent variables. For example, statistical regression models are generally descriptive in nature.

Table B.1: Characteristics of descriptive, explanatory, and predictive models.

\begin{tabular}{|l|l|l|l|l|}
\hline Modeling Paradigm & Purpose & Key Question & Example & Interpretable \\
\hline Descriptive & Summarize structure in data & What happened? & Regression model & Yes \\
Explanatory & Causal explanation & How did it happen? & Physical law & Yes \\
Predictive & Predict new or future outcomes & What could happen? & Neural network & Maybe Not \\
\hline
\end{tabular}


Qualitative information about a relationship among variables is a frequent aim of descriptive modeling. A descriptive model might seek to capture the presence of an abrupt change point in the relationship between two variables; for example as in fluid models that capture the boundary between laminar and turbulent flow. Or a descriptive model might be used to identify factor settings that robustly optimize a system response. Both these models, of fluid flow and of system optimization, emphasize qualitative insight over prediction. Descriptive modeling is the most commonlyused and developed type of modeling in statistics [20].

By contrast, the goal of explanatory modeling is to test causal hypotheses or explanations, usually arising from theoretical constructs [20]. For example, physical laws derived from theory, such as free-space path loss for RF propagation, can be verified with explanatory modeling. Explanatory models are typically straightforward to interpret, because they explicitly quantify causal relationships between independent and dependent variables.

Predictive modeling aims to apply a statistical model or algorithm to predict the outcome of an unknown event, regardless of when it occurred. An example of a predictive model is a neural network trained to estimate future real estate values. Because predictive models are typically developed for predictive accuracy, they can be "black-box" algorithms that are not interpretable through cause and effect relationships.

One way to gain more insight into the differences between modeling approaches is through the bias-variance decomposition for expected prediction error [20]. Namely, assuming a quadratic loss function, the expected prediction error for a model can be decomposed as [21, p. 223]

$$
\text { Expected Prediction Error }=\text { Irreducible Error }+ \text { Bias }^{2}+\text { Variance. }
$$

The first term reflects the intrinsic uncertainty that cannot be eliminated. The second term is the bias that results from model misspecification. The last term is the estimation variance that reflects variability in the estimated model. Descriptive and explanatory modeling focus on minimizing the bias term to obtain the most accurate representation of the data. On the other hand, predictive modeling tries to minimize both modeling bias and estimation variance, potentially sacrificing descriptive or explanatory accuracy to obtain predictive power.

The above considerations imply that study design and data collection should depend on the modeling approach. Namely, the bias and variance terms may depend on the experimental factors differently, which would lead to different choices about how to best apportion experimental effort. Furthermore, predictive modeling generally requires more data than descriptive or explanatory modeling, since predictive modeling requires independent data sets for fitting a model and assessing its predictive accuracy, whereas descriptive and explanatory modeling use all available data for fitting a model.

When the end-goal is an accurate predictive model, descriptive and explanatory models can inform study designs and where to spend experimental resources. Thus, the descriptive models presented in this report can serve as a starting point for future investigations into predictive models.

\section{B.3 Conceptual Modeling Elements}

Developing an interference model was outside the scope of this work. Interference models and aggregate interference models should consider a variety of factors, many of which have not been addressed by NASCTN efforts. To summarize the NASCTN contributions in terms of contributions to larger modeling efforts, Table B.2 list a variety of factors that could be considered.

In Table B.2, categories of model elements are list across the top. Individual elements are listed below. Note that neither the categories or individual elements are shown in any particular order. Areas where NASCTN work can be leveraged are shaded in green. Details on where the information can be found in NASCTN publications is provided in the subsections below. 


\begin{tabular}{|c|c|c|c|}
\hline User Behavior & UE Hardware & Propagation Channel & Network Behavior/Settings \\
\hline Offered load based on behavior & UE antenna pattern & Propagation model & Cell antenna pattern \\
\hline Application(s) in use & UE orientation & Mobile/Static & eNB scheduling algorithm \\
\hline How often the device is used & UE-to-UE variation & Clutter type & Distribution of UEs in cell \\
\hline & Adherence to power control & Indoor vs. Outdoor & Power control type (open vs. closed) \\
\hline & Performance variation with temperature & Fading type & Adjacent cell interference \\
\hline & Performance variation with age & Multipath (MIMO order) & Misc. eNB setting \\
\hline & Internal algorithms and measurements & & MIMO/Beamforming \\
\hline
\end{tabular}

Table B.2: Categories of potential model elements (bold) and individual elements, listed below categories. 


\section{B.3.1 Elements with NASCTN contributions}

NASCTN contributions may not be sufficient to immediately add to a model, but they can provide meaningful insight. The full details of the test circuit, experiment design, and statistical analysis can be found in the appropriate technical note references.

\section{B.3.1.1 Offered Load/Network Load}

In both the factor screening and closed-loop power control efforts, NASCTN has examined the impact of the offered load, and/or network load on UE uplink emissions. In the factor screening effort, this was represented by changing the data rate of the DUT UE and loading UEs in the measurement configuration. In the closed-loop power control efforts, the offered load was controlled by adding or subtracting loading UEs; all UEs had a constant data rate.

The factor screening results showed that the network traffic level did have a statistically significant impact on the DUT UE's uplink power, but only insofar as the number of UEs present in the serving cell, not in terms of their throughput. The data rate of those UEs was not found to be statistically significant. Contrasting those results with the experiments performed here, we see that the network loading was not found to have a statistically significant impact on the DUT UE's emitted power when closed-loop power control is employed. Viewing the factor screening results in light of this information leads to the conclusion that the impact of network loading on UE emitted power is only experienced in open-loop power control conditions.

\section{B.3.1.2 Application(s) in Use}

During the Factor Screening measurement campaign, the impact of UDP vs. TCP traffic was examined. Though this doesn't cover the entire range of applications, it can provide some insight into the impact.

Although the Transmission Control Protocol(TCP) and User Datagram Protocol(UPD) protocols are at the application layer of the Open Systems Interconnection (OSI) model outside the LTE layers, we examined them to see if a connection-oriented protocol traffic like TCP or connection-less ${ }^{1}$ traffic like UDP have any significant effect on the UE transmission power. The results from factor screening experiment indicated that there is not a statistically significant difference in UE upink emissions between using UDP or TCP traffic types.

Aside from the traffic type, the application(s) in use could also impact the size and frequency of resource block grants. For example, a user conducting a video call would consume an increased number of uplink and downlink resources compared to to a user on a voice call. Other applications may only consume an increased amount of data on the uplink or downlink. During its process, NASCTN did not seek information on the the distribution of applications in use on mobile devices.

\section{B.3.1.3 UE Antenna Pattern}

In conjunction with the NASCTN Factor Screening effort, a parallel effort was made to characterize the threedimensional antenna pattern for a selection of UEs [19]. As part of this measurement effort, antenna patterns covering $4 \pi$ steradians were measured for six different UE models. The UE models were selected such that they spanned a variety of costs, physical sizes, and operating systems. A link to anonymoized antenna pattern data is available in [19].

Analysis of the data also included histograms of the EIRP data. The distribution formed by these histograms may be useful as an input into interference models. For convenience, a summary of EIRP histograms from each UE is shown below. This figure can also be found in [19] as Figure 4.1.

\section{B.3.1.4 UE-to-UE variation}

The NASCTN contributions from factor screening, antenna pattern measurements, and closed-loop power control don't address UE-to-UE variation across the market space. However, all three efforts did attempt to get a snapshot

\footnotetext{
${ }^{1}$ Note that connection-oriented traffic expects to establish a connection between the client and server before sending data and waits for acknowledgment from the client, in contrast to the connection-less protocol where such a connection is not expected before sending data.
} 

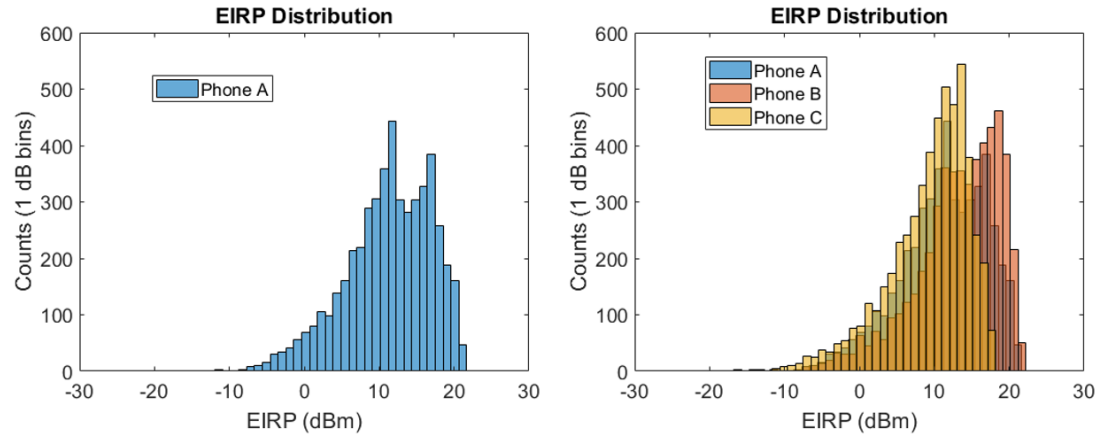

A.

B.

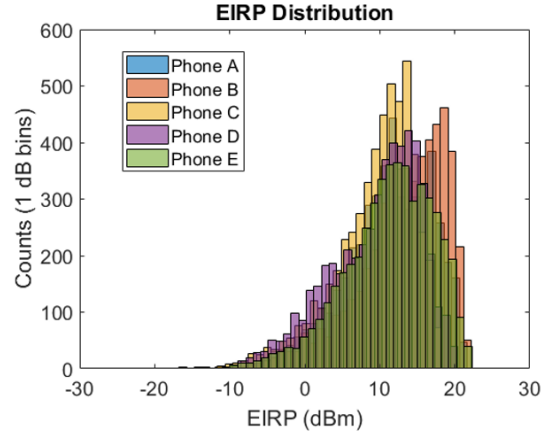

C.

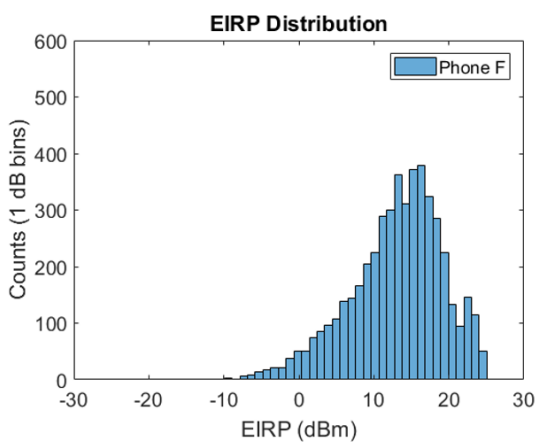

D.

Figure B.1: Histograms of the EIRP measured at all the angles with both horizontal and vertical polarization of the measurement antenna. A.) The histogram of only phone A. B.) Histogram of Phones A-C. C) Histogram of Phones A-E for comparison D.) Histogram for phone F. 
of UE-to-UE variation. The antenna pattern measurements examined the antenna patterns of six UE models. For two models of the UE, two different serial numbers were tested. In the factor screening measurements, two different serial numbers of the same UE model were tested across the measurements. During the closed loop power control experiments, four different serial numbers were examined during the course of the measurement campaign. The four UEs tested during the closed loop power control campaign were intentionally purchased at different times (from the same vendor) in an attempt to get devices that were manufactured at different times.

As discussed in Section 5.2.3, the UE-to-UE (i.e., serial number to serial number) variation was not found to be statistically significant. Similarly, in the UE antenna pattern measurements [19] no significant difference between serial numbers was observed. Note that in the UE antenna pattern measurements, an explicit statistical analysis comparing the difference between serial numbers was not performed. However, based on the measured antenna patterns and histograms, no significant difference was observed. The UE antenna pattern measurements do show a significant difference between UE models, both in terms of EIRP and pattern shape. It is unclear whether this model-to-model difference carries on to other aspects of UE performance.

In each of the three NASCTN measurement efforts, the primary goal was not to quantify the UE-to-UE difference. It would be risky to assume the differences (or lack thereof) seen in the NASCTN measurements are representative of market-wide variation. To better characterize the UE-to-UE variation (both across models and serial numbers) a more targeted experiment involving many more UEs (more models and more serial numbers) is necessary.

\section{B.3.1.5 Adherence to Power Control Equation}

The uplink power control equation 6.1 determines the power the UE attempts to emit, and is based on the number of PRBs assigned, the modulation index, the downlink path loss, and feedback from the eNB. This experiment and the experiment outlined in [1], found multiple sources of discrepancy between the actual measured power and this calculated power. First, the emitted power reported by the UE is not the actual power measured. This effect varies in size, and in this study, for this testbed, was found to be approximately $-7 \pm 1$ for the total power per TTI. In the experiment outlined in [1], the median power per PRB was observed to deviate by a mean of $13 \mathrm{~dB}$ in $16 \%$ of the measurements. In addition, the path loss used in the UE calculation of the power control equation is not the RSP minus the RSRP in log units. It also disagrees with independent assessments of the path loss, This causes the value calculated by equation 6.1 to be significantly different for the path loss used. To create a valid model of UE uplink emissions in open loop power control, the difference between UE self-reported power and measured power and the way the UE calculates the path loss must both be understood. Then the model could predict the UE calculated power and apply a transformation mapping that value to an expected EIRP. In closed loop power control, the eNB plays an important role in calculating the UE power through the use of a transmission control protocol (TCP). This feedback mechanism has a characteristic time, SNR target level, and computation algorithm. To model it effectively, large amounts of data for realistic systems could be used to make an empirical model capable of emulating the EIRP given key parameters of the system.

\section{B.3.1.6 Internal Algorithms and Measurements}

The internal algorithms that determine the UE self-reported path loss and the eNB controlled TCP are unknown and may vary between manufacturers and models of UEs and eNBs. In this experiment, it was observed that these values determine the UE self-reported uplink power, when used in conjunction with equation 6.1 , to the nearest $1 \mathrm{dBm}$. When measured independently, the downlink path loss disagrees with the value used in this calculation. In addition, for a given self-reported power per TTI in this experiment, a measurement of the actual power emitted by the UE randomly differs with a mean of $-7 \mathrm{~dB}$ and a standard deviation of $-1.3 \mathrm{~dB}$. This indicates that the UE reports a power consistent with its calculation of the power control equation, however to an external observer, the emitted power may disagree. 


\section{B.3.1.7 Indoor vs. Outdoor}

Though the NASCTN measurement campaigns didn't specifically attempt to replicate indoor or outdoor scenarios, a range of RSRP values were covered. Other references or measurement campaigns may provide insight into which RSRP values are typical for indoor scenarios. Despite not knowing precisely what range of RSRP values are found in indoor environments, we do know the practical limits for RSRP values in an LTE link. From simple link budget calculations, we can estimate that RSRP values range from -65 to -120 . For outdoor scenarios, the NASCTN factor screening report [1] contains an appendix that describes the distribution of calculated RSRP values for urban, suburban, and rural settings.

\section{B.3.1.8 eNB Scheduling Algorithm}

The factor screening and the closed-loop power control measurement campaigns both examined three different eNB scheduling algorithm types: channel unaware, interference aware, and channel aware. The main difference between these three scheduling algorithms is how the eNB attempts to sense and mitigate the effects of the propagation channel and potential interfering signals. In the factor screening experiment, due to inter-related factor constraints, the scheduling algorithm factor was combined with four other factors, which made interpretation of individual factor effects very challenging. Nonetheless, the analysis showed that as a whole, the factor involving scheduling algorithm type had a statistically-significant impact.

In the closed-loop power control characterization experiment, because fewer factors were considered, factor interdependencies with scheduling algorithm type were not an issue. Consequently, the scheduling algorithm type was a stand-alone factor whose effect could be estimated explicitly. The statistical analysis for the closed-loop power control experiment indicated that the scheduling algorithm type did not have a statistically-significant impact on UE uplink emissions. This finding does not contradict the factor screening results. Rather, it indicates that the scheduling algorithm may have some impact, but only with particular settings for other factors, e.g., open-loop power control.

\section{B.3.1.9 Distribution of UEs in a Cell}

The distribution of UEs in a cell, specifically the loading UEs within a cell was examined during the factor screening measurements, but not the closed-loop power control measurements. During the factor screening measurements, the loading UEs were placed in either an RF "near" condition or an RF "far" condition. Because the factor screening measurements also examined cell radius as a separate factor, there were in effect four different RSRP values (ranging from -50 to $-100 \mathrm{dBm}$ ) for loading UEs in the factor screening measurements. The factor screening statistical analysis was able to separate the impacts caused by cell radius and placement of the loading UEs.

In general, the placement of loading UEs within a cell was not found to have a statistically significant impact on the uplink emissions of a DUT UE. In other words, the fact that other UEs in a cell are placed near the cell edge did not directly result in our DUT UE of interest emitting more uplink power. Note that this statement is focused on the emissions from a single UE. The aggregate emissions from the cell of a whole may be impacted by having many UEs at the cell edge. However, this aggregate impact was outside the scope of the NASCTN measurement campaign.

\section{B.3.1.10 Power Control Type}

The NASCTN data from the factor screening and closed-loop power control reports illustrate some significant differences between the use of open- and closed-loop power control. Figure B.2 from [1], also shown below, quickly illustrates these differences.

The results from the factor screening statistical analysis indicated that the use of closed-loop power control reduced DUT UE uplink emissions by a mean value of $9 \mathrm{dBm}$. Additionally, the CDFs shown in B.2 indicate the range of emissions from closed-loop cases is much narrower than with open-loop cases. From a modeling perspective, creating a descriptive or predictive model of UE emissions in closed-loop conditions is much more feasible than in open-loop conditions. The differences between open and closed loop are large enough, that two different models of UE emissions 

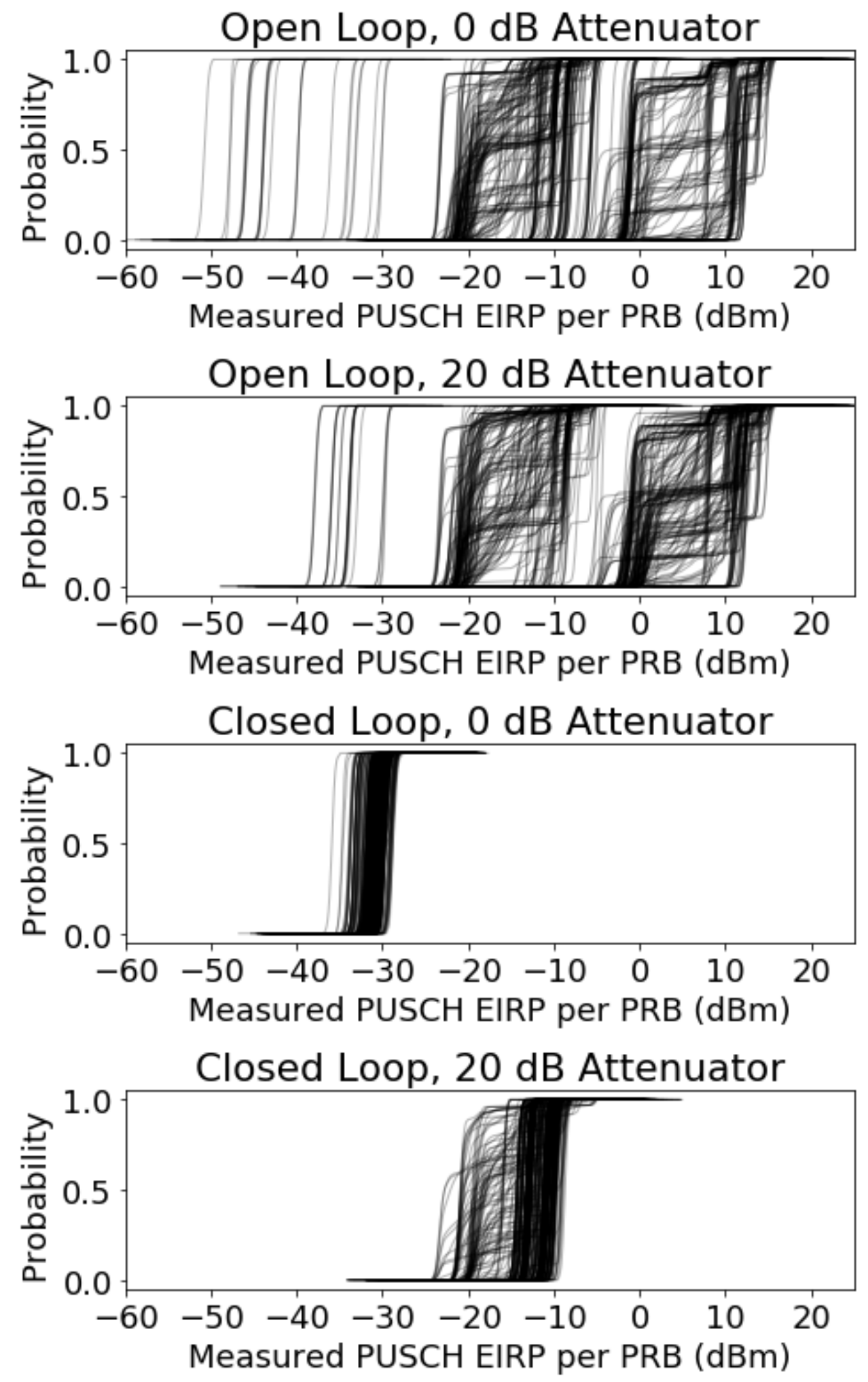

Figure B.2: Summary of empirical CDFs for measured PUSCH power per PRB for all test configurations organized by power control type and testbed attenuation setting. Only data from the factor screening measurements are shown. 
may be necessary - one for each type of power control.

\section{B.3.1.11 Adjacent Cell Interference ("crosstalk")}

In both the factor screening and closed-loop power control measurement campaigns, the impact of cross-talk was analyzed. As outlined in the factor screening report [1], the test circuit ended up being limited in its ability to test crosstalk. The test circuit for the closed-loop power control measurements took a different approach to implementing cross-talk, and indications are that we were successful in implementing crosstalk as desired.

In the closed-loop power control measurements, crosstalk was evaluated at two different levels: "low" (no crosstalk present), and a level that results in a $10 \mathrm{~dB}$ degradation in the SINR as reported by the Cell A eNB. At this $10 \mathrm{~dB}$ level, the amount of crosstalk present in Cell A was enough to cause significant variance in the DUT UE's emissions such that creating a descriptive statistical model was no longer possible given the amount of data acquired in this measurement campaign. The interfering signal imposed on Cell A was the live, structured LTE signal from Cell B. As this may not be a realistic crosstalk signal, an additional measurement was performed with an AWGN signal. Even with the AWGN signal, similar results were observed.

Neither the factor screening or closed-loop power control measurements were designed to fully investigate the effects of crosstalk, inter-cell interference, or interference from another source. However, the results indicate that additional study may be warranted if high crosstalk situations are possible in the deployment scenario. As noted, emissions behavior of the DUT UE in high crosstalk conditions is very difficult to model with NASCTN's existing dataset, and developing a predictive model for emissions in a high crosstalk sceanrio would be even more difficult.

\section{B.3.1.12 Misc. eNB Settings}

During the factor screening measurement campaign, a variety of eNB settings were included in the experiments to examine their impact on the DUT UE's uplink emissions. The subset of these eNB settings found to a statistically significant impact are:

- Maximum Uplink Transmission Power

- Minimum PRB Allocation for Limited UEs

- Initial Maximum Number of PRBs

- Uplink Link Adaptation

- Cell Scheduling Periodicity Request

- Target Uplink Outer scheduling

The impact of the maximum uplink transmission power explicitly sets the maximum power the UE is allowed to transmit; it's easy to see the impact this setting can have as the UE's RSRP decreases. The remaining factors were shown to have an impact, but the reason may not be well understood. Readers are referred to [1] for the full analysis. It's important to note that though the above factors were shown to have an impact, the values used in real-world eNBs may be such that the impact of these individual factors on the UE uplink emissions is minimal.

\section{B.3.2 Elements without NASCTN contributions}

Elements not shaded green in the table are areas where the NASCTN tests cannot provide direct insight. In most of these cases, the element simply falls outside the scope of the original factor screening experiment, UE antenna pattern measurements, or the closed loop power control characterization experiment.

\section{B.3.2.1 How Often the UE is Used}

None of the NASCTN measurement campaigns covered scenarios with time-varying uplink data rates. In all NASCTN measurements, the offered load was constant for the duration of the test configuration. The NASCTN test setup could be leveraged to study this element by programming the DUT UE to intermittently send data, thus simulating the device being used periodically. Similar programming could be done to the loading UEs generated by the UTG. 
The challenge in evaluating the impact of this element is two-fold. First, one must determine the distribution or frequency of UE usage. Second, the acquisition time will likely need to be increased to cover an appropriate number of use/non-use cycles. In the NASCTN measurements, two five-second captures were taken from the VSA. If evaluating the impact of UE use patterns, ten seconds of data may not be enough to determine the statistical significance of use patterns.

\section{B.3.2.2 UE Orientation}

In all NASCTN measurements, the orientation of the DUT UE relative to the eNB was held constant. As discussed in Section B.3.1.3 the DUT UE's antenna pattern isn't isotropic. Therefore, it is reasonable to expect that a UE's orientation relative to the eNB will have some impact on the amount of power it radiates. This orientation factor could be of interest in interference analyses. One could conceive of a scenario where a null in the UE's antenna pattern is oriented toward the eNB, and the peak of the antenna pattern is directed at an asset being protected from interference. In this case, the UE would increase it's radiated power in an attempt to maintain the same $P_{0}$ at the eNB. This increase would also impact he asset in the main beam of the UE's antenna. This scenario is likely very rare in practice, but it does illustrate that some consideration should be given to the UE's orientation if attempting to model or predict emissions.

NASCTN's measurements on the UE's antenna pattern can give some insight into this quetsion; notably the depths of the antenna's nulls. However, still missing in the analysis is the probability distribution associated with UE orientation. In other words, relative to the eNB, what is the probability the UE will be oriented one way or another?

In addition to the UE's orientation, one could also consider its orientation relative to the user. In the NSACTN measurement campaigns, the UE was effectively placed in free-space within the anechoic chamber. A dielectric foam holder was used to hold the DUT UE during testing. In practice, a UE may be in the user's pocket, held up next to their head, or used in their hand. In each of those cases, the users body can have an impact on the direction and magnitude of emitted energy.

To an extent, the UE's orientation relative to the user and the eNB could be accounted for via the RSRP variable. Regardless of the UE's distance from the eNB, if the user is blocking the line-of-sight path between the eNB and UE, the UE will have a lower RSRP value. This fact could then be compared to the NASCTN results, effectively mapping from the change in RSRP to the change in radiated emissions.

\section{B.3.2.3 Performance Variation with Temperature and Age}

The NASCTN test circuit was not equipped to scientifically vary the temperature and thus was not considered in the experiments. All measurements were conducted in a laboratory space equipped with a temperature-controlled HVAC system. All DUT UEs were left plugged into to power via their USB ports which likely stabilized their temperature throughout the measurement runs.

The age and history of use of the DUT UEs was not a factor in the NASCTN measurement campaigns. The impact of age - or hours of use - is unknown. In both measurement campaigns, all DUT UEs were handled carefully to avoid damaging the units during or between measurements. The DUT UEs used in the factor screening measurements had been used in previous, unrelated experiments prior to being examined as part of the factor screening process, whereas the DUT UEs used in the closed-loop power control measurements were purchased new specifically for the closedloop power control measurements. While it was not verified, it is unlikely that the thousands of hours of testing these DUT UEs have undergone have impacted their radiated emissions behavior.

Based on the monitoring experiments run as part of the original factor screening and closed-loop measurement campaigns, we can draw some conclusions about the stability of the DUT UEs during the course of the measurements. In both cases, the DUT UE's variability during the measurements was not found to significantly influence the results. 


\section{B.3.2.4 Propagation Model}

The impact of time-varying, fading channels was not investigated in the NASCTN testing. Instead, a constant channel with uniform frequency response was implemented with a variable attenuator to adjust the path loss between the UE and the eNB. Practical considerations and prioritization of test resources motivated this choice.

Namely, due to the time-varying, stochastic nature of typical fading channel models, incorporating fading channel emulation into our testbed was expected to result in a significant amount of variability in UE emissions. Consequently, much more test time would have been required to characterize variations and obtain repeatable results. Furthermore, due to the large variety of fading channel models available, it is challenging to select a small, representative set of models for testing. For these reasons, and because many other factors were already being considered, we decided to exclude fading channels from the scope of our experiments.

\section{B.3.2.5 Dynamic Mobile Environments and Multi-path}

Designing and testing use-cases in dynamic mobile environments presents multiple challenges, e.g., designing and implementing repeatable test conditions, adequate test time to characterize variations. Issues complicating such tests include the possibility that emissions behavior could show hysteresis, e.g., transitioning from an indoor environment to an outside environment may be different than outside to inside.

For the above reasons, our testing intentionally focused on static environments.

\section{B.3.2.6 Clutter Type}

Similar to propagation models, our testing did not incorporate models for clutter. In cases where clutter models add a fixed amount of path loss, our test results are applicable.

\section{B.3.2.7 Cell Antenna Pattern and MIMO/Beamforming}

The impacts of antenna patterns, beamforming, or MIMO when used on either the DUT UE, loading UEs, or eNB were not examined in either of the NASCTN measurement campaigns. In both measurement campaigns, the DUT UE was explicitly aligned to achieve maximum coupling to the horn antenna within the small anechoic chamber. In all cases, the loading UEs were configured to behave as though they had isotropic antennas within the UTG.

From the perspective of the eNB antenna pattern, the only way to accurately gauge its impacts on UE emissions within a repeatable, laboratory environment would be to replicate a cell in a large anechoic chamber. This would force nearly the entire test circuit into the radiated domain instead of the hybrid radiated/conducted approach selected by NASCTN. An alternative way of considering the impacts of antenna patterns would be to account for them in the overall RSRP value. For example, if a UE were placed a short distance from an eNB, but outside the main lobe of its antenna, it would see a lower RSRP value than it would compared to being placed the same radial distance from the eNB but within the main lobe. In this light, the NASCTN results could be used if details about the eNB's antenna pattern were known. To use the results in this way, one would need to know the depth of the eNB antenna nulls.

Though MIMO functionality was disabled on the eNB, the use of recieve diversity (i.e., a SIMO system) was considered a variable in the factor screening measurements. This factor was not found to have a statistically significant impact on the DUT UE emissions. We were unable to control the use of receive diversity on the DUT UE.

\section{B.3.3 Factors Not Shown to Contribute to UE Emissions}

In closed loop power control, and in the absence of inter-cell interference, the effective data load of the cell appears to have no effect up to a $40 \%$ data loading. In addition, the fractional power control parameter $\alpha$ seems to have no effect in these conditions. The primary factor responsible for the UE's total transmitted power is observed to be the UE to eNB path loss.

The factor screening results revealed that the following factors did not have a statistically significant impact on the 
mean uplink power of the DUT UE. ${ }^{2}$

- eNB Filter coefficients for RSRP measurements

- Scheduling weight for uplink SRS

- Traffic data rate

- Uplink latency timer reaction

- Spatial distribution of UEs in the serving cell

- Number of blanked PUCCH resources

- PUCCH power control method

- Number of loading UEs in adjacent cell

- eNB Receive Antenna Diversity

- Traffic QCI value

- Spatial size of cell (e.g., macro vs. pico)

Readers are encouraged to refer back to the factor screening report [1] to the exact definition of each factor. The definitions of most of these factors are narrow and require some context. For example, based on the above list it would be inappropriate to conclude that LTE small cells pose less interference risk than a macro cell. To make that determination, additional testing and analysis would be required.

\section{B.4 Other Considerations}

In the process of completing the NASCTN work, two other areas were examined that don't necessarily fit into a conceptual model of interference. However, the insights may still be valuable, particularly for those conducting infield measurements.

\section{B.4.1 Scheduler Dynamics in Negative Power Headroom}

For a RF quiet path in closed loop power control, once the UE to eNB path loss exceeds approximately $115 \mathrm{~dB}$ the UE begins radiating a total power equal to the maximum allowable power. If this does not provide a sufficiently high signal to noise ratio at the eNB, the MCS index is decremented. When the MCS index is decremented, a larger number of PRBs must be assigned to the UE to provide the same data throughput. This in effect creates a lower per PRB power emanating from the UE with a broader spectral range.

\section{B.4.2 Measured vs. Reported Transmit Power}

In both the factor screening and closed loop power control experiments UEs showed a significant difference in the power they report and the measured EIRP. This power difference varies in size, but for the closed loop power control experiment was approximately $7 \mathrm{~dB}$ lower EIRP on average with a standard deviation of $2 \mathrm{~dB}$.

\footnotetext{
${ }^{2}$ This list of factors should remain statistically insignificant if again tested under closed loop power conditions.
} 


\section{Bibliography}

[1] J. Coder, A. Wunderlich, M. Frey, P. Blanchard, D. Kuester, A. Kord, M. Lees, A. Sanders, J. Splett, L. Koepke, R. Horansky, D. McGillivray, J. Ladbury, J. Correia, V. Ramaswamy, J. Fevold, S. Lefebre, J. Johnson, J. Carpenter, M. Lofquist, and K. Hartley, "Characterizing LTE User Equipment Emissions: Factor Screening," National Institute of Standards and Tehnology, Tech. Rep. 2069, Sep 2019. [Online]. Available: https://nvlpubs.nist.gov/nistpubs/TechnicalNotes/NIST.TN.2069.pdf

[2] U.S. Government Publishing Office. (2010) Presidential memorandum: Unleashing the wireless broadband revolution. [Online]. Available: https://www.gpo.gov/fdsys/pkg/CFR-2011-title3-vol1/pdf/ CFR-2011-title3-vol1-other-id236.pdf

[3] National Telecommunications and Information Administration. An assessment of the near-term viability of accommodating wireless broadband systems in the $1675-1710 \mathrm{MHz}, 1755-1780 \mathrm{MHz}, 3500-3650 \mathrm{MHz}$, and 4200-4220 MHz, 4380-4400 MHz bands. [Online]. Available: https://www.ntia.doc.gov/files/ntia/publications/ fasttrackevaluation_11152010.pdf

[4] Federal Communications Commission. Auction 97, Advanced wireless services (AWS-3). [Online]. Available: https://www.fcc.gov/auction/97

[5] P. Hale, S. Genco, J. Coder, M. Lofquist, A. Wunderlich, J. Ladbury, A. Kord, J. Cook, T. Hall, and K. Hartley, "Aggregate LTE: Characterizing User Equipment Emissions Metrology Plan," 2017, unpublished. [Online]. Available: https://www.nist.gov/sites/default/files/documents/2017/11/30/aggregate_lte_ test_plan_update_2017.11.28.pdf

[6] K. B. M. Escheikh, H. Jounin, "Performance analysis of novel downlink scheduling algorithm for lte system," in Proc. of the 2014 IEEE International Conference on Advanced Networking Distributed Systems and Applications. IEEE, 2014.

[7] 3rd Generation Partnership Project, "LTE; Evolved Universal Terrestrial Radio Access (E-UTRA); Physical layer procedures (3GPP TS 36.213 Release 14)," 3GPP, Tech. Rep., 2018. [Online]. Available: https://www.etsi.org/deliver/etsi_ts/136200_136299/136213/14.06.00_60/ts_136213v140600p.pdf

[8] 3rd Generation Partnership Project, "Ts 36.101 evolved universal terrestrial radio access (e-utra); user equipment (ue) radio transmission and reception," release 16.

[9] S. M. Kowalski, P. A. Parker, and G. Geoffrey Vining, “Tutorial: Industrial split-plot experiments," Quality Engineering, vol. 19, no. 1, pp. 1-15, 2007.

[10] D. C. Montgomery, Design and Analysis of Experiments, 9th ed. Hoboken, NJ: John Wiley \& Sons, 2017.

[11] C. F. Wu and M. S. Hamada, Experiments: Planning, Analysis, and Optimization, 2nd ed. Hoboken, NJ: John Wiley \& Sons, 2009.

[12] M. Kutner, C. Nachtsheim, J. Neter, and W. Li, Applied Linear Statistical Models, 5th ed. New York: McGrawHill Irwin, 2005. 
[13] R. C. Littell, G. A. Milliken, W. W. Stroup, R. D. Wolfinger, and S. Oliver, SAS for Mixed Models, Second Edition. SAS Publishing, 2006.

[14] G. Milliken and D. Johnson, Analysis of messy data: Volume III: Analysis of covariance. Boca Raton, FL.: Chapman and Hall/CRC, 2002.

[15] SAS Institute Inc., SAS/STAT User's Guide, Cary, NC.

[16] K. Levenberg, "A method for the solution of certain non-linear problems in least squares," Quarterly of Applied Mathematics, vol. 2, no. 2, pp. 164-168, 1944.

[17] D. W. Marquardt, "An algorithm for least-squares estimation of nonlinear parameters," Journal of the Society for Industrial and Applied Mathematics, vol. 11, no. 2, pp. 431-441, 1963.

[18] Georgi Zarkov. When are mobile carriers shutting down 3G networks in the US? [Online]. Available: https://www.phonearena.com/news/when-will-verizon-t-mobile-att-phase-out-3G_id125233

[19] R. Horansky, J. Coder, and J. Ladbury, "LTE Handset Emissions: Radiation Pattern Measurements," National Institute of Standards and Tehnology, Tech. Rep. 2056, Aug 2019. [Online]. Available: https://doi.org/10.6028/NIST.TN.2056

[20] G. Shmueli, “To explain or to predict?” Statistical Science, vol. 25, no. 3, pp. 289-310, 2010.

[21] T. Hastie, R. Tibshirani, and J. Friedman, The Elements of Statistical Learning: Data Mining, Inference, and Prediction, 2nd ed. New York: Springer, 2009. 


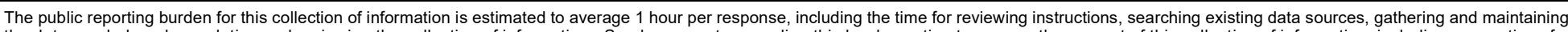

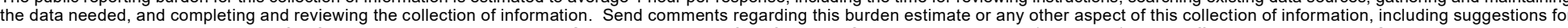

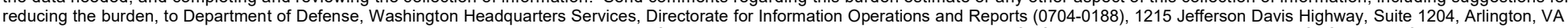

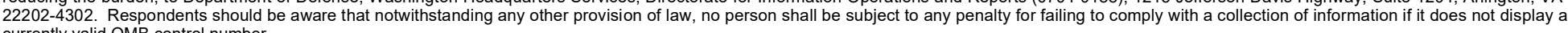
currently valid OMB control number.

PLEASE DO NOT RETURN YOUR FORM TO THE ABOVE ADDRESS.

\begin{tabular}{|c|r|r|}
\hline $\begin{array}{c}\text { 1. REPORT DATE }(D D-M M-Y Y Y Y) \\
05-04-2021\end{array}$ & $\begin{array}{l}\text { 2. REPORT TYPE } \\
\text { NIST Technical Note }\end{array}$ & $\begin{array}{r}\text { DATES COVERED (FrOm - To) } \\
\text { May } 2018 \text { - Arpil } 2021\end{array}$ \\
\hline
\end{tabular}

4. TITLE AND SUBTITLE

Characterizing LTE User Equipment Emissions Under Closed-Loop Power Control

5a. CONTRACT NUMBER

DSO-18-010

5b. GRANT NUMBER

5c. PROGRAM ELEMENT NUMBER

R20-670-0005

5d. PROJECT NUMBER

6700470

5e. TASK NUMBER

000

5f. WORK UNIT NUMBER

8. PERFORMING ORGANIZATION REPORT NUMBER

NIST TN 2147

325 Broadway

Boulder, CO 80305

9. SPONSORING/MONITORING AGENCY NAME(S) AND ADDRESS(ES)

Defense Information Systems Agency (DISA)

ATTN: Defense Spectrum Organization (DSO)

PO Box 549

Fort Meade, MD 20755

12. DISTRIBUTION/AVAILABILITY STATEMENT

Approved for Public Release; Distribution Unlimited.

\section{SUPPLEMENTARY NOTES}

\section{ABSTRACT}

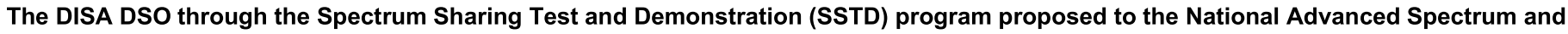

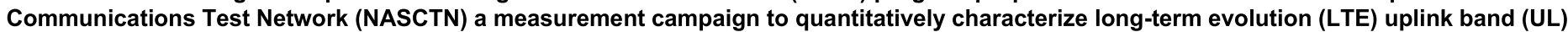

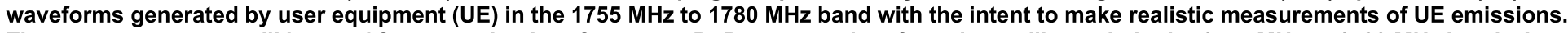

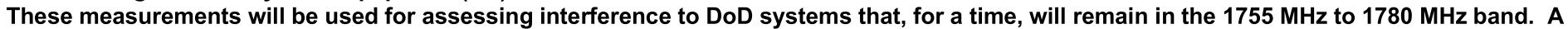

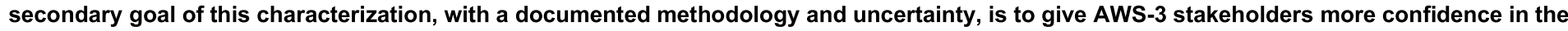
coordination request evaluation (CRE) process.

\section{SUBJECT TERMS}

NASCTN, Closed Loop Power Control, LTE, RF Testing, Metrology.

16. SECURITY CLASSIFICATION OF:

\begin{tabular}{l|l} 
a. REPORT & b. ABSTRACT
\end{tabular}

UNCLASSIFIED UNCLASSIFIED UNCLASSIFIED
17. LIMITATION OF ABSTRACT

None
18. NUMBER

OF

PAGES

83 19a. NAME OF RESPONSIBLE PERSON

Melissa Midzor

19b. TELEPHONE NUMBER (Include area code)

(303) 497-3591 